\title{
Suggested terms and definitions in photocatalysis and radiocatalysis*
}

\author{
N. Serpone ${ }^{\dagger}$ and A. V. Emeline \\ Department of Chemistry and Biochemistry, Concordia University, 1455 deMaisonneuve \\ Blvd. West, Montreal (Quebec), Canada H3G 1 M8
}

\begin{abstract}
This document has two principal goals. First, it summarizes some of the issues that pertain to the complexities encountered in photocatalysis. Secondly, it presents suggested terms and definitions proposed to describe those phenomena, which fall under the umbrella of "photocatalysis" and "radiocatalysis". A distinction is made between the different phenomena that are related to either or both photochemistry and photocatalysis, and to radiation chemistry and radiocatalysis. Consistent definitions of terms in these areas are given. Definitions of important parameters that describe photocatalytic and radiocatalytic phenomena quantitatively are also proposed. As with the related Glossary of Terms in Photochemistry, this document is a dynamic working document to provide the base for lively discussion and debate by the interested community. The terms and definitions will no doubt need to be revisited from time to time as the fields of photocatalysis and radiocatalysis progress and new discoveries reported. Some of the terms used in photochemistry are also given for completion, either verbatim or modified to address the issues of photocatalysis.
\end{abstract}

NOTE TO THE READER: This article of Suggested Terms and Definitions in Photocatalysis and Radiocatalysis is being made available to a worldwide readership through several Journals and Newsletters of photochemistry. The intent of the authors is to provide a base for debate and discussion. The authors welcome comments from interested readers who have constructive suggestions on improving the definitions and perhaps add other terms that will improve and further our overall understanding of a very complex field of chemistry.

\section{INTRODUCTION}

Photocatalysis and related phenomena are now well known and well recognized. Biogenic photocatalytic phenomena, such as those occurring in natural photosynthesis, have been known since prehistoric times and this without any knowledge of the intrinsic chemical mechanisms of plant growth. Abiogenic photocatalytic phenomena were recognized since the initial studies on photochemical phenomena. The term "photocatalysis" was introduced as early as the 1930s, if not sooner. Since then, this term has been used often in the scientific literature. The early workers saw no need to address the nomenclature until the field had

\footnotetext{
* Over the years many people (too many to list here) have contributed to photocatalysis and radiocatalysis. The authors are grateful for the many verbal and written suggestions and comments.

†E-mail: serpone@vax2.concordia.ca
}

matured enough. Nonetheless, the term was taken to represent that field of chemistry that focused on catalytic reactions taking place under the action of light. Consequently, the totality of the phenomena related to both photochemistry and catalysis was considered to belong to the field of photocatalysis. Such phenomena seemed rather exotic and of interest only to a narrow group of specialists.

Recent interest and studies in environmental photochemistry, in natural photosynthesis [1], and in chemical methods for solar energy transformations has contributed greatly to our knowledge and understanding of the various phenomena related to both photochemistry and catalysis. Many of these phenomena differ qualitatively by their nature and, generally speaking, are often found in different fields of chemistry. For example, it is possible to distinguish such phenomena as catalyzed photochemical reactions, photoactivation of catalysts, and photoactivated catalytic processes, among several others. These have led to the necessity to develop a glossary of terms and definitions based on an analysis of the general terminologies associated with both photochemistry and catalysis [2-6].

The glossary proposed herein relies to some extent on the earlier work as a starting point. It does not claim to be exhaustive. Nonetheless, it should be useful to generate and unify the language of communication between the representatives of various fields of chemistry concerned with photocatalysis and radiocatalysis. Of some particular interest are the phenomena connected with solid/liquid and solid/gas heterogeneous systems 
as these have been investigated the most. Where appropriate, however, the terms and definitions will be such that they will also apply to other systems and phases. In this regard, we have borrowed some of the terms used in photochemistry as they are germane to the issues addressed herein.

Some attempts were made in the past to define the term "photocatalysis". Indeed, one of the IUPAC Commissions defined photocatalysis as "a catalytic reaction involving light absorption by a catalyst or a substrate" $[5,6]$. In a later revised glossary [6] a complementary definition of a photo-assisted catalysis was also proposed: "catalytic reaction involving production of a catalyst by absorption of light". Germane to the present discussion, an earlier Compendium of Chemical Terminology [7] described the notion of catalysis and a catalyst as follows: "Catalysis is the action of a catalyst"; and a "Catalyst is a substance that increases the rate of reaction without modifying the overall standard Gibbs energy change in the reaction". Both of these descriptions are less than satisfactory as they lack some, if not many, of the details of the catalytic process. Addition of the extra reactant, namely light, inserts an extra dimension to an otherwise complex issue. An overview of some approaches in describing photocatalytic phenomena was given in a recent paper [8]. It is germane in this context to revisit this overview to attain a better appreciation and understanding of the complexity(ies) of photocatalysis, particularly heterogeneous photocatalysis.

In this context, understanding heterogeneous photocatalysis has necessitated a suitable description of (i) what photocatalysis is, (ii) what turnover quantities are (numbers, TON; rates, TOR; frequencies, TOF) and (iii) how quantum yields $\Phi$ can be established for a seemingly very complex heterogeneous system. For the latter, a protocol for determining $\Phi$ of a photocatalytic process has been proposed [8] and experimental details given [9]. Relative photonic efficiencies, $\xi_{\mathrm{r}}$, also described by Serpone and Salinaro [8] and by Salinaro and coworkers [9] provide a method by which the work from many laboratories in environmental photochemistry can be calibrated when the more auspicious parameter $\Phi$ cannot be assessed because of certain experimental limitations (e.g., immobilized photocatalyst or reactor geometry).

The term photocatalysis has been characterized in the past by a continued use of labels to describe a variety of mechanistic possibilities for a given process. The turnover quantities, while being relatively understood in homogeneous photocatalysis, are not simple parameters to estimate and thus have required further considerations in heterogeneous photocatalysis as they necessitate knowledge of the number of photocatalytically active sites for TON and TOR [10]. These turnover quantities depend on how the photo- catalytic process is described. It is relevant therefore to re-examine briefly the description of photocatalysis as it impacts on some of the major terms and definitions of this glossary.

\section{ISSUES IN PHOTOCATALYSIS}

Unlike the earlier terminology (see above and ref. [7]), it is relevant to recall that catalysis refers simply to a process in which a substance (the catalyst, Cat) accelerates, through intimate interaction(s) with the reactant(s) and concomitantly providing a lower energy pathway, an otherwise thermodynamically favored but kinetically slow reaction with the catalyst fully regenerated quantitatively at the conclusion of the catalytic cycle. When photons are also involved, the expression photocatalysis can be used to describe, without the implication of any specific mechanism, as the acceleration of a photoreaction by the presence of a catalyst. The catalyst may accelerate the photoreaction by interacting with the substrate(s) either in its ground state or in its excited state or with the primary product (of the catalyst), depending on the mechanism of the photoreaction [8]. Note that the latter description is silent as to whether photons also interact with the catalyst. Such a description also embraces photosensitization [11] and yet such a process, defined officially [12] as a process whereby a photochemical change occurs in one molecular entity as a result of initial photon absorption by another molecular species, known as the photosensitizer, is not necessarily catalytic without assessing a turnover quantity and/or the quantum yield. The issue rests entirely on the role of the photons. Chanon and Chanon [13] suggested that the non-descriptive term photocatalysis be taken simply as a general label to indicate that light and a substance (the catalyst or initiator) are necessary entities to influence a reaction. $\left({ }^{1}\right)$ Such a broad description indicates the required reagents without undue constraints as to the (often unknown) mechanistic details of the chemical process (eq. (1)).

$$
\text { Substrate }+ \text { light }+ \text { Catalyst } \longrightarrow \text { Products }+ \text { Catalyst }
$$

${ }^{1}$ ) This cannot be a complete description of photocatalysis. An example of a photocatalytic process of a different type is the photoexcitation of surface metal-oxo complexes on some inert support, e.g., $\mathrm{M}^{2+}-\mathrm{O}^{2-}$. For example,

$$
\begin{gathered}
\mathrm{M}^{2+}-\mathrm{O}^{2-}+h v \longrightarrow \mathrm{M}^{+}-\mathrm{O}^{-} \\
\mathrm{M}^{+}-\mathrm{O}^{-}+(\mathrm{A})(\mathrm{D}) \longrightarrow \mathrm{M}^{+}(\mathrm{A})-\mathrm{O}^{-}(\mathrm{D}),
\end{gathered}
$$

where photoexcitation of the surface metal-oxo complex leads to photoinduced electron transfer from the oxide to the metal to form the reactive state $\mathrm{M}^{+}-\mathrm{O}^{-}$. This reacts with (acceptor)(donor) molecules (A) (D) with the electron localized on the 
Teichner and Formenti [14] described heterogeneous photocatalysis as an increase in the rate of a thermodynamically allowed $(\Delta G<0)$ reaction in the presence of an irradiated solid with the increase (in rate) originating from the creation of some new reaction pathways involving photocreated species and a decrease of the activation energy. In this sense, one could argue that many of the reactions involving irradiated semiconductor photocatalysts belong to the class of photogenerated catalysis (see below). These workers [14] labelled reaction (2) as a photocatalytic oxidation.

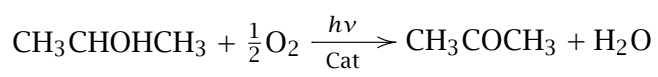

It was also suggested that when $\Phi>1$ (as in reactions involving radical species) the process is catalytic in photons, but when $\Phi \leq 1$ the process is non-catalytic in photons. The latter suggestion was somewhat artificial to the extent that if $\Phi$ were greater than unity, then either the process is a photoinduced catalysis (see below) or the process is a photoinitiated chain reaction, which is not necessarily catalytic. Typically, the quantum yield of a primary reaction step is less than 1 .

In the early 1980s, Salomon [15] proposed that the broad description of photocatalysis be subdivided into two main classes: (i) photogenerated catalysis, which is catalytic in photons, and (ii) catalyzed photolysis, which is non-catalytic in photons (Table 1). In photogenerated catalysis, ground states of the catalyst and of the substrate are involved in the thermodynamically spontaneous (exoergic) catalytic step. By contrast, in catalyzed photolysis either the nominal catalyst T (Figure 1) or the substrate or both are in an excited state during the catalytic step (note: $\mathrm{C}$ is the catalytic entity). Kutal [16, 17] clarified Salomon's formal schemes and Hennig and coworkers [18] proposed consistent labels that applied to experimental observations. A quagmire of mechanism-specific labels appeared subsequently (see Chanon and Chanon [13] for an elaborate account). This called attention to usage of the more broad description of photocatalysis as suggested by Chanon and Chanon [13] and later emphasized by Serpone and coworkers [8, 9, 19] (see above). Figure 1(a) illustrates a simple scheme of photogenerated catalysis, whereas Figure 1(b) depicts an example of catalyzed photolysis.

The distinction between an assisted photoreaction $($ TON $<1)$ and a catalyzed photoreaction $($ TON $\gg 1)$ seems to pose no difficulty in homogeneous photocatalysis since turnover numbers can be described. This is not the case in heterogeneous photocatalysis where a solid catalyst semiconductor, for example $\mathrm{TiO}_{2}$, fulfills

metal cation or on the hole-like state $\mathrm{O}^{-}$. Note that there is no generation of free electrons and holes here. This is local excitation. A similar process is described by mechanism I (see below), which is also an example of local photoexcitation without photogeneration of free electrons and holes.

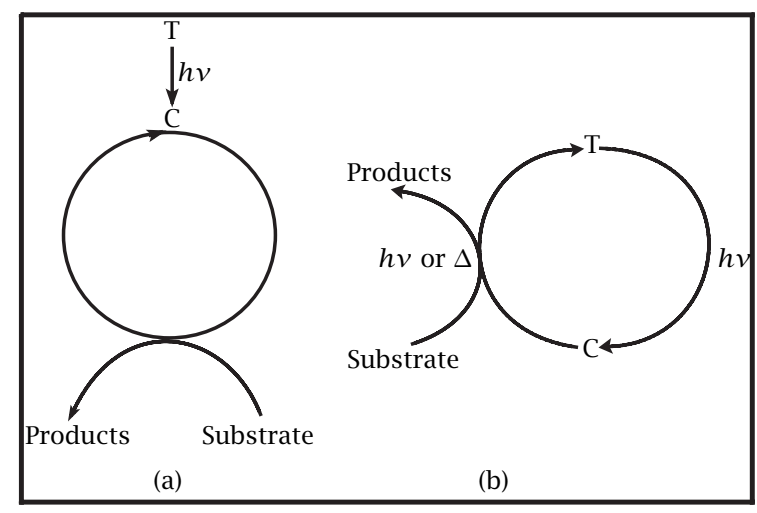

Figure 1. Proposed scheme of an example of (a) photogenerated catalysis which is catalytic in photons, and of (b) catalyzed photolysis which is non-catalytic in photons. Note that $T$ is the nominal catalyst and $C$ denotes the catalytic entity. (After refs. $[13,16]$ ).

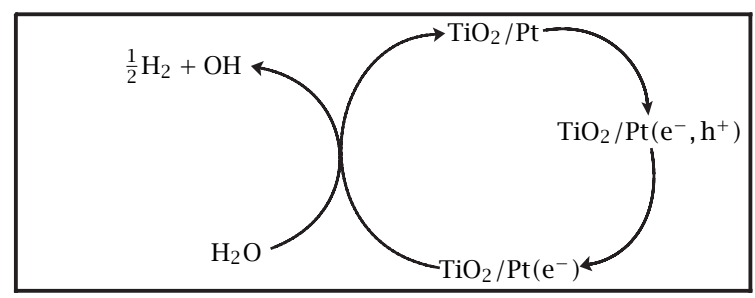

Scheme 1.

the dual role of light harvester and catalytic entity. An example is provided of the photocatalyzed reduction of water by the bifunctional catalyst $\mathrm{TiO}_{2} / \mathrm{Pt}$ in the presence of some electron donor D (Scheme 1). Several photoreactions that take place in the presence of semiconductor particles and that have been claimed to be photocatalytic may in fact be described more appropriately as semiconductor-assisted photoreactions [23].

\section{ISSUES WITH TURNOVER QUANTITIES}

The quantitative measure of photocatalytic activity of a solid photocatalyst is an essential quantity in heterogeneous photocatalytic solid/gas or solid/liquid chemistry, particularly in industrial processes where catalytic activities bear on process economics and thus on acceptance of a given process. Such measures are characteristically derived from process kinetics to express a rate referenced to the number of photocatalytic sites to infer how many times a catalytic cycle turnsover. Traditionally, this parameter is the turnover number (TON). Two other related parameters have sometimes been used and described [24-26]: (a) turnover rate (TOR) 
Table 1. Salomon's classification of photocatalysis and summary of various mechanism-specific labels [15].

\begin{tabular}{ll}
\hline Catalytic in Photons & Non-Catalytic in Photons \\
\hline Photogenerated catalysis & Catalyzed photolysis \\
\hline photoinduced catalytic reactions [18] & catalyzed photochemistry [17] \\
(Stoeichiometric photogenerated catalysis [15]) & catalyzed photoreactions [17, 20] \\
& sensitized photoreactions [17, 18] \\
& photosensitized reactions [21] \\
& photo-assisted catalysis [18, 22] \\
& (stoichiometric photogenerated catalysis [15]) \\
& substance-assisted photoreactions [23] \\
& substance-catalyzed photoreactions [23] \\
\hline
\end{tabular}

which designates the number of reagent molecules consumed or product molecules formed per surface active site per unit time, and (b) turnover frequency (TOF) which denotes the number of reactant molecules consumed, or product molecules formed, per active site per unit time (note the confusing similarity with turnover rate-however, see below). Laidler [24] noted that because turnover numbers (and by extension, turnover rates and turnover frequencies) vary with temperature, concentration and other experimental conditions, TON is not a useful quantity in kinetic work.

The expression sought to signify activity should serve two basic functions: (i) to establish whether a given process is catalytic or stoichiometric, and (ii) to provide a quantity to compare activities (unrelated to photons) of various catalysts for a given process under a set of conditions. A few decades ago an IUPAC article [27] pointed out that:

“... the turnover frequency, N, (commonly called the turnover number) defined, as in enzyme catalysis, as molecules reacting per active site in unit time can be a useful concept if employed with care. In view of the problems in measuring the number of active sites discussed in ..., it is important to specify exactly the means to express ... in terms of active sites. A realistic measure of such sites may be the number of surface metal atoms on a supported catalyst but in other cases estimation on the basis of a BET surface area may be the only readily available method. Of course, turnover numbers (like rates) must be reported at specified conditions of temperature, initial concentration (or initial pressure) and extent of reaction ..."

Not surprising, some workers (see e.g., [24-26, 28] have taken TON, TOR and TOF to refer to a singular description, namely that they describe the number of molecules reacted (or produced) per active site per unit time. In fact Boudart [28, 29] makes no distinction between turnover rate and turnover frequency.

Conceptually, however, we argue that the three quantities (1) turnover number, (2) turnover rate and (3) turnover frequency are distinct from one another. The problem (as we see it) originates from common usage in which turnover rate and turnover frequency have often been used interchangeably by catalycists, just as photochemists often make no distinction between quantum yield and quantum efficiency, and chemical kineticists often use the rate constant to mean the rate of a reaction.

Boudart [29] has deplored the usage of the quantity turnover number (TON) because it has become to be not a dimensionless number (as it should be) but a number expressed in units of time ${ }^{-1}$, i.e., a number that expresses a frequency. Thus, the equivalence often made by many workers between turnover number and turnover frequency. For some (see e.g., [30]), turnover number is understood to be a quantity that does not involve the element of time.

It must be stressed that only in a very specific case are the turnover rate and the turnover frequency identical. Just as a unique quantum yield is described for a zero-order process, equivalence of TOR and TON may occur solely for zero-order processes and for small conversion of reactants (initial rates).

For a semiconductor-based heterogeneous photocatalytic process, a description of the number of photocatalytic active sites and thus of the measure of activity of a photocatalyst is rendered difficult as photons can easily generate new active sites on the semiconductor particle, not to mention the possible changes in the surface adsorption/desorption characteristics. Added to this, there is a likely possibility that active and inactive sites switch identities during a photocatalytic sequence and that activity during a photocatalytic process may then have a different meaning for different steps of the process [31]. For example, in a photocatalyzed oxidation of an organic substrate involving the photocatalyst $\mathrm{TiO}_{2}$, oxidation may be mediated by a $\bullet \mathrm{OH}$ radical at some surface site on the particle. Once oxidation has occurred at that site, this particular surface site becomes extinct and is no longer active until such time as the site has been reconstructed and another $\bullet \mathrm{OH}$ radical formed on that very same site.

Despite the above issues, it is nonetheless useful to report a turnover quantity in heterogeneous 
solid/liquid or solid/gas photocatalysis (to paraphrase from Boudart [29]) so (a) that the quantity can be reproduced in various laboratories, (b) that it can disclose whether a given process is truly photocatalytic, and (c) that it can prove useful in assessing new materials as photocatalysts.

Though much has been written on catalysis (see for example references in [29]), it is nonetheless worthwhile to recall some of the major steps of the (photo)catalytic process in a heterogeneous phase.

Where the photocatalyst (or catalyst) is a solid material, the following events need to take place [32]: (i) the molecule is adsorbed on the particle surface; (ii) the molecule undergoes chemical transformation while visiting several reaction surface sites by surface diffusion; and (iii) the intermediate or product molecule is subsequently desorbed to the gas phase or to the condensed phase.

A closer scrutiny of the adsorption step reveals that if the reactant molecule or any subsequent intermediate product is strongly chemisorbed, i.e., has formed strong chemical bonds with the surface atoms at the site, no photocatalysis will be possible. The process is stoichiometric because one molecule of product has formed per surface active site $(\mathrm{TON}=1)$, signifying that the reaction has turned only once on that site. Moreover, if during the photocatalytic process the reactant molecule is strongly (chemically) bonded to surface atoms, the photocatalytic site becomes inactive and said to be poisoned. By contrast, if (chemical) bonding interactions are too weak, the substrate is poorly adsorbed and there will be no opportunity for chemical bond rupture and bond making, an integral part of any catalytic process [32]. Thus, interactions between the reactant molecule and the photocatalyst's surface site must be such that bond breaking and bond making can take place within the residence time of the intermediate(s), and that desorption/adsorption can occur.

In heterogeneous photocatalysis a primary difficulty with describing the turnover number or the turnover rate is how to specify the number of surface active sites. It has become common practice (albeit of dubious usefulness) to substitute this quantity by the BET total surface area $\left(\mathrm{m}^{2}\right.$ or $\left.\mathrm{cm}^{2}\right)$ or by the specific surface area $\left(\mathrm{m}^{2} \mathrm{~g}^{-1}\right.$ or $\left.\mathrm{cm}^{2} \mathrm{~g}^{-1}\right)$ of the catalyst particles, as determined by physical adsorption of nitrogen or argon at low temperatures. The use of the usual "BrunauerEmmett-Teller (BET)" surface area in lieu of the number of active sites is tenuous, since the latter is seldom known in catalysis and much less known (if at all) in heterogeneous photocatalysis. In some cases, however, the number of active sites may be determined by kinetic measurements of gas photoadsorption (stoichiometric process) when the conditions of uniform irradiation of the catalyst surface and maximum surface coverage are satisfied; such sites may be taken to reflect the concentration of surface centers. The number of such sites is known for electron and hole centers on $\mathrm{ZnO}, \mathrm{TiO}_{2}$, $\mathrm{BeO}$, $\mathrm{MgAl}_{2} \mathrm{O}_{4}$ and $\mathrm{SiO}_{2} / \mathrm{TiO}_{2}$ (and some others); they range between $10^{10}$ and $10^{12}$ centers $\mathrm{cm}^{-2}$ [33]. It must be emphasized, however, that the BET surface area reflects the number of adsorption sites and not necessarily the number of catalytically active sites. On this point, Somorjai [32] noted that only about $10 \%$ or less of the surface sites may be active in any given catalytic reaction/process, and that the specific turnover number is only a conservative estimate of the actual turnover. In other cases, the surface density of $\mathrm{OH}^{-}$groups $\left(10^{14}\right.$ to $10^{15} \mathrm{~cm}^{-2}$ for $\mathrm{TiO}_{2}$ ) has been used in lieu of the surface area to express catalytic activity [33, 34]. However, this usage also did not address the real issue. The turnover number that is estimated in this manner also represents a lower limit.

Contrary to the other two turnover quantities, turnover frequency (TOF) requires neither the knowledge of the surface area [32], nor the number of surface photocatalytically active sites. TOF increases with increasing active surface area and thus may be different from one batch of a catalyst to another and between various catalysts for the same reaction/process. Note that both TOR and TOF may be less than unity. When the turnover quantity depends on the surface characteristics (e.g., number of active sites) it represents only a conservative estimate. It must be emphasized that for various reasons an active site or photocatalyst will have a finite lifetime, one reason being inactivation through poisoning by impurities.

Determination of the turnover quantities TOR and TON in a heterogeneous system necessitates that the concentration of the surface centers (S) in the original state of the photocatalyst be taken into account, and not the concentration of one of its excited states (e.g., $\mathrm{S}^{+}$) [10]. This may simplify the experimental determination of turnover quantities. However, in most cases, assessment of the concentration of surface reactive centers remains a huge challenge, except in some particular cases (see ref. [33]). Another problem connected with turnover quantities is that all are likely to depend on light irradiance (or photon flow), an effect yet to be determined and verified experimentally. Indeed, although light irradiance incident on the reactor can easily be measured by actinometry, the extent of scattering and absorption keeps changing from particle to particle and are different at different points of the reactor, not least of which is their dependence on reactor geometry. This requires complex calculations of the light irradiance distribution in a given reactor. Also, it is worth noting that the irradiated surface area is not necessarily equal to the total surface area of the catalyst, s. Thus, the practical determination of turnover quantities remains a very complex problem.

Nonetheless, on the basis of the above discussion and the more detailed earlier study [10], we propose that the turnover rate be taken simply as the number 
of molecules reacted or produced per active site per unit time $\left(^{2}\right)$ (units: molecules site ${ }^{-1}$ time $^{-1}$ ); turnover frequency as the number of molecules reacted or produced per unit time (units: molecules time ${ }^{-1}$ ); and, in accord with others [32], turnover number as a quantity that describes how many times a reaction or process turnsover at some active site (or at some catalyst molecule in homogeneous catalysis) integrated over time (units: molecules site $\left.{ }^{-1}\right) .\left({ }^{3}\right)$

We now return to some considerations on the terminology of photocatalysis following an earlier more detailed study [10] in which an attempt was made to provide a mathematical description of photocatalysis in a heterogeneous system.

\section{DESCRIPTION OF PHOTOCATALYSIS}

For a chemical reaction described by eq. (3) there may exist a corresponding catalytic process described by eq. (4), namely

$$
\begin{gathered}
\mathrm{A} \rightleftarrows \mathrm{B}, \\
\mathrm{A}+\mathrm{Cat} \rightleftarrows \mathrm{B}+\mathrm{Cat}
\end{gathered}
$$

The simplest description of a catalytic process is that catalysis occurs when addition of a catalyst (Cat) changes the rate of establishing an equilibrium state in reaction (4), as compared to the equilibrium state of reaction (3). After a single act of the reaction (or completion of the reaction), the catalyst can be separated in the same original state and in the same amount as before the reaction. This description requires no prior knowledge of the mechanism of any particular catalytic process. Of course, the more complex and more precise description of catalysis would state that the catalyst is intimately involved in the chemical steps. Clearly, the reaction pathway in the catalytic process of reaction (4) would be different from that of reaction (3). After completion of the reaction cycle that yields the products, the catalyst is regenerated into its original state. Note that there is no need to know the mechanistic details of the process except for the existence of interactions (e.g., adsorption in

$\left({ }^{2}\right)$ Somorjai [32] referred to this as a "specific turnover rate". ${ }^{3}$ ) The units of these turnover quantities, especially the TON, might confuse the reader as we had earlier noted, as did Boudart [29], that TON should be dimensionless. This problem is identical with the dimensions of the quantum yield, which is also a dimensionless parameter but indicating for example the number of molecules that have been consumed or produced in a process per photon absorbed by the substrate. To the extent that TON refers to the number of molecules converted per active site, the TON parameter can also be considered as dimensionless by extension of the quantum yield argument. In this regard then, TOR will have units of molecules site ${ }^{-1}$ time $^{-1}$ and TOF will have units of molecules time ${ }^{-1}$. heterogeneous photocatalysis) between reagents and catalyst. This notion is valid for acid-base catalysis, redox catalysis and biocatalysis (as well as for others). The reaction rate increases if the total activation energy in the catalytic process (eq. (4)) is lower than the corresponding energy in reaction (3). In thermal (catalytic) reactions, changes in the electronic configuration of the system occur following the regrouping of nuclei/fragments without transitions to electronic excited states.

As emphasized earlier, a definition of photocatalysis must also be general just as is the case of catalysis. Also, any description must be independent of any particular mechanism of the process. (Compare this assertion with the many labels listed in Table 1).

Transformation of chemistry to photochemistry occurs when a chemical reaction is induced by absorption of photons by some reagent A (eq. (5)):

$$
\mathrm{A}+h v \longrightarrow \mathrm{B}
$$

The corresponding photocatalyzed process is:

$$
\mathrm{A}+h v+\mathrm{Cat} \longrightarrow \mathrm{B}+\mathrm{Cat}
$$

Unlike thermal reaction (3), photoreaction (5) occurs through an excited electronic state of the reagents followed by the regrouping of the various nuclei/fragments. Typically, the photochemical reaction (5) is irreversible. If light is taken as a quasi-reagent in reaction (5) (as often done in mechanistic studies), the back reaction must follow the pathway:

$$
\mathrm{B} \longrightarrow \mathrm{A}+h v
$$

where $h v$ denotes photons of identical energy as those used in the forward reaction (5). Clearly, such back reaction is highly unlikely, and the process $\mathrm{B} \rightarrow \mathrm{A}$ must of necessity proceed by a different pathway.

For discussion, it is relevant to consider two different approaches to photocatalysis. The first approach sees the sequence $(3) \rightarrow(4) \rightarrow(6)$, that is from chemistry (eq. (3)) to catalysis (eq. (4)) to photocatalysis (eq. (6)). The second approach is $(3) \rightarrow(5) \rightarrow(6)$ (chemistry $\rightarrow$ photochemistry $\rightarrow$ photocatalysis). From this point of view, the problem of defining photocatalysis is associated with one's approach(es) to photocatalysis. Indeed, using the approach $(3) \rightarrow(4) \rightarrow(6)$ we may consider photocatalysis as catalysis of a thermal reaction (eq. (3)) by an excited state of the catalyst produced as a result of light absorption by the catalyst. Thus, the role of light is to form the active (excited) state of the catalyst or to produce more active sites on its surface (for a heterogeneous system) during photoexcitation. An example of such a process is the photoinduced isotope exchange of oxygen and hydrogen on photogenerated surface hole centers $\left(\mathrm{O}^{-} \mathrm{s}\right)$ on metal-oxide semiconductors and dielectrics. Oxidation of organic 
compounds over $\mathrm{TiO}_{2}$ particles is yet another example if absorption of photons by $\mathrm{TiO}_{2}$ generates the active state of the photocatalyst, in this case titanium dioxide.

Subsequent to light absorption by the catalyst, surface photochemical processes may be treated as catalysis of a photoreaction if light is considered as one of the reagents. In this case, there is catalysis of the photochemical reaction (5) with changes of the reaction pathway, in which the first step is interaction of the catalyst with the reagent (light) to form an intermediate species (e.g., an excited state of the catalyst), which subsequently reacts with another reagent (molecules A) to form the final reaction product (B).

Clearly, a definition of photocatalysis must be quite general to cover all particular processes (considered above and no doubt many others) when there is an acceleration of reactions equivalent to reaction (4) with the participation of light, or of reactions analogous to reaction (5). At the end of the reaction cycle, the photocatalyst is regenerated into its original state. In (thermal) catalysis, such kinetic parameters as turnover rate, turnover frequency and turnover number are used to determine whether a given surface reaction is catalytic. Common processes to determine the corresponding kinetic parameters to demonstrate whether photocatalysis is catalytic have been treated by Emeline and coworkers [10]. Next we compare/contrast catalyzed photolysis and photogenerated catalysis.

4.1. Catalyzed photolysis. We examine a simple photochemical process, summarized by mechanism I, that takes place on an inactive surface of a photocatalyst when light is absorbed by an adsorbed substrate.

\section{Mechanism I.}

$$
\begin{aligned}
& \{1\} \mathrm{M}+\mathrm{S} \longrightarrow \mathrm{M}_{\mathrm{ads}} \\
& \{2\} \mathrm{M}_{\mathrm{ads}} \longrightarrow \mathrm{M}+\mathrm{S} \\
& \{3\} \mathrm{M}_{\mathrm{ads}}+h v \longrightarrow \mathrm{M}^{*} \text { ads } \\
& \{4\} \mathrm{M}^{*} \text { ads } \longrightarrow \mathrm{M}_{\mathrm{ads}} \\
& \{5\} \mathrm{M}^{*} \text { ads } \longrightarrow \mathrm{S}+\text { products }
\end{aligned}
$$

Stage $\{1\}$ describes the adsorption of reagent $M$ on the surface site $\mathrm{S}$ of the catalyst while stage $\{2\}$ reflects desorption of adsorbed molecules $\mathrm{M}_{\mathrm{ads}}$. Both processes lead to the establishment of an adsorption/desorption Langmuir equilibrium whose constant is $\mathrm{K}=k_{1} / k_{2}$. Stage $\{3\}$ is photoexcitation of adsorbed molecules to form some appropriate excited state $\mathrm{M}^{*}$ ads followed by the spontaneous decay of excitation (stage $\{4\}$ ) and chemical reaction (stage $\{5\}$ ) to regenerate the original state of the catalyst surface $S$, as required by the definition of catalysis (otherwise mechanism I would simply describe a surface stoichiometric photoreaction). All the kinetic parameters (TOR, TOF, TON) are determined under steady-state conditions in all mechanisms considered I-III, i.e., when the concentrations [M] and $[\mathrm{S}]$, and photon flow $\rho$ are constant.
Introducing the quantum yield of product formation $\Phi$, and the equilibrium coverage of the surface of the catalyst by adsorbed molecules $\theta$ such that $\theta=\left[\mathrm{M}^{\prime}{ }_{\text {ads }}\right] /\left[\mathrm{S}_{\mathrm{o}}\right]$ in the dark at concentration $[\mathrm{M}]$, the turnover quantities are given by [10]:

$$
\begin{aligned}
& \text { TOF } \propto \Phi k_{3} \rho\left[\mathrm{M}_{\mathrm{ads}}\right] \\
& \text { TOR } \propto \Phi k_{3} \rho \theta \\
& \text { TON } \propto \Phi \mathrm{t} k_{3} \rho \theta
\end{aligned}
$$

To the extent that TON yields information about "photocatalysis" being catalytic, the efficiency and activity of the photocatalytic process are better characterized by the quantum yield. The greater the quantum yield is, the greater are the three turnovers. Note that the same expression for the quantum yield is obtained for the photochemical reaction if in stages $\{3\}-\{5\}$ we substitute $\mathrm{M}_{\mathrm{ads}}$ with $\mathrm{M}$ and $\mathrm{M}^{*}$ ads with $\mathrm{M}^{*}$. The rate of such a photochemical process in homogeneous media (primed parameters) at the same concentration $[\mathrm{M}]$ is given by eq. (11):

$$
\frac{\mathrm{d} C}{\mathrm{~d} t}=\frac{k^{\prime}{ }_{3} k^{\prime}{ }_{5} \rho[\mathrm{M}]}{k^{\prime}{ }_{4}+k^{\prime}{ }_{5}}
$$

Thus, acceleration of the photoreaction in a heterogeneous system (over a homogeneous one) occurs when changes in the structure of the adsorbed molecule cause an increase in the photon absorption crosssection $k_{3}>k^{\prime}{ }_{3}$, in the stabilization of the excited state of the molecule $k_{4}<k^{\prime}{ }_{4}$, and a decrease of the activation energy of the reaction $k_{5}>k^{\prime}{ }_{5}$. Of course, the rate will also depend on the number of adsorption sites on the surface of the catalyst. The overall condition to observe acceleration is then given by:

$$
\frac{k_{3} \Phi}{k^{\prime}{ }_{3} \Phi^{\prime}} \times \frac{\left[\mathrm{M}_{\mathrm{ads}}\right]}{\mathrm{K}[\mathrm{M}]}>1
$$

Consequently, if the absorption spectra of adsorbed and free molecules are similar (i.e., if $k_{3} \sim k^{\prime}$ ), then in order to observe photocatalytic acceleration the quantum yield of the heterogeneous photoreaction must be greater than the corresponding quantum yield of the homogeneous process, that is $\Phi>\Phi^{\prime}$ (see eq. (12)).

4.2. Photogenerated catalysis-the LangmuirHinshelwood process. The case of a LangmuirHinshelwood type photocatalytic reaction, which occurs at a photochemically active surface when light is absorbed by the catalyst and leads to the generation of surface electrons $\left(\mathrm{e}^{-}\right)$and holes, $\left(\mathrm{h}^{+}\right)$, is described by mechanism II:

Mechanism II.

\{6\} $\mathrm{M}+\mathrm{S} \longrightarrow \mathrm{M}_{\mathrm{ads}}$

\{7\} $\mathrm{M}_{\mathrm{ads}} \longrightarrow \mathrm{M}+\mathrm{S}$

$\{8\} \quad$ Cat $+\mathrm{h} v \longrightarrow \mathrm{e}^{-}+\mathrm{h}^{+}$ 


$$
\begin{aligned}
& \{9\} \quad \mathrm{M}_{\mathrm{ads}}+\mathrm{h}^{+} \longrightarrow \mathrm{M}_{\mathrm{ads}}{ }^{+} \\
& \{10\} \quad \mathrm{M}_{\mathrm{ads}}{ }^{+}+\mathrm{e}^{-} \longrightarrow \mathrm{M}_{\mathrm{ads}} \\
& \{11\} \quad \mathrm{M}_{\mathrm{ads}}{ }^{+} \longrightarrow \text { product }+\mathrm{S}
\end{aligned}
$$

Stages $\{6\}$ and $\{7\}$ are identical to $\{1\}$ and $\{2\}$ above, and both processes lead to the establishment of an adsorption/desorption Langmuir equilibrium with constant $\mathrm{K}=k_{6} / k_{7}$. Stage $\{8\}$ reflects the photoexcitation of the catalyst producing electrons and holes. Stage $\{9\}$ describes carrier (hole) trapping by the adsorbed molecule to form a reactive radical state, whose decay occurs through recombination with an electron described by stage $\{10\}$. Stage $\{11\}$ is the chemical reaction that yields the products and regenerates the original state of the catalyst surface, S. In this kinetic approach, the surface concentrations of holes and electrons for a uniform generation of charge carriers are given by:

$$
\left[\mathrm{h}^{+}\right]=\alpha_{\mathrm{h}} \rho \tau_{\mathrm{h}}
$$

and

$$
\left[\mathrm{e}^{-}\right]=\alpha_{\mathrm{e}} \rho \tau_{\mathrm{e}}
$$

where $\alpha_{\mathrm{h}}$ and $\alpha_{\mathrm{e}}$ are the absorption coefficients of the hole and electron absorption bands, $\rho$ is the photon flow, and $\tau_{\mathrm{h}}$ and $\tau_{\mathrm{e}}$ are the lifetimes of holes and electrons, respectively. One disadvantage of the kinetic approach is that it considers a spatially uniform generation of carriers in the bulk of the catalyst (i.e., $\alpha \rho=$ const) and there is no diffusion limitation for carrier motion. A more detailed description uses a nonuniform generation of carriers. Such an expression for the concentration of surface carriers was reported earlier [35].

The process described by mechanism II can be treated as a photochemical reaction on the surface of a solid (the catalyst Cat) whose role is to absorb light. Subsequent charge carrier transfer to the adsorbed molecules produces an ionized state of the adsorbed molecules as might also occur by direct interaction between the adsorbed molecules and light (not shown in mechanism II). The excitation rate in mechanism II, $k_{9}\left[\mathrm{~h}^{+}\right]=k_{9} \alpha_{\mathrm{h}} \rho \tau_{\mathrm{h}}$, is analogous to the rate $k_{3} \rho$ in mechanism I. In mechanism II, the photocatalyst also takes part in the deactivation process such that $k_{10}\left[\mathrm{e}^{-}\right]$ (or $k_{10}$ for thermal ionization of the adsorbed molecule) corresponds to $k_{4}$ in mechanism I. Note that the "light inert" catalyst in mechanism I also plays a role in the decay step (stage $\{4\}$ ) by changing the probability of deactivation.

In the current context the turnover quantities are given by

$$
\begin{aligned}
\mathrm{TOF} & =\mathrm{V} \alpha \rho \Phi \\
\mathrm{TOR} & =\frac{\alpha \rho \Phi}{\left[\mathrm{S}_{\mathrm{O}}\right]} \times \frac{\mathrm{V}}{\mathrm{s}}
\end{aligned}
$$

$$
\mathrm{TON}=t \frac{\alpha \rho \Phi}{\left[\mathrm{S}_{\mathrm{o}}\right]} \times \frac{\mathrm{V}}{\mathrm{s}}
$$

where $\mathrm{V}$ is the volume of the catalyst and $\mathrm{s}$ is the total surface area of the catalyst. Hence, just as in mechanism I, all the turnover quantities are associated with the quantum yield of the photoprocess. Once again the activity and efficiency of the photocatalyst scale with $\Phi$.

When free surface electrons and holes are the reactive centers [36], TOR and TON are given by,

$$
\begin{aligned}
& \text { TOR } \propto \frac{k_{6} k_{9} k_{11}\left[\mathrm{M}_{\mathrm{ads}}\right]}{k_{10}\left[\mathrm{e}^{-}\right]+k_{11}}=\frac{\mathrm{V}}{\mathrm{s}} \tau_{\mathrm{h}} \Phi \\
& \mathrm{TON} \propto t \frac{\mathrm{V}}{\mathrm{s}} \tau_{\mathrm{h}} \Phi
\end{aligned}
$$

In this case, catalysis occurs from the excited state of the catalyst and neither TOR nor TON depend on light irradiance, unless the decay of the ionized state of the adsorbed molecule is caused by recombination or photoionization, and $k_{10}\left[\mathrm{e}^{-}\right] \sim k_{11}$.

4.3. Photogenerated catalysis-the Eley-Rideal process. Another possible heterogeneous photochemical process may be observed when the catalyst is photoexcited and no pre-adsorption of M occurs, as described by

\section{Mechanism III.}

$$
\begin{aligned}
& \begin{array}{l}
\{8\} \text { Cat }+h v \rightarrow \mathrm{e}^{-}+\mathrm{h}^{+} \\
\{12\} \mathrm{S}+\mathrm{h}^{+} \longrightarrow \mathrm{S}^{+}
\end{array} \\
& \begin{aligned}
\{13\} \mathrm{S}^{+}+\mathrm{e}^{-} \longrightarrow \mathrm{S} \\
\quad\left(\{13 \mathrm{a}\} \mathrm{S}^{+} \longrightarrow \mathrm{S}+\mathrm{h}^{+}\right)
\end{aligned} \\
& \quad\left(\{13 \mathrm{~b}\} \mathrm{S}^{+}+h v \longrightarrow \mathrm{S}+\mathrm{h}^{+}\right) \\
& \{14\} \mathrm{S}^{+}+\mathrm{M} \rightarrow(\mathrm{S}-\mathrm{M})^{+} \\
& \{15\} \quad(\mathrm{S}-\mathrm{M})^{+} \longrightarrow \mathrm{S}+\text { products } \\
& \quad\left(\text { or }\{15 \mathrm{a}\}(\mathrm{S}-\mathrm{M})^{+} \longrightarrow \mathrm{S}^{+}+\text {product }\right)
\end{aligned}
$$

As in mechanism II, stage $\{8\}$ corresponds to the photogeneration of free carriers. Stage $\{12\}$ describes the trapping of carriers (in this particular case, holes) by surface defects (i.e., "potential” surface active centers) $S$ to produce surface active centers $\mathrm{S}^{+}$; stage $\{13\}$ represents the "physical" decay pathway of surface active centers through recombination with charge carriers of the opposite sign, in this case electrons; it may also be a first-order thermal deactivation process (stage (13a)) or a second-order photoionization process (stage $\{13 \mathrm{~b}\})$. Stage $\{14\}$ is a chemical reaction (chemisorption) which yields the intermediate species $(\mathrm{S}-\mathrm{M})^{+}$followed by secondary reactions to produce the photoreaction products (stage $\{15\}$ ). Note the difference between stage $\{15\}$ and $\{15 \mathrm{a}\}$. In stage $\{15\}$ the original (ground) state $S$ of the photocatalyst is restored (quantum yield $\Phi<1$; the process is non-catalytic in photons), whereas in stage $\{15 \mathrm{a}\}$ the ionized state $\mathrm{S}^{+}$remains at the end of the reaction cycle (quantum yield $\Phi$ is greater than 1 ; 
the process is catalytic in photons). In the language of Salomon [15], the former is catalyzed photolysis, and the latter is photogenerated catalysis.

If the surface active centers in photogenerated catalysis are the ionized states $\mathrm{S}^{+}$then,

$$
\begin{aligned}
\text { TOF } & \propto \frac{\mathrm{d} C}{\mathrm{~d} t} \mathrm{~s} \\
\text { TOR } & \propto \frac{\mathrm{d} C / \mathrm{d} t}{\left[\mathrm{~S}^{+}\right]}=k_{14}[\mathrm{M}] \\
\text { TON } & \propto t k_{14}[\mathrm{M}]
\end{aligned}
$$

As in the case of surface photochemical reactions (mechanisms I and II), TON is greater than unity if turnover is determined for time $t<\tau$, where $\tau$ is the lifetime of the catalytic site. Hence the process can be said to be catalytic. It should be emphasized that TOR and TON are independent of light irradiance. They depend solely on reagent concentration, whereas the reaction rate and TOF behave differently. The latter two depend on light irradiance and (perhaps) on temperature for constant concentration of $\mathrm{M}$. Indeed, as $k_{13}\left[\mathrm{e}^{-}\right]\left(\right.$or $\left.k_{13}\right) \rightarrow \infty$, then $\mathrm{d} C / \mathrm{d} t \rightarrow 0$ and TOF $\rightarrow 0$. The physical sense of this behavior is that at high rate of "physical" decay, i.e., when $k_{13} \rightarrow \infty$, the concentration of the active centers $\left[\mathrm{S}^{+}\right] \rightarrow 0$, and so do the reaction rate and TOF. However, since TOR and TON are determined relative to a single center, as soon as this center becomes available the reaction cycle occurs and TOR and TON remain constant. Thus, TOR and TON reflect the activity of a given active center (ionized state) $\mathrm{S}^{+}$ but say nothing about the efficiency and activity of the catalyst. Moreover, for an effective physical decay (e.g., at sufficiently high light irradiance), the rates of both processes (photogenerated catalysis (stage (15a)) and catalyzed photolysis (stage (15))) become equal since they are determined only by the rate of photogeneration and (photo)decay of the ionized state; only one (or less) reaction cycle can occur during the lifetime of the active center.

When we consider catalysis of a photochemical reaction and assume that light is one of the reagents, all the excited states of the catalyst (electrons, holes, and $\mathrm{S}^{+}$ states) may be taken as intermediates of the catalytic photoreaction. The latter takes place by a different reaction pathway in contrast to the original photochemical reaction. The original state of the catalyst $S$ is restored at the end of the reaction, and the number of active centers is given by $S_{0}$. This is analogous to thermal catalysis. For example, the oxidation or photooxygenation reaction of molecule $\mathrm{M}$ might follow the path (reactions (23), (24)):

$$
\begin{gathered}
\mathrm{O}_{\mathrm{s}}+\mathrm{M} \longrightarrow \mathrm{MO} \uparrow+\mathrm{V}_{\mathrm{O}_{\mathrm{s}}} \\
\mathrm{V}_{\mathrm{O}_{\mathrm{s}}}+\frac{1}{2} \mathrm{O}_{2} \longrightarrow \mathrm{O}_{\mathrm{s}}
\end{gathered}
$$

The reactive center in such a process is neither the surface oxygen Os nor the oxygen vacancy $\mathrm{V}_{\mathrm{O}_{\mathrm{s}}}$, but is some cluster corresponding to oxygen vacancies either with or without oxygen in the cluster. By analogy, in photocatalysis there are surface centers (defects), which can be in a state with trapped carriers (i.e., an intermediate, just like surface oxygen in the previous example) or without trapped carriers. These centers are in fact the centers of photocatalysis.

To complete the consideration of the previous examples, we note that photogenerated catalysis (stage $\{15 \mathrm{a}\}$ ) does not require continuous irradiation since the excited state $\mathrm{S}^{+}$is reproduced at the end of each cycle. In this case, the excited state might be created during a pre-irradiation stage and there is no "physical" decay during the course of the reaction. As a result, stages $\{12\}$ and $\{13\}$ can be excluded from this consideration in mechanism III.

Even though the active (excited) state of the catalyst is restored in both catalyzed photolysis and photogenerated catalysis, the kinetic parameters are different [10], since we deal with a different excited state of the catalyst, i.e., effectively we deal with different catalysts. Indeed, under irradiation the state of the catalyst (e.g., a semiconductor) may be characterized by the splitting of the Fermi level into two quasi-Fermi levels (in catalyzed photolysis), whereas in photogenerated catalysis the state of the catalyst is characterized by a unique Fermi level (in the dark). This level may be shifted compared to the original state of the catalyst because of the possibility of having different excited states after pre-excitation of the catalyst.

\subsection{Further considerations of photocatalysis.} There is nothing dramatically special about photocatalysis. It is simply another type of catalysis joining, as it were, redox catalysis, acid-base catalysis, enzyme catalysis, thermal catalysis, and others. Consequently, we reemphasize that any description of photocatalysis must correspond to the general definition of catalysis. This said, it might be argued that the broad label photocatalysis might simply describe catalysis of a photochemical reaction. To discuss this proposition we consider the following.

If reaction (25) describes the catalyzed reaction between reagent $\mathrm{A}$ and reagent $\mathrm{B}$,

$$
\mathrm{A}+\mathrm{B}+\mathrm{Cat} \longleftrightarrow \text { products }+ \text { Cat }
$$

Then, in the absence of the catalyst (Cat) one is left with the chemical reaction (26):

$$
\mathrm{A}+\mathrm{B} \longrightarrow \text { products }
$$

which is no longer catalyzed by Cat as in reaction (25).

If this argument is carried further to the photocatalyzed reaction (27), removal of the catalyst

$$
\mathrm{A}+h v+\mathrm{Cat} \longrightarrow \text { products }+ \text { Cat }
$$


(Cat) leads to the photochemical reaction described by eq. (28).

$$
\mathrm{A}+h v \longrightarrow \text { products }
$$

By analogy, we deal with catalysis of a photochemical reaction by the catalyst (Cat) in its ground state. This notion is very important for determining the kinetic turnovers TOR and TON since their evaluation requires knowledge of which state of the catalyst (i.e., the state of the surface reactive centers) is being considered (see ref. [10]).

Some additional points are worth noting:

1. The photochemical reaction (28) takes place through the excited electronic state of the reagent, $A^{*}$, produced by the primary act of light absorption by reagent $A$, unlike chemical reaction (26), which occurs through the ground states of the reagents $A$ and $B$. In the photocatalyzed process (27), the reaction takes place after electronic photoexcitation of either the catalyst (Cat) or the adsorbed molecules $\left(\mathrm{A}_{\mathrm{ads}}\right)$, unlike catalysis, which involves only the ground electronic states (but thermally excited vibrational states).

and

2. The photocatalyzed reaction (eq. (27)) and the photochemical reaction (eq. (28)) describe irreversible processes, unlike thermal catalysis (eq. (25)) and the thermal chemical reaction (26).

In a broad sense then, the label photocatalysis describes a photochemical process in which the photocatalyst accelerates the process, as any catalyst must do according to the definition of catalysis.

Photoexcitation of the catalyst can be considered as changing the photochemical reaction pathway that is typical of a catalytic process. It can also lead to possible changes in the spectral range of photoexcitation relative to the non-catalytic photochemical reaction (eq. (28)) which, by analogy, can be considered as a change of the total activation energy because of the different reaction pathway in the catalytic process (eq. (25)). Indeed, photoexcitation of the catalyst to form its excited state (eq. (29)) in reaction (27) is analogous to the formation of the intermediate adsorption complex (eq. (30)) in thermal catalysis (eq. (25)), both of which are followed by secondary interaction steps with another reagent $\mathrm{A}$.

$$
\begin{aligned}
\mathrm{Cat}+h v & \longrightarrow \mathrm{Cat}^{*} \\
\mathrm{~B}+\mathrm{Cat} & \longrightarrow(\mathrm{Cat}-\mathrm{B})
\end{aligned}
$$

Thus, just as in thermal catalysis (eq. (25)), in which such an adsorption complex is not considered as the reactive center, the excited state of the photocatalyst in the photocatalytic process (27) is not a reactive center but an intermediate. Consequently, to determine TOR and TON in the proposed mechanisms I-III above one needs to consider as reaction centers the corresponding surface centers $S$ in the original state of the photocatalyst and not $\mathrm{S}^{+}$or $\mathrm{M}^{*}$ ads. As an example, we note the photocatalytic oxidation of organic compounds over $\mathrm{TiO}_{2}$ in aqueous media. In this case, the ground state of $\mathrm{S}$ centers corresponds to the surface $\mathrm{OH}^{-}$groups. It is these $\mathrm{OH}^{-}$groups that are the catalytic centers, whereas the $\bullet \mathrm{OH}$ radicals formed by hole trapping represent intermediate species. Note also that the original S state of surface-active centers is restored after the reaction is completed. In the example of $\mathrm{TiO}_{2}$, this restoration or reconstruction of the original state of the catalyst is achieved by dissociative adsorption of water on the particle surface. In this context, photocatalysis (i.e., catalyzed photolysis) is catalysis of a photochemical reaction by the original ground state of the catalyst prior to photoexcitation (see Table 1).

In photogenerated catalysis (see Figure 1(a)), preirradiation of the original state of the catalyst $(\mathrm{T})$ is the only means for the physical development of a new catalyst $(\mathrm{C})$ to create new centers; the corresponding catalytic process completely follows reaction (25). Similar changes to the state of the catalyst can be achieved by chemical doping, irradiation with ions or electrons, and additive coloration processes, among others. Thus photogenerated catalysis is thermal catalysis by a catalyst species produced by pre-irradiation (Figure 1(a)).

The principal difference between photogenerated catalysis and catalyzed photolysis can best be described by the following considerations.

Any photochemical reaction (eq. (28)) starts from light absorption by a reagent A to form an excited state of the molecule, $\mathrm{A}^{*}$ :

$$
\mathrm{A}+h v \longrightarrow \mathrm{A}^{*}
$$

There always exists a physical pathway (radiative and/or nonradiative) for relaxation of this state back to the ground state:

$$
\mathrm{A}^{*} \longrightarrow \mathrm{A}
$$

Accordingly, there is always a competition between chemical and physical pathways for decay of excited states. If the chemical pathway is inefficient, then the physical path will lead to relaxation of $\mathrm{A}^{*}$ to its ground electronic state A.

The same holds for catalyzed photolysis. Indeed, since catalyzed photolysis refers to catalysis of a photochemical reaction, there is a physical pathway for decay of the system back to its ground state. If the photocatalytic process occurred through photoexcitation of the catalyst, physical decay may occur through recombination, and/or through thermal and photo-ionization of the excited state of the surface centers, which 
ultimately lead to regeneration of the original state of the catalyst. There is no such process in the case of photogenerated catalysis. In the latter case, the state of the catalyst does not have a physical decay pathway and is the same before and after the reaction as in any thermal catalytic reaction. Note also that the catalytic process in photogenerated catalysis is reversible unlike in catalyzed photolysis.

There have also been attempts to contrast "photocatalysis" with "photosynthesis" on the basis of a thermodynamic analysis of the chemical transformations occurring in a process. An appropriate and universally acceptable definition of the notion "photosynthesis" has not heretofore appeared [37], except as it applied to "natural photosynthesis" (see Scheme 2). It reflects the production of complex organic substances starting from simpler compounds (e.g., carbon dioxide and water) by superior plants, algae and photosynthesizing bacteria at the cost of light energy absorbed by the chlorophyll and other photosynthesizing pigments (see below).

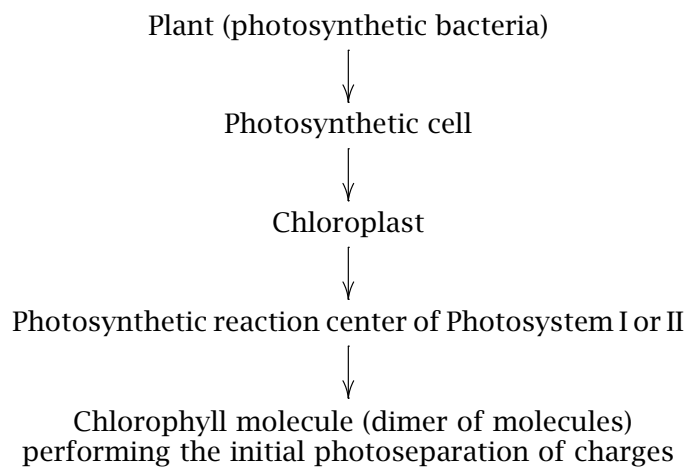

Scheme 2.

Others have used the term "photosynthesis" (as in artificial photosynthesis) to describe that process for which the thermodynamic potential of the reaction product(s) is greater than that of the reactants, whereas "photocatalysis" was attributed to the process in which the potential of the reaction product(s) is lower than that of the reactants [38].

To the extent that quantitative parameters are also considered in the proposed glossary, it would also be instructive to give some considerations [9] to the very important parameter, the quantum yield $\Phi$ of some given process.

\section{QUANTUM YIELDS}

Knowledge of the quantum yield (defined as the number of defined events which occur per photon absorbed by the system OR as the amount (mol) of reactant consumed or product formed per amount of photons (mol or einstein) absorbed [11]) has been central to homogeneous photochemistry. Photochemists have routinely determined quantum yields of reactant disappearance, product formation, light emission, and of various other events occurring in some photochemical process.

In heterogeneous photocatalysis, quantum yield has heretofore been taken to describe the number of molecules converted relative to the total number of photons incident on the reactor walls for some undefined reactor geometry and for polychromatic radiation. In fact, as in homogeneous photochemistry the quantum yield, $\Phi_{\lambda}$, must express the moles of reactant consumed or product formed in the bulk phase, $n$, to the amount (i.e., mol or einstein) of photons at wavelength $\lambda$ absorbed by the photocatalyst, $n_{\mathrm{ph}}$, (eqs. (33) and (34)).

$$
\Phi_{\lambda}=\frac{n}{n_{\mathrm{ph}}}
$$

Methods to determine $n_{\mathrm{ph}}$ have appeared [34, 39-41] Alternatively, the quantum yield could also be defined using the initial rate $R^{\text {in }}$ of the reaction and the rate of photons impinging on, and absorbed by the reaction system as is common practice in homogeneous photochemistry. Thus:

$$
\Phi_{\lambda}=\frac{R^{\text {in }}}{\rho_{\lambda}}
$$

where $\rho_{\lambda}$ is the photon flow at wavelength $\lambda$. Analogous descriptions have been proposed for heterogeneous systems [42-44]. In such systems, the relationships in eqs. (33) and (34) have been extended, modified and applied in an analogous fashion [18, 23, 30, 34, 39, 40] owing to reflection, scattering (see below), transmission (for transparent colloidal sols) and absorption by the suspended particulates, usage of the term quantum yield referenced to incident photons in heterogeneous photocatalysis has only engendered confusion. To increase the confusion, some workers have used such terms as apparent and true quantum yields. $\left({ }^{4}\right)$

To the extent that the numerator in eq. (34) expresses the rate of reaction, $\Phi_{\lambda}$ depends on the reactant concentration. However, as correctly noted by Braun and co-workers [42], and emphasized by Cabrera et al. [41], only for a zero-order reaction is $\Phi_{\lambda}$ uniquely defined at the given wavelength $\lambda$. In homogeneous photochemistry, the problem is normally overcome by determining $\Phi_{\lambda}$ at small (less than $\sim 10 \%$ ) conversions of reactants, a point not often respected in heterogeneous photocatalysis where the focus is usually complete mineralization (100\% transformation) of the substrate, at least in studies of environmental interest that focus on the total elimination of organic pollutants in water.

$\left({ }^{4}\right)$ Usage of the prefixes apparent and true when describing quantum yields (and other parameters) is discouraged as they simply engender much confusion and add nothing to the definitions. 
Additional considerations suggest that the photochemically defined quantum yield would be difficult to describe experimentally in heterogeneous media [41] particularly for complex reactor geometries. Consequently, so-called quantum yields thus far reported in the literature have been the so-called "apparent" or lower limits of the actual quantum yield, since scattered light has not been accounted for [44]. In defining eqs. (33) and (34), it must also be recognized that photooxidations take place on the surface of the solid catalyst and thus the catalytic properties of the catalyst surface are important as the course of reactions depends highly on the characteristics of the surface on light activation. This calls attention to the necessity of reporting the characteristics of the photocatalyst [23, 39, 44].

The numerator in eq. (34) expresses the rate of a catalyzed heterogeneous reaction in heterogeneous photocatalysis, which is related to the number of catalytically surface active sites [23]; unfortunately, these are not experimentally attainable [45]. To bypass this difficulty, the number of active sites has often been replaced [28] by (i) the surface area of the catalyst, (ii) the mass of the catalyst or (iii) by the number of surface $\mathrm{OH}^{-}$groups on a photocatalyst such as $\mathrm{TiO}_{2}[34,39,40]$. None of these suggestions, however, can describe the actual heterogeneous rate since measuring the surface area for a somewhat porous catalyst (for example) comprises both the external and internal surfaces [33]; the internal surface may not be useful in some catalytic events. Also, not all the surface sites occupied by $\mathrm{OH}^{-}$groups are necessarily catalytically active [45]. Finally, depending on the reactor geometry, particle aggregation, and stirring, not all the BET catalyst surface (Brunauer-EmmettTeller measurements in the dry state) may be accessible to the substrate being photoconverted.

Therefore a simple alternative method of comparing process efficiencies for equal absorption of photons was proposed $[8,9]$ for heterogeneous photocatalysis: relative photonic efficiency.

The term photonic efficiency ( $\xi$ ) had been suggested [46] to describe the number (or mols) of reactant molecules transformed or product molecules $n$ formed divided by the number or einsteins of photons at a given wavelength incident on the reactor cell (flat parallel windows) $n_{\mathrm{ph}}$. Alternatively, the photonic efficiency can also be described by relating the initial rate of the event to the rate of incident photons reaching the reactor as obtained by actinometry. The use of the term photonic efficiency, in lieu of "quantum yields", avoided confusion between heterogeneous and homogeneous photochemistry $[46,47]$. Nonetheless, this parameter has proven useful in describing the method to determine quantum yields through the limiting photonic efficiency $\xi_{\text {lim }}$ approach $[8,9]$.

This limiting photonic efficiency (see eq. (35)) provides a means to estimate quantum yields in an otherwise very complex medium. Taking up the photonic efficiency in the form:

$$
\xi=\frac{\xi_{\lim } \mathrm{Q}[\text { Cat }]}{\left(\xi_{\lim }+\mathrm{Q}[\text { Cat }]\right)}=\frac{R^{\text {in }}}{\rho_{\lambda}}
$$

for the case where $\mathrm{Q}[\mathrm{Cat}] \gg \xi_{\text {lim }}$, i.e., for high loading in photocatalyst Cat, one obtains $\xi=\xi_{\text {lim }}=R^{\text {in }} / \rho_{\lambda}$, which is exactly the definition of the quantum yield $\Phi$ (see eq. (34)) if all the photons impinging on the reactor system $\rho_{\lambda}$ are absorbed by the photocatalyst Cat at high loadings. Under these conditions, the limiting photonic efficiency for irradiation at a given wavelength $\lambda$ is the quantum yield of the process at the same wavelength; that is $\xi_{\lim }=\Phi_{\lambda}$. Note that if the $R^{\text {in }}$ data were obtained under broadband radiation in the wavelength range $\lambda_{1}$ to $\lambda_{2}$ and if the integrated photon flow were determined in the same wavelength range, then $\xi_{\text {lim }}=\Phi_{\text {poly }}$.

To avoid unnecessary errors and the necessity of stipulating reactor geometry and light source, together with the properties (e.g., size, surface area) of the photocatalyst material used, an earlier suggested protocol [45-47] further defined an efficiency that could be used to compare experiments within the same laboratory or with other laboratories and that would be reactor-independent: the relative photonic efficiency, symbolized as $\xi_{\mathrm{r}}$ and related to an acceptable standard process, a standard photocatalyst material [47], and a standard "secondary actinometer" in photocatalyzed processes. In the experimental description of a relative photonic efficiency [45-47], reactor geometry, light source, and photocatalyst properties remained constant in assessing $\xi_{\mathrm{r}}$ [46].

The concept of relative photonic efficiency avoided unnecessary confusion with the terminology appropriately defined in homogeneous photochemistry when an appropriate quantum yield is experimentally unattainable (however, see refs. [8, 9]). Moreover, $\xi_{\text {r could be ap- }}$ plied to whatever heterogeneous medium used: (i) for dispersions, (ii) for cases where the photocatalyst is immobilized on a support, and (iii) to solid/gas and solid/solution media.

In a practical application involving the photodegradation of phenolic substances, these relative process efficiencies were obtained by relating the initial rate of substrate degradation, $R^{\text {in }}$ (substrate), to the initial rate of phenol degradation, $R^{\text {in }}$ (phenol), for constant incident photon flow $\rho_{\lambda}$ reaching the reactor (note that the same reactor and reactor geometry must be used for both the substrate and phenol). Hence,

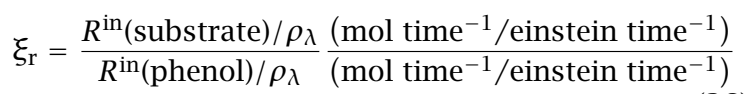

or

$$
\xi_{\mathrm{r}}=\frac{R^{\mathrm{in}}(\text { substrate })}{R^{\text {in }}(\text { phenol })} \quad \text { (dimensionless) }
$$

where both (initial) rates were obtained under 
otherwise identical conditions. To be useful, $\xi_{\mathrm{r}}$ values must not depend on light irradiance and reactor geometry, and on such other parameters as $\mathrm{pH}$, photocatalyst loading, substrate concentration, and temperature.

The glossary of terms proposed below is based on the above considerations (see also [4]) and is totally consistent with the earlier "Glossary of Terms used in Photochemistry" [5, 6]. Terms are also included that describe the phenomenon of radiation catalysis, a process generated by absorption of ionizing quanta (such as rigid UV-, X-ray, and $\gamma$-quanta or rapid $\alpha, \beta$-particles, etc.) and which results in a primary nonselective excitation of a catalyst (see, e.g., [48]).

\section{GLOSSARY OF TERMS AND DEFINITIONS}

\section{Absorbance $(A)$}

The logarithm to the base 10 of the ratio of the spectral radiant power of incident, essentially monochromatic radiation $\left(I=\int_{\lambda} I_{\lambda} \mathrm{d} \lambda\right)$ to the radiant power $\left(P_{\lambda}\right)$ of transmitted radiation:

$$
A=\log \left(\frac{P_{\lambda}^{o}}{P_{\lambda}}\right)=-\log T
$$

In practice, absorbance is the logarithm to the base 10 of the ratio of the spectral radiant power of light transmitted through the reference sample to that of the light transmitted through the solution, both observed in spectrally identical cells. $T$ is the (internal) transmittance. This definition supposes that all the incident light is either transmitted or absorbed, with reflection and/or scattering being negligible.

Traditionally, (spectral) radiant intensity, $I$, was used instead of spectral radiant power, $P_{\lambda}$, which is now the accepted form. (The terms absorbancy, extinction, and optical density should no longer be used).

\section{Absorptance (A)}

The fraction of light absorbed by a system equal to ( $1-T-R$ ), where $T$ is the transmittance and $R$ is the reflectance.

See absorbance.

\section{ABSORPTION (of electromagnetic radiation)}

The transfer of energy from an electromagnetic field to an entity (molecular or otherwise).

AbSORPTION COEFFICIENT (decadic: a; Napierian: $\alpha$ )

In a homogeneous solution, it is the absorbance A divided by the optical pathlength, $l$.

$$
a=\frac{A}{l}=\left(\frac{1}{l}\right) \log \left(\frac{P_{\lambda}^{o}}{P_{\lambda}}\right)
$$

When using natural logarithms, the expression becomes

$$
\alpha=a \ln 10=\left(\frac{1}{l}\right) \ln \left(\frac{P_{\lambda}^{o}}{P_{\lambda}}\right)
$$

Since absorbance is a dimensionless quantity, the acceptable SI unit for $a$, and $\alpha$ is $\mathrm{m}^{-1}$, although $\mathrm{cm}^{-1}$ is also used.

The absorption coefficient is also a quantity defined by the Lambert-Bougher law for an optically solid plate.

$$
P_{(x)}=(1-R) P_{o} \exp (-\alpha x)
$$

where $P_{(x)}$ is the attenuated radiant (energy) flux (same as radiant power, $P$ ) at a distance $x$ of a given point in the bulk of the solid plate from its irradiated surface, $P_{O}$ is the incident radiant power, and $R$ is the reflectance. In the particular case of intrinsic absorption of light by a semiconductor or dielectric Beer's law is applied for isolated defects (centers) so that $\alpha$ can be represented as $\alpha=\sigma n$, where $\sigma$ is the absorption cross section $\left[\mathrm{cm}^{2}\right]$ and $n$ is the concentration of the centers [ $\left.\mathrm{cm}^{-3}\right]$

A method for determining the absorption coefficients of solid phase heterogeneous photocatalysts has been reported in ref. [20].

Note that this definition does not only apply to a solid plate, but it also applies to solids of any shape. This notwithstanding, the meaning of the absorption coefficient for solids is generally identical to that for a solution or a gaseous system. In the case of solids in a heterogeneous system, the only important issue is how the concentration is determined. In this regard, the concentration of absorption centers must be determined with respect to the volume of the solid particulate and not of the whole system.

Note also that the term extinction coefficient is no longer recommended.

\section{Absorption Cross-Section $(\sigma)$}

Operationally, it can be calculated as the absorption coefficient divided by the number of molecular (or otherwise) entities contained in a unit volume of the absorbing medium along the light path:

$$
\sigma=\frac{\alpha}{N}=\left[\frac{1}{(N l)}\right] \ln \left(\frac{P_{\lambda}^{\mathrm{o}}}{P_{\lambda}}\right)
$$

where $N$ is the number of molecular (or otherwise) entities per unit volume, $l$ is the optical pathlength and $\alpha$ is the Napierian absorption coefficient.

The relation between the absorption cross-section and the molar (decadic) absorption coefficient, $\varepsilon$ (units of $\left.\mathrm{M}^{-1} \mathrm{~cm}^{-1}\right)$ is

$$
\sigma=0.2303\left(\frac{\varepsilon}{N_{\mathrm{A}}}\right)=3.825 \times 10^{-24} \varepsilon
$$

where $\sigma$ is in $\mathrm{m}^{2}$ (or $\mathrm{cm}^{2}$ ) and $N_{\mathrm{A}}$ is Avogadro's number.

See attenuance, Beer-Lambert law.

\section{ACCUMUlation LAYER}

The situation that occurs when the (conduction and valence) bands of a semiconductor bend downward because the majority carriers accumulate near the surface. 
As well, it can refer to the sub-surface space wherein the surface charge, whose sign is opposite to the sign of the majority charge carriers, in the semiconductor leads to accumulation of the majority charge carriers.

See depletion layer, band bending, inversion layer.

\section{ACTINOMETER}

A chemical system or physical device, which determines the number of photons in a beam integrally or per unit time. This term is commonly applied to devices used in the UV and visible wavelength ranges. For example, solutions of ferrioxalate can be used as a chemical actinometer, whereas bolometers, thermopiles, and photodiodes are physical devices giving a reading that can be correlated to the number of photons detected.

\section{ACTINOMETRY}

The process by which the number of photons emitted from a radiation source is determined using an actinometer.

\section{ACTION SPECTRUM}

A plot of a relative biological or chemical photoresponse $(=\triangle y)$ per number of incident photons, against wavelength or energy of radiation under the same radiant power of light. This form of presentation is frequently used in studies of biological or solid state systems, where the nature of the absorbing species is unknown. The action spectrum is sometimes called spectral responsivity or sensitivity spectrum. The precise action spectrum is a plot of the spectral (photon or quantum) effectiveness. By contrast, a plot of the biological or chemical change or response per absorbed photon (quantum efficiency) versus wavelength is the efficiency spectrum.

To make the action spectrum correspond to the spectrum of photocatalyst activity, the experimental conditions for the linear dependence of the reaction rate on light intensity over the whole spectral range must be established, so as to prevent the dependence of the spectral curve on the changes of the efficiency of a chemical process with changes in light intensity.

See excitation spectrum.

\section{ACTIVE STATE OF A SURFACE PHOTOADSORPTION CENTER}

An electronically excited surface center, or a surface center (surface site) with a trapped photogenerated charge carrier, that interacts with atoms, molecules or ions at the solid/liquid, liquid/liquid, solid/gas, or solid/liquid interfaces with formation of chemisorbed species.

See also photoadsorption center, chemisorption.

\section{Active State Of A Surface Photocatalytic CENTER}

An electronically excited surface center, or a surface center (surface site) with a trapped photogenerated charge carrier, that interacts with atoms, molecules or ions at an interface with the formation of reaction products or intermediates.

See also photocatalytic center.

ADSORBATE (adsorbed species)

A molecule (ion or atom) adsorbed on the surface of a solid as a result of physical (physisorption) or chemical (chemisorption) interactions between the solid and the molecule.

\section{ADSORPTION}

The act by which an entity (molecule, atom or ion) adsorbs onto a surface through either a physical or chemical interaction between the entity and the surface.

See chemisorption, physisorption.

\section{AM(0) Sunlight}

The solar irradiance in space just above the Earth's atmosphere (air mass zero, AM0). Also called extraterrestrial global irradiance.

\section{AM(1) SUNLIGHT}

The solar irradiance traversing the atmosphere when the sun is in a position perpendicular to the Earth's surface. Also called terrestrial global irradiance.

\section{APPARENT LIFETIME}

See lifetime.

(Use of such a prefix as "apparent" is not recommended as it is devoid of physical meaning.)

\section{APPARENT QUANTUM YIELD}

The term photonic efficiency is recommended in lieu of apparent quantum yield when reference is made to the ratio of the number of photoinduced events to the number of photons incident on the reaction system.

See photonic efficiency.

\section{Artificial Photosynthesis}

An endoergic process by which light quanta and a photocatalyst (or photocatalysts) are used to prepare compounds and for which the Gibb's free energy is positive (i.e., $\Delta G>0$ ), contrary to a photocatalytic process for which $\Delta G<0$. As an example of such a process, consider the water splitting reaction to produce hydrogen and oxygen.

See also natural photosynthesis, and photoelectrosynthesis.

\section{ATtenuance $(D)$}

The logarithm to the base 10 of the ratio of the transmittance $(T)$ :

$$
D=-\log T=\log \left(\frac{P_{\lambda}^{o}}{P_{\lambda}}\right)
$$

Attenuance reduces to absorbance when the incident beam is either transmitted or absorbed only, but neither reflected nor scattered.

\section{AtTenuance Filter}

An optical device (filter) that reduces the radiant power of a light beam by a constant factor over all 
wavelengths within its operating range. It is sometimes called a neutral density filter or attenuator.

\section{BAND BENDING}

Bending of the (valence or conduction) bands in semiconductors at the surface owing to the existence of the surface charge potential formed as a result of adsorption of (donor or acceptor) molecules, or of a different distribution of defects in the sub-surface region compared to the distribution in the bulk.

\section{BANDGAP}

The energy gap between the bottom of the conduction band and the top of the valence band in semiconductors or dielectrics.

\section{BANDGAP ENERGY $\left(E_{\mathrm{bg}}\right)$}

The energy difference between the bottom of the conduction band and the top of the valence band in semiconductors and insulators.

See intrinsic absorption, extrinsic absorption.

\section{BANDPASS FILTER}

An optical device, which permits the transmission of radiation within a specified wavelength range and does not permit transmission of radiation at higher or lower wavelengths. It can also be an interference filter.

See also cut-off filter.

\section{BAND-TO-BAND RECOMBINATION}

The recombination of charge carriers that occurs via a one-step electron transition from the conduction band to the valence band in a semiconductor or insulator.

\section{BATHOCRHOMIC SHIFT (Effect)}

Shift of a spectral band to lower frequencies (longer wavelengths) owing to the influence of substitution or a change in environment (e.g., solvent). It is informally referred to as a red shift and is opposite to hypsochromic shift.

BEER-LAMBERT LAW (or Beer-Lambert-Bouguer Law)

The absorbance of a collimated beam of monochromatic radiation in a homogeneous isotropic medium is proportional to the absorption pathlength, $l$, and to the concentration, $c$, or -in the gas phase- to the pressure of the absorbing species. This law holds only under the limitations of the Lambert Law and for absorbing species exhibiting no concentration or pressure dependent aggregation. The law can be expressed as:

$$
A=\log \left(\frac{P_{\lambda}^{o}}{P_{\lambda}}\right)=\varepsilon c l
$$

or

$$
P_{\lambda}=P_{\lambda}^{o} 10^{-\varepsilon c l}
$$

where the proportionality constant, $\varepsilon$, is the molar (decadic) absorption coefficient. For $l$ in $\mathrm{cm}$ and $c$ in mol $\mathrm{dm}^{-3}$ or $\mathrm{M}$, $\varepsilon$ will result in $\mathrm{dm}^{-3} \mathrm{~mol}^{-1} \mathrm{~cm}^{-1}$ or $\mathrm{M}^{-1}$ $\mathrm{cm}^{-1}$, which is a commonly used unit. The SI unit of $\varepsilon$ is $\mathrm{m}^{2} \mathrm{~mol}^{-1}$.

Note that spectral radiant power must be used because the Beer-Lambert law holds only if the spectral bandwidth of the light is narrow compared to the spectral linewidths in the spectrum.

See absorbance, attenuance, Lambert Law.

\section{BLACK-BODY-Like PHOTOCATAlytic REACTOR}

A reactor (spherical or cylindrical in shape) used to determine spectral quantum yields of photoadsorption and photocatalyzed reactions when the catalyst is in powdered form (as a dispersion). The reactor consists of a cell for powdered photocatalyst samples with a cavity and narrow inlet window to allow illuminating the surface of the solid particles inside the cell cavity. The incident photons are (nearly) all absorbed provided the thickness of the powder layer is sufficiently large.

Alternatively, for dispersions involving powdered catalysts contained in other reactors, a protocol has been given to determine quantum yields of photocatalyzed reactions (see refs. [8, 9]).

\section{BLEACHING}

In photochemistry this term refers to the loss of absorption or emission intensity. In semiconductor photochemistry and photophysics, it refers to the loss of color (loss of absorption) by the color centers.

\section{BLUE SHIFT}

Informal expression for hypsochromic shift.

\section{BRUNAUER-EMMETT-TELLER (BET) SURFACE AREA}

Specific surface area $\left(\mathrm{m}^{2} \mathrm{~g}^{-1}\right)$ determined from the adsorption isotherm obeying the mechanism of multilayer adsorption proposed by Brunauer, Emmett, and Teller. Usage of the value obtained from the adsorption isotherm of dinitrogen is recommended.

\section{CATAlysis}

A process in which a substance (the catalyst, Cat), through intimate interaction(s) with the reactant(s) and through a lower energy pathway, accelerates an otherwise thermodynamically favored but kinetically slow reaction with the catalyst fully regenerated quantitatively at the conclusion of the catalytic cycle. Thus, for a chemical reaction described by the reaction (47)c,

$$
\mathrm{A} \rightleftharpoons \mathrm{B}
$$

there may exist a corresponding catalytic process described by the reaction (48)d, namely

$$
\mathrm{A}+\mathrm{Cat} \rightleftarrows \mathrm{B}+\mathrm{Cat}
$$

Accordingly, the simplest description of a catalytic process is that catalysis occurs when addition of a catalyst (Cat) changes the rate of establishing the equilibrium state in reaction (48)d, as compared to the equilibrium state of reaction (47)c. After a single act of the 
reaction (or completion of the reaction), the catalyst can be separated in the same original state and in the same amount as before the reaction.

\section{CATALYSIS OF A PHOTOCHEMICAL REACTION}

See catalyzed photolysis and photocatalysis.

\section{CAtalyst}

See catalysis.

\section{CATAlyst PHOtOACTIVATION (photomodification)}

The generation or alteration of the catalytic activity (selectivity) of a substance after its interaction with light quanta.

\section{CATALYTIC SITES (in photocatalysis)}

The sites at which chemical transformations take place.

Note that in heterogeneous photocatalysis, the density or concentration of active sites is often unknown. This makes it difficult to express the reactions in terms of such sites. If the assumption can be made that the active sites are distributed over the entire (photo)catalyst surface, then the heterogeneous reactions can be described in terms of "per unit area". By lack of a better alternative, the generally accepted BET $\left(\mathrm{N}_{2}\right)$ surface area [49] could be used. It must be emphasized, however, that there is no correlation between the number of active sites and the BET $\left(\mathrm{N}_{2}\right)$ area. Nonetheless, the use of the latter may help to avoid confusion when no other alternatives exist. Quantities expressed in terms of "per unit $\left[\operatorname{BET}\left(\mathrm{N}_{2}\right)\right]$ area" should be denoted as areal. The notation areal also implies that we deal with a lower limit of the quantity.

\section{CATAlYZED PHOTOCHEMistry}

See catalyzed photolysis.

\section{CATALYZED PHOTORERACTIONS}

See catalyzed photolysis and photocatalysis.

\section{CATALYZED PHOTOLYSIS}

Catalyzed photolysis refers to catalysis of a photochemical reaction, for which there exists a physical pathway for the decay of the system back to its ground state. When the photocatalytic process occurs through photoexcitation of the catalyst, the physical decay may occur through recombination, and/or through thermal and photo-ionization of the excited state of the surface centers, which ultimately lead to regeneration of the original state of the catalyst. Note that catalyzed photolysis is not catalytic in photons, contrary to photogenerated catalysis (see Table 1 and Figure 1).

\section{CHARge CARRIER}

Electrons or holes usually produced on irradiating a semiconductor or insulator with appropriate energy through intrinsic absorption or extrinsic absorption of the actinic light.

\section{Chemical PATHWAy}

A photoexcited system that is converted to its (electronically) excited state represents the pathway of the system to relax to a lower energy state owing to a chemical reaction and formation of lower energy products.

\section{CHEMIEXCITATION}

Generation of electronically excited (molecular or otherwise) entities by a chemical reaction between reactants in their ground electronic states.

\section{Chemiluminescence}

Luminescence arising from electronically excited states or species produced by chemiexcitation.

\section{CHEMISORPTION}

Adsorption of an entity (molecule, atom or ion) onto a surface by way of a chemical interaction-usually dissociative adsorption-between a surface and the entity. An example is the dissociative adsorption of water onto a metal-oxide particle surface.

\section{CHROMOPHORE (group)}

That part of a molecular entity consisting of an atom or group of atoms in which the electronic transition responsible for a given spectral band takes place between two states localized in large part on this atom or group.

\section{COLOR CENTER}

As in solid-state physics, it is the long-lived deep trap with low efficiency of trapping a carrier of the opposite sign. Color centers are accumulated in irradiated solids and give rise to photoinduced extrinsic absorption bands (photoinduced color) in semiconductors and insulators. The dominant decay pathway of color centers at moderate temperatures is through photoionization (on irradiation) with the formation of free carriers and empty traps. The active state of a photocatalytic center of a certain sort can be described as a surface color center.

Note that the surface $\mathbf{F}$ and $\mathbf{V}$ centers in semiconductors and insulators are also color centers with the distinction that they are metastable active states of photocatalytic centers.

\section{CONDUCTION BAND}

A vacant or only partially occupied set of many closely spaced electronic levels resulting from an array of a large number of atoms forming a solid system in which electrons can move freely or nearly so. The term is usually used to describe the electrical properties (among several others) of metals, semiconductors and insulators.

\section{CONVERSION SPECTRUM}

A plot of a quantity related to the absorption ( $a b$ sorbance, cross section, etc...) multiplied by the quantum yield for the process considered against a suitable measure of photon energy, such as frequency, $v$, wavenumber, $v^{\prime}$, or wavelength, $\lambda$. 
See also action spectrum, spectral photon effectiveness.

\section{CUT-OFF FILTER}

An optical device that only permits the transmission of radiation of wavelengths that are longer or shorter than a specified wavelength. Usually, the term refers to devices that transmit radiation of wavelengths longer than the specified wavelength.

See filter.

\section{DECAY OF THE ACTIVE STATE OF A PHOTOCATALYTIC (OR PHOTOADSORPTION) CENTER}

For solid photocatalysts, the decay occurs by any physical pathway that leads the photocatalytic (or photoadsorption) center from its active state to its inactive state.

Note If the active state of a given photocatalytic center is an electronically excited state, then the pathways are mainly radiative and non-radiative transitions into lower energy states of the center. However, if the active state of the photocatalytic center is formed by photocarrier trapping, its deactivation may then occur by thermoionization, by photoionization, by recombination with free carriers of the proper sign, or by tunneling recombination with adjacent trapped carriers of the proper sign. Thus, an active state of a photocatalytic center can be described (as in solid-state physics) as either a shallow trap, or as a deep trap. The latter in turn can be a recombination center or a color center depending on the dominant decay pathway.

See also lifetime of an active state of a photocatalytic center.

\section{DEEP ENERGY TRAP}

The trap defined by an energy level (of trapped carriers) within the bandgap of a semiconductor or insulator whose energy difference between it and the conduction band (for electrons) or valence band (for holes) is much greater than the energy of thermal excitation of the solid at a given temperature. The dominant decay pathway of the trap is trapping of carriers of the opposite sign (see recombination center) or photoionization (see color center).

Note that the active state of a photocatalytic center of a certain sort can be described as a deep trap (as done in solid-state physics).

\section{DEPTH OF PENETRATION (of light)}

The term refers to the inverse of the absorption coefficient. The SI unit is $\mathrm{m}$. Sometimes the unit $\mathrm{cm}$ is also used. If the decadic absorption coefficient, $a$, is used, the depth of penetration $(1 / a)$ is taken as the distance at which the spectral radiant power, $P_{\lambda}$, decreases to a tenth of its incident value, $P_{\lambda}^{o}$. If the Napierian absorption coefficient, $\alpha$, is used, the depth of penetration $(1 / \alpha=\beta$ in this case) is taken as the distance at which the spectral radiant power decreases to 1 /e of its incident value.
In semiconductor photochemistry, it is the inverse of the absorption coefficient $(1 / \alpha)$, which determines the space charge region within which photocarriers are generated in a solid (photocatalyst) particle.

See absorbance, attenuance.

\section{DEPLETION LAYER}

The situation that occurs when the surface layer of a (in this case $n$-type) semiconductor becomes depleted of majority carriers (electrons) leading to upward band bending.

As well, it is the sub-surface space wherein the surface charge, whose sign is identical to the sign of the majority charge carriers, in the semiconductor leads to depletion of these carriers.

See accumulation layer, band bending, and inversion layer.

\section{DIELECTRICS (insulators)}

Materials whose electrical conductivity is far less than either a metal or a semiconductor.

Note that in intrinsic semiconductors the generation of carriers occurs because of the thermal excitation of the material. Thus, the bandgap energy in such materials must be comparable to the thermal excitation energy, kT. Taking into account the very high density of electronic states in the valence band, a significant concentration of carriers can be achieved in materials with bandgaps up to $2 \mathrm{eV}$. In this regard, $\mathrm{TiO}_{2}$ and $\mathrm{ZnO}$ with bandgap energies of $c a .3 \mathrm{eV}$ should in fact behave as dielectrics. However, because of the presence of intrinsic defects, such as interstitial metal cations considered as shallow traps, they turn into $n$-type semiconductors because of the thermal ionization of those defects. Moreover, in any material with a bandgap ca. $3 \mathrm{eV}$, all the defects will be thermally ionized since the deepest energy defect is at a depth of about $1.5 \mathrm{eV}$ that can be covered by thermal excitation. Thus, the mechanism of thermoproduction of carriers in such materials is rather different compared to "true" semiconductors.

\section{DIFFUSE LAYER}

The layers in which, because of thermal agitation, the nonspecifically adsorbed ions or molecules are distributed in a three-dimensional region. This layer extends from the outer Helmholtz plane into the bulk of the solution.

\section{DIRECT BANDGAP SEMICONDUCTOR}

Semiconductor or dielectric materials whose first band-to-band transition occurs from the highest level in the valence band to the lowest level in the conduction band possessing the same electron momentum, that is $\mathbf{k}_{\max }=\mathbf{k}_{\min }$, where $\mathbf{k}_{\max }$ and $\mathbf{k}_{\min }$ are the momentum vectors of electrons of the highest level in the valence band and the lowest level in the conduction band, respectively. When the semiconductor (or insulator) is photoexcited, electrons change their energy state owing to the absorption of photons and maintain the same momentum. 
See indirect bandgap semiconductor.

DOPING (a semiconductor)

A process to increase the electrical conductivity of a semiconductor by addition of certain impurities in small quantities (ppm).

\section{DosE}

The energy (in Joules) or amount of photons (in mols) absorbed per unit area or unit volume by an object irradiated during a given exposure time.

In medicine and in some other research areas, the term dose is used in the sense of fluence, that is the energy or amount of photons per unit area or unit volume received by an irradiated object during a particular exposure time. The SI units are $\mathrm{J} \mathrm{m}^{-2}$ or $\mathrm{J} \mathrm{m}^{-3}$, and mol $\mathrm{m}^{-2}$ or $\mathrm{mol} \mathrm{m} \mathrm{m}^{-3}$, respectively.

See also $U V$-dose, and radiation dose.

\section{DRIVING FORCE (for reaction)}

Term widely used to indicate the thermodynamic allowability of a reaction or process to occur as expressed by the negative of the Gibbs free energy change $\left(\Delta G^{\circ}\right)$ for that reaction or process.

\section{DYE SENSITIZATION (of a semiconductor)}

The process in which the dye absorbs light energy to yield an excited state, which in turn transfers an electron (or energy) onto the semiconductor.

See sensitization.

\section{DYNAMIC PHOTOADSORPTION PROCESS}

The simultaneous (or synchronous) formation of adsorbed species via photoadsorption of molecules (or atoms or ions-so-called adsorbates) and their destruction by photodesorption to yield the same adsorbate molecules (or atoms or ions), with both processes occurring under photoexcitation of the solid photocatalyst (adsorbent).

\section{EFFECTIVE RADIOCATALYTIC ACTIVITY OF A SYSTEM}

The number of product molecules generated by each $100 \mathrm{eV}$ of energy of radiation incident on the system.

\section{EFFICIENCY (of a step, $\eta$ )}

Traditionally, it refers to the ratio between the useful energy delivered or bound versus the energy supplied, i.e., energy output/energy input.

In photochemistry and elsewhere, it is a quantitative measure of the relative rate of a given step involving a species (or excited state) with respect to the sum of rates of all of the parallel steps which depopulate that species (or excited state).

See also quantum yield.

\section{ELECTRICAL DOUBLE LAYER}

The whole array of charged species and oriented dipoles existing at a semiconductor-solution (or metalsolution) interface. Note that the solution side consists of several "layers".

See inner layer, diffuse layer.

\section{ELECTRONIC ENERGY MIGRATION (or hopping)}

The movement of electronic excitation energy from one molecular entity to another entity of the same species, or from one part of a molecular entity to another of the same kind (e.g., excitation migration between chromophores of an aromatic polymer). The migration may occur by radiative or nonradiative (radiationless) processes.

\section{ELECTRON TRANSFER PHOTOSENSITIZATION}

A photochemical process in which a reaction of a non-absorbing substrate is induced by electron or energy transfer from an excited light-absorbing substrate that acts as the sensitizer. The overall process must be such that the sensitizer is recycled. Depending on the action of the excited sensitizer as either an electron donor or acceptor during the sensitization, the process is called reductive or oxidative, respectively.

See also photosensitization.

\section{EMISSION}

Radiative deactivation of an excited species or state by transfer of energy from an entity (molecular or otherwise) to an electromagnetic field.

See also fluorescence, luminescence, and phosphorescence.

\section{EMISSION SPECTRUM}

Plot of the emitted spectral radiant power (spectral radiant excitance) or of the emitted spectral photon irradiance (spectral photon excitance) against a quantity related to photon energy, (frequency, $v$, wavenumber, $v^{\prime}$, or wavelength, $\lambda$ ). When corrected for wavelengthdependent variations in the equipment response, it is referred to as a corrected emission spectrum.

\section{EMITTANCE}

See radiant excitance.

\section{ENERGY STORAGE EFFICIENCY}

The rate at which the Gibbs energy is stored in an endothermic photochemical reaction divided by the incident irradiance.

See also efficiency.

\section{ENERGY TRANSFER}

From a phenomenological point of view, the term describes the process by which an entity (molecular or otherwise) absorbs light followed by a phenomenon ocurring from the excited state of another entity (molecular or otherwise). In mechanistic photochemistry, the term is used to describe the photophysical process in which an excited state of one entity (the donor) is deactivated to a lower-lying state by transferring energy to a second entity (the acceptor), which is thereby raised to a higher energy state. The excitation may be electronic, vibrational or translational. Where the donor and acceptor are two parts of the same molecular entity, the process is called intramolecular energy transfer.

See also radiative energy transfer. 


\section{EXCITATION SPECTRUM}

Plot of the spectral radiant excitance or of the spectral photon excitance against frequency (or wavenumber, or wavelength) of excitation. When corrected for wavelength-dependent variations in the excitation radiant power it is referred to as a corrected excitation spectrum.

See also emission spectrum.

\section{EXCITED STATE}

A state of higher energy than the ground state of a chemical entity. In photochemistry one usually means an electronically excited state.

In heterogeneous photocatalysis, photogenerated free electrons in the conduction band and free photogenerated holes in the valence band are in essence excited states of the photocatalyst. Free and bound $e x$ citons are also a type of excited states of photocatalysts. The photocatalyst with a photocarrier trapped by bulk and surface defects, even in the absence of free photocarriers at the end of irradiation, is in an excited metastable state in relation to the ground state of the solid particle without a trapped photocarrier. As in the case of free photocarriers, the existence of trapped photoelectrons and photoholes also leads to Fermi level splitting. Thus, the active state of a photocatalytic (photoadsorption) center is a particular case of an excited state of the photocatalyst. At the same time, the active state of the photocatalytic center with a trapped carrier can be in its lowest energy state (typically) in relation to the set of possible electronic states in the solid subsystem (empty defect; carrier).

\section{EXCITON}

A bound electron-hole pair (neutral quasi-particle, electronic excitation) in semiconductors and insulators capable of migrating and transferring its energy in the solid lattice (free exciton). An exciton trapped by a defect (localized exciton) is identical to the electronically excited state of the defect.

In other applications it is useful to consider electronic excitation as a quasi-particle also capable of migrating. For example, in organic materials two models are used: the band or wave model (low temperature, high crystalline order) and the hopping model (higher temperature, low crystalline order or amorphous state). In the hopping limit, energy transfer is identical with energy migration.

See electronic energy migration.

\section{EXCITON ABSORPTION}

Absorption of light yielding optical information on the excitons in solids. Exciton absorption bands in an absorption spectrum of a semiconductor or insulator are located on the long wavelength side of the fundamental absorption edge.

EXTRINSIC ABSORPTION (of solid photocatalysts)

The absorption of light in semiconductors and insulators corresponding to optical transitions of electrons from defect energy levels (within the bandgap) to conduction band levels, or of holes from defect levels to valence band levels (photoionization of defects); or from the ground state level to excited levels of a given intrinsic defect of any sort. Typically, extrinsic absorption bands are a few tenths to a few eV distant from the fundamental absorption edge in semiconductors and insulators. The red limits of photocatalytic reactions are in fact determined by photoexcitation of photocatalysts in the extrinsic absorption bands.

\section{EXTRINSIC EMISSION}

Luminescence obtained from optical transitions between intra-bandgap energy levels of extrinsic or intrinsic defects and the conduction and valence band levels of the semiconductor or insulator.

\section{EXTRINSIC SURFACE DEFECTS}

Imperfections in a solid semiconductor (or insulator) connected with surface defects of any sort involving extrinsic chemical units in relation to the chemical formula of the solid, particularly adsorbed species of any sort.

These extrinsic surface defects play an essential role in heterogeneous photocatalysis. For example, they can serve as photocatalytic centers (sites). Surface defects, as well as bulk defects located in the near-surface region can serve as entities that absorb light and generate free charge carriers. Surface defects can trap charge carriers and serve as recombination centers, thereby influencing the concentration of photocarriers at the surface of photocatalyst particles.

\section{FERMi LEVEL $\left(E_{\mathrm{F}}\right)$}

The chemical potential of electrons in a solid (metal, semiconductors or insulators) or in an electrolyte solution.

In irradiated semiconductors and insulators, the Fermi level splits into two Fermi quasi-levels for electrons $\left(E_{\mathrm{F}}{ }^{\mathrm{e}}\right)$ and for holes $\left(E_{\mathrm{F}}{ }^{\mathrm{h}}\right)$ that are displaced toward the bottom of the conduction band and toward the top of the valence band, respectively. The greater the $r a$ diant power of the actinic light is, the greater is the Fermi level splitting: $\Delta F=E_{\mathrm{F}}^{\mathrm{e}}-E_{\mathrm{F}}{ }^{\mathrm{h}}$. The positions of the Fermi quasi-levels within the bandgap (typically) determine the concentration of free thermalyzed photocarriers in irradiated solid photocatalysts.

See bandgap energy, conduction band, valence band.

\section{FILTER (optical)}

A device that reduces the spectral range (bandpass, cut-off, and interference filter) or radiant power of incident radiation (neutral density or attenuance filter) upon transmission of radiation.

\section{FLATBAND POTENTIAL}

The potential at which no excess charge exists in the semiconductor (potential of zero charge), and there is 
no electric field and no space charge region so that the (conduction and valence) bands are not bent.

\section{FLUENCE $\left(H_{0}\right)$}

When applied to energy, it is the total radiant energy traversing a small transparent imaginary spherical target containing the point under consideration divided by the cross section of this target. It is also the product of the fluence rate $\left(E_{0}\right)$ and the duration of the irradiation ( $\int E_{0} \mathrm{~d} t$, simplified expression: $H_{0}=E_{0} t$ when the fluence rate is constant over the time considered). The SI unit is $\mathrm{J} \mathrm{m}^{-2}$. Energy fluence is identical to spherical radiant exposure and reduces to radiant exposure $(H)$ for a parallel and normally incident beam, that is neither scattered nor reflected by the target or its surroundings.

See also dose, photon fluence.

FLUENCE RATE $\left(E_{0}\right)$

It is the rate of fluence $\left(H_{0}\right)$ and four times the ratio of the radiant power $(P)$ incident on a small transparent imaginary spherical volume element containing the point under consideration, divided by the surface area of that sphere $\left(S_{\mathrm{K}}\right) ;\left(\int_{4 \pi} L \mathrm{~d} \omega\right.$ simplified expression: $E_{0}=4 P / S_{\mathrm{K}}$ when the radiant power is constant over the solid angle considered). For the energy fluence rate, the SI unit is $\mathrm{W} \mathrm{m}^{-2}$. It reduces to irradiance, $E$, for a parallel and perpendicular incident beam that is neither scattered nor reflected by the target or its surroundings.

See intensity, radiance.

See also photon fluence rate.

\section{FLUORESCENCE}

Emission of radiation from an excited state of a given multiplicity to the ground state of the same multiplicity (e.g., from a singlet excited state to a singlet ground state).

\section{FLUX (energy)}

See radiant (energy) flux, radiant power.

\section{FREQUENCY $(v$ or $\omega)$}

Refers to the number of wave periods per unit time. The linear frequency, $v$, is the number of cycles per unit time. The SI unit is $\mathrm{Hz}=\mathrm{s}^{-1}$. For the angular frequency, the symbol $\omega(=2 \pi v)$ is used, with $\operatorname{rad~s}^{-1}$ as the SI unit.

\section{FUNDAMENTAL ABSORPTION (of solid photocatalysts)}

Absorption of light in semiconductors and insulators corresponding to optical transitions of electrons from the valence band to the conduction band to yield free electron-hole pairs and/or exciton absorption bands.

See also intrinsic absorption, extrinsic absorption, exciton absorption.

FUNDAMENTAL ABSORPTION EDGE (threshold - of solid photocatalysts)

The high wavelength limit of the fundamental $a b$ - sorption band in the absorption spectrum of semiconductors and insulators.

\section{GROUND STATE}

The lowest energy state of a chemical entity. In photochemistry, it refers to the ground electronic state.

\section{HETEROGENEOUS PHOTOCATALYSIS}

Photocatalysis taking place at the interfacial boundary between two phases (solid-liquid; solid-gas; liquidgas).

See photocatalysis.

\section{HETEROGENEOUS RADIOCATALYSIS}

Radiation catalysis taking place in a heterogeneous system.

See radiocatalysis.

\section{HOLE TRANSFER}

Refers to the migration of charge in a solid (metals, semiconductors, insulators) in which the majority carrier is positively charged.

\section{HOMOGENEOUS PHOTOCATALYSIS}

Photocatalysis taking place in a homogeneous phase.

\section{HOMOGENEOUS RADIOCATALYSIS}

Radiation catalysis taking place in a homogeneous phase.

\section{HYPSOCHROMIC SHIFT}

Refers to the shift of a spectral band to a higher frequency (or shorter wavelength) upon changing a substituent in a molecular entity or a change in medium (e.g., solvent). Informally, it is referred to as a blue shift, and is the opposite of a bathochromic shift.

\section{INDIRECT BANDGAP SEMICONDUCTOR}

Semiconductor or dielectric materials whose first band-to-band transition from the highest level in the valence band to the lowest level in the conduction band requires a change of electron momentum, that is $\mathbf{k}_{\text {max }} \neq \mathbf{k}_{\text {min }}$, where $\mathbf{k}_{\text {max }}$ and $\mathbf{k}_{\text {min }}$ are the momentum vectors of electrons of the highest level in the valence band and the lowest level in the conduction band, respectively. When the semiconductor (or insulator) is photoexcited such changes occur owing to interaction of the electronic subsystem with the phonons. Direct band-to-band electronic transitions are forbidden because of a change in momentum.

See direct bandgap semiconductor

\section{INNER FILTER EFFECT}

This term is used in two different ways. In an emission experiment, it refers to a seeming decrease in emission quantum yield and/or distortion of bandshape as a result of reabsorption of emitted radiation. During a light irradiation experiment, absorption of incident radiation by a species other than the intended primary absorber is also described as an inner filter effect. 


\section{INNER HELMHOLTZ PLANE}

The locus of electrical centers of specifically adsorbed ions.

See outer Helmholtz plane.

\section{INNER LAYER}

The layer closest to the semiconductor surface (in an electrical double layer) that contains solvent molecules and sometimes other species (ions or molecules) that are specifically adsorbed. This layer is often referred to as a compact layer, Helmholtz layer, or Stern layer.

See diffuse layer.

\section{INTEGRATING SPHERE}

A hollow spherical device having a highly reflecting inner surface. It is used to collect, with very high efficiency, light scattered or emitted from a sample contained inside it or located outside but near one of its ports. The small ports allow the entrance of light and facilitate access to the detector.

\section{INTENSITY}

Traditionally, this term has been used to refer to (confusingly) photon flux, fluence rate, irradiance and radiant power (radiant flux). For an object exposed to radiation, the term should be used only for qualitative descriptions.

Intensity (of a light source; $I$ ) is the same as radiant intensity.

Intensity (of a spectral feature) refers to the magnitude of that particular feature in the spectrum (absorption, emission, etc...).

\section{INTERPARTICLE ELECTRON TRANSFER (IPET)}

Electron transfer that takes place from a photoexcited semiconductor onto another semiconductor (or insulator) in its ground state leading to vectorial (irreversible) charge transfer.

\section{INTRINSIC ABSORPTION}

See fundamental absorption.

\section{INTRINSIC EMISSION}

Band-to-band recombination of electrons and holes (or exciton decay) in a semiconductor or insulator leads to luminescence.

\section{INTRINSIC SURFACE DEFECTS}

Imperfections connected with surface point defects (zero-dimensional), linear defects (one-dimensional) or square defects (two-dimensional) that do not include extrinsic chemical units in relation to the chemical formula of the solid.

Surface vacancies, interstitial lattice atoms and ions, edges, corners, and kinks are some examples of intrinsic surface defects.

\section{INVERSION LAYER}

Layer formed when band bending creates the situation in which the Fermi level on the surface is placed close to the band of the bulk minority charge carriers. This leads to a higher concentration of minority charge carriers on the surface relative to the concentration of the majority charge carriers, a situation that is opposite to that in the bulk.

See accumulation layer and depletion layer.

\section{IRRADIANCE $(E)$}

The radiant flux or radiant power $(P)$ of all wavelengths incident on an infinitesimal element of surface, containing the point under consideration, divided by the area of the element $(\mathrm{d} P / \mathrm{d} S$, simplified expression: $E=P / S$ when the radiant power is constant over the surface area considered). The SI unit is $\mathrm{W} \mathrm{m}^{-2}$.

Note that $E=\int E_{\lambda} \mathrm{d} \lambda$, where $E_{\lambda}$ is the spectral irradiance at wavelength $\lambda$. For a parallel and perpendicularly incident beam that is neither scattered nor reflected by the target or its surroundings, fluence rate $\left(E_{0}\right)$ is an equivalent term.

See also photon irradiance, spectral irradiance.

\section{ISOSBESTIC POINT}

Refers to a wavelength (or wavenumber or frequency) at which the total absorbance of a sample does not change during a chemical reaction or physical change of the sample. The term originated from the Greek word for: "same attenuance". As a simple example, consider a molecular entity which is converted into another, and both of which have the same molar absorption coefficient at the given wavelength. As long as the sum of the concentrations of the two molecular entities in the solution remains constant there will be no change in absorbance at this wavelength, even though the ratio of the two entities may vary. In general, $A_{\lambda} l^{-1}\left(=\Sigma_{n}{ }^{i=1} \varepsilon_{i}{ }^{\lambda} c_{i}\right)$ must remain constant during the reaction or physical change in order to observe an isosbestic point. The sometimes-used term isoabsorption point should not be used.

\section{LAMBERT LAW}

Describes the fraction of light absorbed by a system that is independent of the incident spectral radiant power $\left(P_{\lambda}^{o}\right)$. This law holds only if $P_{\lambda}^{o}$ is small, scattering is negligible, and multiphoton processes, excited state populations, and photochemical reactions are negligible.

See absorbance, and Beer-Lambert law.

\section{LAMP}

A device that is a source of incoherent radiation.

\section{LANGMUIR-HINSHELWOOD KINETICS}

In photocatalytic (or photoadsorption) reactions, the rate dependence on reagent concentration (pressure for gases) that can be approximated by the equation:

$$
r=-\frac{\mathrm{d} C}{\mathrm{~d} t}=\frac{k \mathrm{~K} C}{(1+\mathrm{K} C)}
$$

where $k$ and $\mathrm{K}$ are the apparent rate constants and adsorption coefficients, respectively, obtained at a given 
radiant power of the actinic light, and $C$ (or $p$ ) is the reactant concentration (or pressure).

Note that observation of Langmuir-Hinshelwood kinetics does not infer that the Langmuir-Hinshelwood mechanism is operative. For example, the alternative Eley-Rideal mechanism yields the same types of kinetics provided that the active state of the surface catalytic (photoadsorption) center has a limited lifetime. Moreover, even if the Langmuir-Hinshelwood mechanism were opperational, both constants, $k$ and $\mathrm{K}$, can be the effective parameters and need not correspond to the elementary reaction rate constant and Langmuir adsorption constant, respectively. Consequently, the experimental dependence obeying eq. (49) cannot be considered a reasonable motive to infer a certain mechanism.

\section{LIFETIME $(\tau)$}

The lifetime of an entity that decays by a (pseudo) first-order process is the time needed for the concentration of the entity to decrease to 1/e of its original value. Statistically, it represents the life expectation of the entity. It is equal to the reciprocal of the sum of the (pseudo)-unimolecular rate constants of all the processes responsible for the decay.

Lifetime is sometimes also used for processes that are not first-order. In such cases the lifetime depends on the initial concentration of the entity (or of the quencher) and on the light irradiance. Consequently, only an initial or mean lifetime can be defined. In this case, the terms initial lifetime or mean lifetime should be specified.

Occasionally, the term half-life $\left(\tau_{1 / 2}\right)$ is used to represent the time needed for the concentration of an entity to decrease to one half of its original value.

LIFETIME OF AN ACTIVE STATE OF A PHOTOCATALYTIC (OR PHOTOADSORPTION) CENTER $\left(\tau_{c}\right)$

The reciprocal of the sum of all the decay constants (typically pseudo first-order constants) corresponding to all the possible physical processes that lead to the transformation of a photocatalytic (or photoadsorption) center from its active state to an inactive state. The decay of the active state of the photocatalytic center caused by a chemical process (reaction channel) is not considered in this context because this channel is typically much slower than the physical channels.

An estimate of $\tau_{c}$ can be obtained from LangmuirHinshelwood kinetics. For example, for a reaction such as

$$
\mathrm{A}+\mathrm{C} \underset{k_{2}}{\stackrel{k_{1}}{\gtrless}}(\mathrm{A}-\mathrm{C}) \stackrel{k_{3}}{\longrightarrow} \text { products }
$$

$\tau_{c} \geq 1 / k_{2}$, where $k_{2}$ is the pseudo first-order rate constant defined by the ratio $\mathrm{K}=k_{1} / k_{2}, \mathrm{~K}$ is the pseudo Langmuir adsorption constant and $k_{1}$ is the secondorder rate constant related to the interaction of the adsorbate (A, or reactant molecule) with the photoadsorp- tion (photocatalytic) center C. If $k_{1}$ is taken as the collision rate constant, then the estimate of $\tau_{\mathcal{C}}$ is possible.

\section{LIGHT INITIATED THERMOCATALYTIC PHENOMENA} See thermophotocatalysis.

\section{LUMINESCENCE}

Spontaneous emission of radiation from an electronically or vibrationally excited species not in thermal equilibrium with its environment.

\section{LUMOPHORE}

Refers to a part of an entity (atom or group of atoms) in which electronic excitation associated with a given emission band is approximately localized (analogous to chromophore for absorption spectra).

\section{MASS TRANSFER}

Movement of material from one location in solution to another as might arise either from differences in electrical or chemical potential at the two locations, or from movement of a volume element of solution. This notion is particularly significant in heterogeneous photocatalysis when the photocatalyst is anchored (i.e., immobilized) on a support, and the resulting kinetics of the photoprocess may be mass-transfer limited.

MAXIMAL PHOTOADSORPTION CAPACITY (of an adsorbent, $\left.\theta_{\mathrm{m}}\right)$

The maximal number of molecules (atoms, ions) per regular surface site that can be photoadsorbed at a uniformly irradiated surface of a given adsorbent. Note that $\theta_{\mathrm{m}}$ is a dimensionless quantity.

$$
\theta_{\mathrm{m}}=\frac{N_{\mathrm{m}}}{N_{\mathrm{ss}}}
$$

where $N_{\mathrm{m}}$ is the maximal number of molecules photoadsorbed and $N_{\mathrm{ss}}$ is the number of surface sites (mainly regular surface sites, i.e., regular lattice ions or atoms; typically, $N_{\mathrm{ss}} \sim 10^{15} \mathrm{~cm}^{-2}$ ). The quantity $N_{\mathrm{m}}$ can be obtained by approximation from photoadsorption kinetics $N(t)$ on a uniformly irradiated surface.

(i) If the powdered sample is uniformly irradiated, then the area of the irradiated surface $S=m \sigma$, where $m$ is the mass of the sample [units: $g$ ] and $\sigma$ is the specific surface area [units: $\mathrm{cm}^{2} / \mathrm{g}$ ] of a given adsorbent.

(ii) If photoadsorption is the stage of a given photocatalyzed reaction and if the efficiency of desorption of the adsorbed species (via a physical process) is negligible, then the number of photocatalytic centers can be estimated from the maximal photoadsorption capacity, $\theta_{\mathrm{m}}$, to determine TON and TOF for a given photocatalytic reaction. Known experimental values of $\theta_{\mathrm{m}}$ fall in the range $10^{10}$ to $10^{12} \mathrm{~cm}^{-2}$.

See turnover number, turnover frequency, dynamic photoadsorption process and photoadsorption capacity. 
MAXIMAL PHOTOADSORPTION STOICHIOMETRY (of an adsorbent)

Maximal number of molecules (atoms, ions) that can be photoadsorbed per regular surface site on a uniformly irradiated surface of a given adsorbent.

See also photoadsorption capacity.

MEMORY EFFECT (Coekelbergs-Solonytsin effect)

The post-irradiation adsorption (post-adsorption) event at the surface of a pre-irradiated adsorbent (photocatalyst) occurring in the dark after irradiation is terminated.

The Coekelbergs effect is an effect of continuing post-irradiation adsorption after the termination of irradiation in the presence of photoadsorbate molecules; that is, after preliminary photoadsorption.

The Solonytsin effect refers to a post-irradiation adsorption after termination of irradiation in vacuo.

The memory effect can be characterized quantitatively by the post-adsorption memory coefficient, K:

$$
\mathrm{K}(t)=\frac{N_{\text {post }}(t)}{N_{\text {phot }}(t)}
$$

where $N_{\text {post }}(t)$ is the number of molecules adsorbed after termination of irradiation for a given time, $t$, and $N_{\text {phot }}(t)$ is the number of photoadsorbed molecules during the same time of irradiation, $t$. Typically, the value of the memory coefficient $\mathrm{K}$ is a function of the time of pre-irradiation.

\section{MINERALIZATION}

The process by which an organic substrate is converted into carbon dioxide and water, etc....

MOLAR ABSORPTION COEFFICIENT (decadic, $\varepsilon$; Napierian, $\kappa$ )

Absorbance divided by the absorption pathlength, $l$, and the concentration, $c$ :

$$
\varepsilon=\frac{a}{c}=\left(\frac{1}{l c}\right) \log \left(\frac{P_{\lambda}^{o}}{P_{\lambda}}\right)=\frac{A}{l c}
$$

and

$$
\mathrm{K}=\frac{\alpha}{c}=\left(\frac{1}{l c}\right) \ln \left(\frac{P_{\lambda}^{o}}{P_{\lambda}}\right)=\frac{A}{l c}
$$

In common usage, $l$ has units of $\mathrm{cm}$ and $c$ of mol $\mathrm{dm}^{-3}$ (or $\mathrm{M}$ ), $\varepsilon$ results in $\mathrm{dm}^{3} \mathrm{~mol}^{-1} \mathrm{~cm}^{-1}$ or $\mathrm{M}^{-1} \mathrm{~cm}^{-1}$.

The use of the term molar absorptivity for the molar absorption coefficient should be avoided.

See also absorbance, absorption coefficient, and BeerLambert law.

\section{NATURAL PHOTOSYNTHESIS}

The production of complex organic substances by superior plants, algae and photosynthesizing bacteria from simple compounds (e.g., carbon dioxide and water) at the cost of light energy absorbed by chlorophyll and other photosynthesizing pigments.
See also artificial photosynthesis.

NEAR-SURFACE REGION (of a solid particle)

The region or layer just below the surface of the solid particle a few lattice constants deep that is typically perturbed by the surface, by surface defects, and surface electric fields.

Note by "perturbation" we refer to the consequences of the bulk translational symmetry violation in a crystal caused by such surface events as (i) shift of regular atoms and ions, i.e., relaxation and reconstruction, (ii) space redistribution of the concentration of defects compared with that in the bulk of the crystal lattice, (iii) the appearance of a space charge layer connected with surface charge, etc....

\section{NON-LINEAR OPTICAL EFFECT}

An effect brought about by electromagnetic radiation the magnitude of which is not proportional to the irradiance. Some important non-linear optical effects are: harmonic frequency generation, lasers, Raman shifting, upconversion, and others.

NON-RADIATIVE DECAY (or radiationless decay)

Disappearance of an excited species due to a radiationless transition.

\section{OUTER Helmholtz PLANE}

The locus of centers of nearest solvated ions that are nonspecifically adsorbed.

See inner Helmholtz plane.

\section{PHONON}

Elementary excitation in the quantum mechanical treatment of vibrations in a crystal lattice.

\section{PhOSPHORESCENCE}

From a phenomenological point of view, the term has been used to describe long-lived luminescence. In mechanistic photochemistry, the term designates luminescence involving two states of different spin multiplicity (e.g., from triplet to singlet or vice versa, and from a quartet state to a doublet state). In solidstate physics, phosphoresence is connected to the thermal detrapping of carriers from the shallow traps followed by the radiative trapping of carriers by the emission centers (deep traps). In this context, the term phosphorescence reflects the longer decay of excitation after irradiation of the solids has been terminated.

Photoadsorbate (photoadsorbed species)

A molecule (or atom, or ion) bound to the surface of a solid (semiconductor or insulator) particle (typically to a photoadsorption center) as the result of photexcitation. If photoadsorption is a step in a photocatalytic reaction, the photoadsorbed species may be considered an intermediate in the given reaction.

\section{PhotoAdsorption (photoinduced adsorption)}

Adsorption, typically chemisorption (i.e., a stoichiometric reaction of adsorbate molecules with a 
solid surface), initiated by light absorbed either by the adsorbate or by the adsorbent. Often it is considered as a chemical step (primary) in a heterogeneous photo catalytic reaction.

PhotoAdsorption AREAL CAPACITY (of an adsorbent; $\theta_{A}$ )

The amount of molecules (or atoms, or ions) photoadsorbed per unit area on the uniformly irradiated surface of a given adsorbent.

See also maximal photoadsorption capacity.

PHOTOADSORPTION CAPACITY (of an adsorbent; $\theta$ )

A dimensionless quantity referring to the number of molecules (atoms, ions) photoadsorbed per regular surface site at a uniformly irradiated surface of a given adsorbent.

See maximal photoadsorption capacity.

\section{PHOTOADSORPTION CENTER}

A surface site or a surface defect in its active state after photoexcitation capable of forming photoadsorbed species by a chemical interaction with adsorbate molecules (or atoms, or ions).

See also active state of a surface photoadsorption center.

\section{PHOTOADSORPTION EFFICIENCY (PAE) $\lambda$}

The ratio of the maximal (typically initial) rate of photoadsorption to the incident photon flow at a given wavelength $\lambda$. Both rates are measured under otherwise identical conditions and are expressed in the same units in order to avoid concentration and/or surface area effects.

$$
(\mathrm{PAE})_{\lambda}=\frac{\mathrm{d} N_{\mathrm{mol}} / \mathrm{d} t}{\mathrm{~d} N_{\mathrm{ph}} / \mathrm{d} t}
$$

where $\mathrm{d} N_{\mathrm{mol}} / \mathrm{d} t$ is typically the number of molecules (corresponding to the initial quasi-stationary rate of photoadsorption) adsorbed per unit time (seconds) and $\mathrm{d} N_{\mathrm{ph}} / \mathrm{d} t$ is the number of incident photons also per unit time.

The initial rate of photoadsorption can also be presented as $\mathrm{d} p / \mathrm{d} t\left(\mathrm{~Pa} \mathrm{~s}^{-1}\right)$ for a gas/solid system, or as $\mathrm{d} C / \mathrm{d} t$ for liquid/solid systems.

Note that the $(\mathrm{PAE})_{\lambda}$ depends on the concentration (pressure) and on the photon flow. If the photoadsorption efficiency is determined under first-order conditions in photon flow and zero-order on concentration (pressure), its spectral dependence $(\mathrm{PAE})_{\lambda}$ will be similar (typically equal) to the efficiency of formation of the active photoadsorption center.

\section{PHOTOADSORPTION QUANTUM YIELD}

The ratio of the number of photoadsorbed molecules, atoms, ions, radicals or photoadsorbed species on a surface to the number of photons of light (at a given wavelength $\lambda$ ) absorbed by the system (typically by the adsorbent) during the initial stage of irradiation.
Alternatively, it can be expressed as the rate of photoadsorption divided by the rate of photons absorbed (in identical units).

\section{Photoassisted catalysis}

See catalyzed photolysis and photocatalysis.

\section{Photobleaching}

In photochemistry this term refers to the loss of absorption or emission intensity occurring as a result of absorption of light quanta by the substrate. In semiconductor photochemistry and photophysics, it refers to the loss of color (loss of absorption) by the color centers as a result of absorption of light quanta by the solid semiconductor or insulator.

See thermionization.

PHOTOCARRIER (photo charge carrier)

Non-equilibrium free electron or free hole generated in a solid by light absorption and occupyiong energy levels in the conduction band or the valence band, respectively. In photocatalytic mechanisms, photo-electrons occupying energy levels near the bottom of the conduction band, and photo-holes occupying energy levels near the top of the valence band (thermalized carriers) are considered as quasi-chemical intermediates. In the general case, non-thermalyzed (hot) carriers can also be involved in photocatalyzed reactions.

\section{Photocatalysis}

In its most simplistic description, it denotes the acceleration of a photoreaction by the action of a catalyst.

Note that it also refers to a general label to indicate that light and a substance (the catalyst or initiator) are necessary entities to influence a reaction. The catalyst may accelerate the photoreaction by interacting with the substrate(s) either in its ground state or in its excited state, and/or with the primary product, depending on the mechanism of the photoreaction. Such a description also embraces photosensitization and yet such a process, defined officially as a process whereby a photochemical change occurs in one molecular entity as a result of initial photon absorption by another molecular species, the photosensitizer, is not necessarily catalytic without assessing a turnover quantity and/or the quantum yield. The issue rests entirely on the role of the photons. (An earlier IUPAC document defined it as a catalytic reaction involving light absorption by a catalyst or by a substrate.)

\section{Photocatalyst}

See photocatalysis.

Photocatalytic ACtive Center (photocatalytic active site)

A surface center (i.e., either a surface defect or a regular surface site) at which a chemical transformation takes place after converting the photocatalytic center into its active state via any photophysical process 
(e.g., photoexcitation). Note that the number of photoactive centers is very often difficult to establish particularly on solid surfaces.

When the density of the active centers is unknown, the reaction rates may be related to the $\left[B E T\left(N_{2}\right)\right]$ area with the understanding that the parameter or property of the system, which is being measured and reported, will be a lower limit of the actual value.

Note that the photophysical process may include one or several processes such as direct excitation of the photocatalytic center via absorption of light, via photocarrier trapping, via interaction with excitons, and via energy and/or electron transfer from a photoexcited reagent or intermediate.

\section{Рнотоcatalytic activity (of a system)}

The number of molecules (product) formed in a given photocatalytic process (alternatively the number of molecules of a given reactant disappearing) per photon of light absorbed by the photocatalytic system. Note that this quantity has a well-defined meaning only when the reaction operating conditions are also stated and the results are reported in terms of the initial conditions.

See also quantum yield.

\section{Photocatalytic Center (SITE)}

A surface center (i.e., either a surface defect or a regular surface site) that is in an active or electronically excited state at which a chemical transformation takes place.

\section{Photocatalytic EFficiency (of a system)}

The number of molecules (or product) of a given photocatalytic process formed (alternatively, the number of molecules of a given reactant disappearing) per photon of light of a given energy impinging on the photocatalytic system. Note that the quantity has no direct photochemical meaning. However, it may be relevant in practical applications of photocatalysis where the interest is to assess an engineering efficiency of energy out versus energy in. In this context, the quantity has a well-defined meaning only when the reaction operating conditions are also stated and results are reported in terms of the initial conditions.

See quantum yield.

See also: photonic efficiency.

Photochemical CAPACITy OF A HeTERogeneous PHOTOCATALYST (areal, APC, and volumetric, VPC)

The capacity of a photocatalyst to absorb photons is usually defined as the ratio:

(1) Areal photochemical capacity

$$
=\frac{\text { number of absorbed photons }}{\text { (time) (surface area) }}
$$

The term might best be described as absorbed photon irradiance (einstein $\mathrm{s}^{-1} \mathrm{~m}^{-2}$ ) defined as $E_{\mathrm{p}} f$, where $E_{\mathrm{p}}$ is the incident photon irradiance (einstein $\mathrm{s}^{-1} \mathrm{~m}^{-2}$ ) and $f$ is the fraction of light absorbed by the system from the incident beam. The experimental determination of photon flow absorbed requires determining the number of "absorbed photons" during a given period of time.

(2) Note that absorption of radiation is a phenomenon occurring in a volume element. Consequently, the magnitude to be used is the amount of absorbed photons per unit volume and unit time at a given wavelength. Thus, the more strictly defined photochemical capacity must be defined as:

\section{Volumetric photochemical capacity \\ $=($ absorption coefficient of photocatalyst at a given wavelength) $\times$ (photon irradiance),}

where the absorption coefficient has units of $\mathrm{m}^{-1}$ and the photonic irradiance (or incident photon radiation) has units of mol m $\mathrm{m}^{-2} \mathrm{~s}^{-1}$ or einstein $\mathrm{m}^{-2} \mathrm{~s}^{-1}$. The result of this product is, in general, the volumetric rate of photon absorption.

Note that the intrinsic photochemical capacity of a given heterogeneous photocatalyst is characterized, at least partially, by its absorption coefficient. This is a definition that is independent of the amount of radiation employed in the experiment. Note that when using the photonic irradiance, the results do not depend exclusively on the photocatalyst. A rigorous method for measuring the absorption coefficients of heterogeneous photocatalysts has been reported in ref. [18]. It should be noted that the photochemical capacity of a heterogeneous photocatalyst does not only depend on the ability of the semiconductor to absorb radiation, but also on many other factors, all of which are related to geometrical, physical and chemical properties of the solid and which affect the efficacy of the semiconductor photocatalyst.

\section{PHOTOCHEMICAL REACTION (photoreaction)}

Term generally used to describe a chemical reaction caused by absorption of ultraviolet, visible, or infrared radiation. There are many ground state reactions that have photochemical counterparts such as for example photooxidations, photoreductions, etc....

Note that the role of the electronically excited state formed after light absorption is to be emphasized here, as the latter event also causes system heating, which of necessity would also accelerate the thermal component of the reaction.

\section{PhotochemistRy}

The branch of chemistry concerned with the chemical effects of light (from the far UV to the Infrared).

\section{Photochromism}

A photoinduced transformation of a chemical structure (e.g., of a solution), photochemically and/or 
thermally reversible, that produces a spectral change, typically but not necessarily, of visible color.

\section{Photoconductivity}

The increase in the electrical conductivity resulting from the photoproduction of charge carriers.

\section{PHOTOCURRENT}

Photogeneration and movement of charge between two electrodes in a photovoltaic cell or a photoelectrochemical cell as a result of photoprocesses induced by light absorption.

\section{PHOTOCURRENT YIELD}

The quantum efficiency of charge photogeneration between the two electrodes of a photovoltaic cell or a photoelectrochemical cell.

\section{Photodegradation}

The photochemical transformation of a molecule into lower molecular weight fragments taking place in an oxidation or reduction process. This term is widely used in the oxidative or reductive destruction of pollutants by UV-based processes.

\section{PHOTODESORPTION}

Desorption induced by the absorption of light quanta either by the adsorbate or by the adsorbent. In heterogeneous photocatalysis, photodesorption can be a step in the overall mechanism of a photocatalyzed reaction.

See also: dynamic photoadsorption process.

\section{PHOTODESORPTION CROSS-SECTION ( $\left.\sigma_{\text {phd }}\right)$}

The constant $\sigma_{\text {phd }}$ defined by the equation:

$$
\frac{\mathrm{d} N}{\mathrm{~d} t}=-\sigma_{\mathrm{phd}} N_{a} E_{\mathrm{p}}
$$

where $N_{a}$ is the dimensionless quantity of molecules (atoms or ions) of a given type adsorbed at a uniformly irradiated surface of the solid, $E_{\mathrm{p}}$ is the incident photon irradiance at a given wavelength $\lambda$ (or energy $E$ or frequency $v$ ) [ $E_{\mathrm{p}}$ in units of $\mathrm{cm}^{-2} \mathrm{~s}^{-1}$ or $\mathrm{m}^{-2} \mathrm{~s}^{-1}$ ]. Photodesorption cross-section is related to the photodesorption decay time by $\sigma_{\text {phd }}=1 /\left(E_{\mathrm{p}} \tau_{\mathrm{phd}}\right)$ [units: $\mathrm{m}^{2}$ or $\mathrm{cm}^{2}$.

\section{PHOTODESORPTION DECAY TIME}

The constant $\tau_{\text {phd }}$ defined by the kinetic equation:

$$
\frac{\mathrm{d} N}{\mathrm{~d} t}=C \exp \left[-\frac{t}{\tau_{\mathrm{phd}}}\right]
$$

where $\mathrm{d} N / \mathrm{d} t$ is the rate of photodesorption $(\mathrm{d} N / \mathrm{d} t$ in units of $\mathrm{s}^{-1}$ ) of molecules (or atoms, or ions, or radicals) of a given type, $C$ is a kinetic constant equal to the maximal (typically initial) quasi-stationary rate of photodesorption, and $t=$ time. Photodesorption decay times can be obtained from experimental photodesorp- tion kinetics. They are related to the photodesorption cross-section as $\tau_{\text {phd }}=1 /\left(\sigma_{\mathrm{phd}} E_{\mathrm{p}}\right)$.

See photodesorption cross-section.

PHOTODESORPTION QUANTUM YIELD $\left(\Phi_{\mathrm{des}}\right)$

The number of molecules (atoms or ions or radicals) evolving into the gas or the liquid phase from a surface per photon of light absorbed at a given wavelength by a solid or by an adsorbed entity treated as a surface extrinsic defect (center).

(i) the photodesorption quantum yield $\Phi_{\text {des }}$ is given by,

$$
\Phi_{\mathrm{des}}=\frac{\mathrm{d} N_{\mathrm{des}} / \mathrm{d} t}{\mathrm{~d} N_{\mathrm{ph}} / \mathrm{d} t}=\frac{d N_{\mathrm{des}} / d t}{\mathrm{~A} E_{\mathrm{p}}}
$$

where $\mathrm{A}$ is the absorptance of the solid (system), $E_{\mathrm{p}}$ is the photon irradiance. When photoexcitation of a system reduces to light absorption by pre-adsorbed species (see: extrinsic light absorption) $\Phi_{\text {des }}$ does not depend on the concentration of pre-adsorbed species.

(ii) Photodesorption is the inverse process of photoadsorption. Both processes (or reactions) are induced by light from the same wavelength region (see: dynamic photoadsorption process).

\section{PhOTOELECTRICAL EFFECT}

The ejection of an electron from a solid or a liquid.

\section{Photoelectrocatalysis}

This usually has the same physico-chemical nature as photocatalysis. The role of the photocatalyst in photochemical transformations is played by a photoelectrode, which is often a semiconductor. Photoelectrocatalysis and photocatalysis may be distinguished by the fact that for photoelectrocatalytic transformations it is possible to identify an "electrode and an electrolyte" combination in the system along with a rather explicit, generally macroscopic, electrical circuit for the charge transfer and/or for applying additional electric potential to the system. By contrast, this is not generally so in photocatalysis.

\section{PhotoelectrochemicAl CELL}

An electrochemical cell in which current and a voltage are simultaneously produced upon absorption of light by one or more of the electrodes. Usually at least one of the electrodes is a semiconductor.

\section{PhotoelectrochemicAl ETCHING}

The dissolution of a semiconductor in an electrolytic solution on exposure to light. Typically used in the photopatterning of semiconductor surfaces.

\section{PHOTOELECTROCHEMICAL TRANSFORMATIONS see photoelectrocatalysis}

\section{PhOTOELECTROCHEMISTRY}

A term applied to the hybrid field of chemistry that employs techniques, which combine photochemical, and electrochemical methods to examine the redox 
chemistry of the ground and excited states of molecules or ions. In general, it is the chemistry resulting from the interaction of light with electrochemical systems.

See also photoelectrochemical cell, photogalvanic cell, and photovoltaic cell.

\section{Photoelectrode}

An electrode capable of initiating electrochemical transformations after absorbing light quanta.

\section{PhOtOElECTROLYSIS}

See photoelectrocatalysis.

\section{Photoelectrosynthesis}

The process driven by light in the non-spontaneous direction $(\Delta G>0)$ such that radiant energy is stored as chemical energy. This contrasts photocatalysis wherein the process is driven by light in the spontaneous direction $(\Delta G<0)$ such that the light energy is used to overcome the energy of activation of the process.

\section{Photoexcitation}

The act by which an excited state is produced in an entity (molecule or photocatalyst) by the absorption of ultraviolet, visible or infrared radiation.

\section{Photogalvanic Cell}

An electrochemical cell in which current or voltage changes result from photochemically generated changes in the relative concentrations of reactants in a solution phase redox couple.

\section{Photogeneration (of free carriers or excitons)}

The particular case (typical step in solid photocatalysts) of photoexcitation, which leads to formation of free carriers (electron-hole pairs), excitons, electrons in the conduction band or holes in the valence band due to absorption of light in semiconductors or insulators.

\section{Photogenerated CATALYSIS}

A catalytic reaction that involves the production of the catalyst by the absorption of light. The process is catalytic in photons (see Table 1 and Figure 1(a)) contrary to catalyzed photolysis.

The initiation of chemical transformations through the photochemical formation of substances, which (once formed) are catalytically active even without the further action of light.

Note that photogenerated catalysis (see stage $\{15 \mathrm{a}\}$ above) does not require continuous irradiation since the excited state $\mathrm{S}^{+}$is reproduced at the end of each cycle. In this case, the excited state may be created during a pre-irradiation stage and there is no "physical" decay during the course of the reaction.

\section{PHOTOINDUCED CATALYTIC REACTIONS \\ See photogenerated catalysis.}

PHOTOINDUCED CHESORLUMINESCENCE (PhICL effect)

A type of chemiluminescence resulting from the post-adsorption of molecules on a pre-irradiated surface that stimulates luminescence emission in solids. This is not to be confused with the influence of photoadsorption on the photoluminescence of a photocatalyst. As an example, consider the emission of radiation when a (gaseous) substrate, e.g., dihydrogen gas, is chemisorbed on a pre-irradiated semiconductor or insulator such as $\mathrm{ZrO}_{2}$.

\section{PHOTOINDUCED ELECTRON TRANSFER}

An electron transfer from an electronic state produced by the resonant interaction of electromagnetic radiation with matter.

\section{Photoinitiation}

Photoproduction of a free radical or ion capable of initiating a chain reaction such as polymerization.

\section{Photoinitiator}

An agent that initiates, under the action of light, certain chemical transformations and is itself consumed in the reaction or process.

\section{Photolonization}

Ejection of an electron into a surrounding medium induced by the absorption of electromagnetic radiation, from a neutral or positively charged entity (molecular or otherwise).

\section{Photoluminescence}

Luminescence arising from photoexcitation.

\section{PHOTOLYSIS}

A light-induced bond cleavage. This term is often used incorrectly to describe irradiation of a sample, although in the combination flash photolysis this usage is acceptable.

\section{PhOtomineralization}

The process by which an organic substrate is photodegraded into carbon dioxide and water, etc... on absorption of light quanta by the photocatalyst or by the adsorbate.

\section{PHOTON}

The quantum of electromagnetic energy at a given frequency. This energy ( $E=h v)$ is the product of Planck's constant $(h)$ and the frequency of the radiation $(v)$.

\section{PHOTON EMITTANCE}

See photon excitance.

\section{PHOTON EXCITANCE $\left(M_{\mathrm{P}}\right)$}

The photon flow, $\rho$, emitted by an element of the surface containing the source point under consideration divided by the area $(S)$ of that element $(\mathrm{d} \rho / \mathrm{d} S$, simplified expression: $M_{\mathrm{P}}=\rho / S$ when the photon flow is constant over the surface area considered). The SI unit is $\mathrm{s}^{-1} \mathrm{~m}^{-2}$. Alternatively, the term can be used with the amount of photons (mol or its equivalent einstein), the SI unit then being $\mathrm{mol} \mathrm{s}^{-1} \mathrm{~m}^{-2}$. Also called specific photon emission. It was formerly called photon emittance. 
See spectral photon excitance.

See also radiant excitance.

Photon EXPOSURe $\left(H_{\mathrm{p}}\right)$

The photon irradiance, $E_{\mathrm{p}}$, integrated over the time of irradiation ( $\int E_{P} \mathrm{~d} t$, simplified expression: $H_{\mathrm{p}}=E_{\mathrm{p}} t$ when the photon irradiance is constant over the time considered). The SI unit is $\mathrm{m}^{-2}$. Alternatively, the term can be used with the amount of photons (mol or its equivalent einstein), the SI unit then being $\mathrm{mol} \mathrm{m}^{-2}$. For a parallel and perpendicularly incident beam that is neither scattered nor reflected by the target or its surroundings photon fluence $\left(\mathrm{H}_{\mathrm{p}}{ }^{0}\right)$ is an equivalent term.

See also fluence, radiant exposure.

Photon FLOW $(\rho)$

The number of photons (quanta, $N$ ) per unit time. ( $\mathrm{d} N / \mathrm{d} t$, simplified expression: $\rho=N / t$ when the number of photons is constant over the time considered). The SI unit is $\mathrm{s}^{-1}$. Alternatively, the term can be used with the amount of photons (mol or its equivalent einstein), the SI unit being mol s${ }^{-1}$.

See spectral photon flow.

See also radiant power.

\section{Photon Fluence $\left(H_{\mathrm{P}}{ }^{0}\right)$}

The integral of the amount of all photons (quanta), which traverse a small but transparent, imaginary spherical target, divided by the cross-sectional area of this target. The photon fluence rate, $E_{\mathrm{P}}{ }^{0}$, integrated over the duration of the irradiation $\left(\int E_{\mathrm{P}}{ }^{0} \mathrm{~d} t\right.$, simplified expression: $H_{\mathrm{P}}{ }^{0}=E_{\mathrm{P}}{ }^{0} t$ when $E_{\mathrm{P}}{ }^{0}$ is constant over the time considered). Photons per unit area (quanta $\mathrm{m}^{-2}$ ). The SI unit is $\mathrm{m}^{-2}$. Alternatively, the term can be used with the amount of photons (mol or its equivalent einstein), the SI unit then being $\mathrm{mol} \mathrm{m}^{-2}$.

See also fluence.

\section{PhOtON FLUENCE RATE $\left(E_{\mathrm{P}}{ }^{0}\right)$}

The rate of photon fluence. It equals four times the ratio of photon flow, $\rho$, incident on a small transparent, imaginary spherical volume element containing the point under consideration, divided by the surface of that sphere, $S_{\mathrm{K}} \cdot\left(\int_{4 \pi} L_{P} \mathrm{~d} \omega\right.$, simplified expression: $E_{\mathrm{P}}{ }^{0}=4 \rho / S_{\mathrm{K}}$ when the photon flow is a constant over the solid angle considered). The SI unit is $\mathrm{m}^{-2} \mathrm{~s}^{-1}$. Alternatively, the term can be used with the amount of photons (mol or its equivalent einstein), the SI unit then being $\mathrm{mol} \mathrm{m}^{-2} \mathrm{~s}^{-1}$. It reduces to photon irradiance for a parallel and normally incident beam not scattered nor reflected by the target or its surroundings.

See photon irradiance.

See also fluence rate.

\section{PHOTON FLUX}

Same as photon irradiance.

\section{Photonic EFFICIENCY $(\xi)$}

The ratio of the rate of the photoreaction measured for a specified time period (usually the initial condi- tions) to the rate of incident photons at a given wavelength (a protocol for its determination has been given in refs. $[7,20])$. Note that when reporting this quantity the reaction operating conditions must be stated fully. Otherwise, this concept has no photochemical meaning inasmuch as it is difficult to compare the experiments by other research groups when the fraction of light absorbed by the photochemically active component in the system is unknown. This parameter attracts the most attention for any practical application of photocatalysis since it does provide a basis for an engineering efficiency.

See also relative photonic efficiency and photocatalytic efficiency of a system.

\section{PHOTONIC EFFICIENCY SPECTRUM}

A plot of the photonic efficiency of a given photoprocess for a given system measured at the same photon irradiance of incident light versus a measure of the energy of the photons ( $h v$ ) (wavelength, frequency). In practice, the photonic efficiency spectrum is obtained by normalizing the rate of the given process to a certain number of incident photons provided that a linear dependence of the reaction rate on photon irradiance is established by prior experiments.

Note that the photonic efficiency spectrum is not proportional to the spectral absorptance $\mathrm{A}(h v)$ of a system. However, useful information concerning the nature of photoexcitation of a system (in particular of a solid photocatalyst) can be obtained by comparing $\mathrm{A}(h v)$ with the spectral quantum yield for a given system.

\section{PHOTON IRRADIANCE $\left(E_{\mathrm{P}}\right)$}

The photon flow $(\rho)$ incident on an infinitesimal element of surface containing the point under consideration divided by the area $(S)$ of that element $(d \rho / d S$, simplified expression: $E_{\mathrm{P}}=\rho / S$ when the photon flow is constant over the surface considered). The SI unit is $\mathrm{m}^{-2} \mathrm{~s}^{-1}$. Alternatively, the term can be used with the amount of photons ( $\mathrm{mol}$ or its equivalent einstein), the SI unit then being mol m-2 $\mathrm{s}^{-1}$. For a parallel and perpendicularly incident beam that is neither scattered nor reflected by the target or its surroundings photon fluence rate $\left(E_{\mathrm{p}}{ }^{0}\right)$ is an equivalent term.

See spectral photon irradiance.

See also irradiance.

\section{Photon RADIANCE $\left(L_{\mathrm{P}}\right)$}

For a parallel beam it is the photon flow $(\rho)$ leaving or passing through an infinitesimal transparent element of surface in a given direction from the source divided by the orthogonally projected area of the element in a plane normal to the given direction of the beam, $\theta$, $\left((d \rho / d S) / \cos \theta\right.$, simplified expression: $L_{\mathrm{P}}=\rho /(S \cos \theta)$ when the photon flow is constant over the surface area considered). The SI unit is $\mathrm{m}^{-2} \mathrm{~s}^{-1}$. 
For a divergent beam propagating in an elementary cone of the solid angle $\mathrm{d} \omega$ containing the direction $\theta$, the photon radiance is $\mathrm{d}^{2} \rho /(\mathrm{d} \omega \mathrm{d} S \cos \theta)$, with the SI unit $\mathrm{m}^{-2} \mathrm{~s}^{-1} \mathrm{sr}^{-1}$. Alternatively the term can be used with the amount of photons (mol or its equivalent einstein), the SI unit then being $\mathrm{mol} \mathrm{m}^{-2} \mathrm{~s}^{-1}$ and $\mathrm{mol} \mathrm{m}^{-2}$ $\mathrm{s}^{-1} \mathrm{sr}^{-1}$, respectively.

See spectral photon radiance.

See also radiance.

\section{Photooxidation}

Oxidation reactions induced by light. Common processes are:

(1) The loss of one or more electrons from a chemical species as a result of photoexcitation of that species.

(2) The reaction of a substance with a reactive oxygen species (ROS: e.g., • $\mathrm{OH}, \mathrm{O}_{2}{ }^{-} \bullet, \mathrm{HOO} \bullet, \mathrm{H}_{2} \mathrm{O}_{2}$, ${ }^{1} \mathrm{O}_{2}$ ) under the influence of light. When oxygen remains in the product this latter process is also called photooxygenation.

(3) Reactions in which neither the substrate nor the molecular oxygen are referred to as photoinitiated oxidations.

Compare with photoreduction.

\section{Photooxygenation}

Incorporation of molecular oxygen into a molecular entity. There are three common mechanisms:

Type I: the reaction of triplet molecular oxygen $\left({ }^{3} \mathrm{O}_{2}\right)$ with radicals formed photochemically.

Type II: the reaction of photochemically produced singlet molecular oxygen $\left({ }^{1} \mathrm{O}_{2}\right)$ with molecular entities to give rise to oxygen containing molecular entities.

Type III: the third mechanism proceeds by electron transfer to produce the superoxide radical anion $\left(\mathrm{O}_{2}{ }^{-} \bullet\right)$ as the reactive species.

Compare with photooxidation.

\section{PHOTOPHYSICAL PROCESS}

Photoexcitation and subsequent events, which lead from one electronic state to another electronic state of a molecular entity (or solid) through radiative and nonradiative transitions. No chemical change results.

\section{Photoradiocatalysis}

Radiocatalysis taking place under the effect of the simultaneous action of quanta of light and ionizing radiation. There is also the case when the ionizing radiation alone causes the emission of light that is chemically active (e.g., as may occur in the Cherenkov light emission).

\section{PHOTOREACTION \\ See photochemical reaction.}

\section{PHOTOREDUCTION}

Reduction reactions induced by light. Common processes are:
(1) Addition of one or more electrons to a photoexcited species.

(2) The photochemical hydrogenation of a substance.

Reactions in which the substrate is not electronically excited are sometimes called photoinitiated reductions.

\section{Photosensitization}

The process by which a photochemical change or photophysical process occurs in one (molecular) entity as a result of initial absorption of radiation by another (molecular) entity called a photosensitizer. In mechanistic photochemistry, the term is limited to cases in which the photosensitizer is not consumed in the reaction.

See energy transfer.

\section{PHOTOSENSITIZED REACTION}

A reaction initiated by photosensitization.

See catalyzed photolysis.

\section{Photosensitizer (sensitizer)}

A substrate that absorbs radiation and subsequently initiates a photochemical or photophysical process in another system, with the substrate being fully recovered. In case where a chemical alteration has occurred, the photosensitizer is equivalent to a photocatalyst.

See photosensitization.

\section{PhOtostationary STATE}

A steady state reached by a reacting chemical system when light has been absorbed by at least one of the components. At the steady state, the rates of formation and disappearance are equal for each of the transient (molecular) entities formed.

Photosynthesis (in relation to abiogenic systems and objects)

Typically the synthesis of more complex compounds from less complex ones by the action of light.

Note that in some utilization of this term, photosynthesis is not necessarily associated with photocatalytic transformations, but does include photochemical transformations as a possible component.

\section{PHOTOTHERMAL EFFECT}

An effect produced by photoexcitation of an entity resulting partially or totally in the production of heat.

\section{PhOtOTheRMOCATAlysis}

Catalysis occurring by the simultaneous or sequential action of light and thermal energy. Also a catalytic phenomenon that occurs in circumstances when the system is also heated by light. In case of light with a high photon flux, it is possible that not only photochemical (quantum) but also thermochemical processes proceed as a result of the system being heated through absorption of light quanta. This often occurs upon exposure 
of a heterogeneous (photo)catalyst to pulsed IR-light or pulsed laser emission.

\section{PHOTOTHERMOgRAPHY}

A process utilizing both light and heat, simultaneously or sequentially, for image recording.

\section{Photovoltaic CELL}

A solid-state device, usually a semiconductor (e.g., silicon), that absorbs photons of energies greater than or equal to the bandgap energy and simultaneously produces electric power.

Compare photogalvanic cell.

\section{Physical pathway}

In photocatalysis, the physical pathway is the set of physical processes, which lead to the relaxation of the electronically photoexcited system without chemical transformations. In particular, such processes are radiative and non-radiative relaxation in molecules and surface complexes from an excited state to the ground state, from recombination and trapping of free charge carriers, from the recombination decay of trapped carriers (particularly, recombination decay of carriers trapped by surface active centers), etc.

See chemical pathway.

\section{PHYSISORPTION}

Adsorption of an entity (molecule, atom or ion) onto a surface by way of a physical interaction-usually van der Waals forces, dipolar interactions, etc...-between a surface and the entity. An example is the physisorption of a hydrocarbon onto a metal-oxide particle surface.

\section{Piezoluminescence}

Luminescence observed when certain solids are subjected to a change in pressure.

See triboluminescence.

\section{POINT OF ZERO CHARGE (PZC)}

$\mathrm{pH}$ at which the charge on a semiconductor (or insulator) surface is zero; that is, the surface is electrically neutral. Note that the PZC can be affected by surface adsorbed species.

\section{PRIMARY PHOTOCHEMICAL PROCESS (primary photore- action)}

Any elementary chemical process undergone by an electronically excited (molecular) entity and yielding a primary photoproduct.

See primary (photo)process.

\section{PRIMARY (PHOTO)PROCESS}

The term primary (photo)process for a photophysical process is apt to lead to inconsistencies, and its use is therefore discouraged.

See primary photochemical process.

\section{PRIMARY (PHOTO)PRODUCT}

The first observable chemical entity which is produced in the primary photochemical process and which is chemically different from the reactant.

See primary (photo)process.

QUANTUM (of radiation)

An elementary particle of electromagnetic energy in the sense of wave-particle duality.

See photon.

\section{QUANTUM EFFICIENCY $(\eta)$}

For a primary photochemical process, quantum efficiency is identical to quantum yield in homogeneous photochemistry.

In heterogeneous photocatalysis, it refers to the $p h o$ tonic efficiency when the relevant systems are irradiated with broadband radiation.

See efficiency.

QUANTUM YIELD (in photochemistry; $\Phi$ )

The number of defined events, which occur per photon absorbed by the system at a specified wavelength. The integral quantum yield is:

$$
\Phi=\frac{\text { Number of events }}{\text { Number of photons absorbed }}
$$

For a photochemical reaction,

$\Phi=\frac{\text { Amount of reactant consumed or product formed }}{\text { Amount of photons absorbed }}$

The differential quantum yield is (in terms of rates),

$$
\Phi=\frac{\mathrm{d}[x] / \mathrm{d} t}{n}
$$

where $\mathrm{d}[x] / \mathrm{d} t$ is the rate of change of a measurable quantity, and $n$ is the amount of photons (mol or its equivalent einstein) absorbed per unit time. $\Phi$ can be used for photophysical processes or photochemical reactions.

See also efficiency.

QUANTUM YIELD ( $\Phi$, of a photocatalytic process)

Defined exactly as the quantum yield of a photochemical process; that is, the number of molecules of a given product formed (or alternatively of a given reactant disappeared) per photon of light of a given wavelength that is absorbed by the photocatalyst:

$$
\Phi=\frac{N_{\text {molec }}}{n_{\text {ph }}}
$$

where $N_{\text {molec }}$ is a quantity of reacted molecules or product molecules formed, and $n_{\mathrm{ph}}$ is the quantity of absorbed photons.

This definition can be used for both homogenous and heterogeneous photocatalysis. It must be noted that for consistency both quantities must be evaluated 
under identical conditions, and preferably at the same time. In practice, the terms defining the quantum yield are typically "rates". The numerator represents the reaction rate and the denominator represents the rate of the photon flow absorbed:

$$
\Phi=\frac{R^{V}}{R^{V} \mathrm{ph}}
$$

where $R^{V}$ is the volumetric rate corresponding to a given reactant (or product), and $R^{V}$ ph is the volumetric rate of photons absorbed by the reactant or by the catalyst.

For photochemical processes in homogeneous systems the reaction rate and the photon absorption rate are phenomena occurring in the volume of the system and therefore the respective rates can be determined with reference to the system volume.

For photocatalytic processes, which occur in macro heterogeneous systems, a method for estimating quantum yields in photocatalytic systems has been described and discussed in [9]. Other descriptions have also been reported $[39,50]$. In suspensions or in homogeneous solutions it is possible to determine the rate of the reaction (for example, by use of an integrating sphere method $[50,51])$ as well as the fraction of light absorbed in a given volume of suspension and hence determine the rate of photon absorption.

For powdered heterogeneous systems, the rate of photon absorption can also be measured using a blackbody-like photocatalytic reactor simultaneously with the rate of the photocatalytic reaction itself. Thus, also in such cases it is possible to obtain the quantum yield of a given process.

Note that in a system where both a photocatalytic reaction and a parallel direct photolysis (due to absorption of light by the substrate, e.g., in solution) take place, two different quantum yields must be determined, one for the heterogeneous (photocatalytic) reaction and the other for the homogeneous photolysis. A method for separating the absorption by the solid catalyst from the absorption by the fluid reactants containing phase has been described in [52].

Note also that in experiments to measure the quantum yield of a surface photochemical process in a heterogeneous system, the actual illuminated surface, $S$, of the particle dispersion is always an unknown quantity. Clearly the theoretical estimate of $S$ depends on the dispersion of the sample and the extent to which it absorbs and reflects light. Moreover, illumination of the sample's surface is strongly non-uniform, whereas the definition of the quantum yield in heterogeneous systems requires that the surface be uniformly irradiated. It is relevant to note, however, that the quantum yield $\Phi$ does not depend on $S$ as long as the rate of reaction is directly proportional to the irradiance of the incident light [17]. Indeed, the rate of the photochemi- cal process on a unit of the uniformly irradiated surface can be represented as:

$$
d(\text { rate })=\phi_{\lambda} \rho(S) \mathrm{d} S
$$

The total reaction rate is then given by:

$$
\text { Rate }=\int_{S} \phi_{\lambda} \rho(S) \mathrm{d} S
$$

where $\Phi_{\lambda}$ is the "true" quantum yield at wavelength $\lambda$ on the uniformly irradiated elementary surface unit independent of photon flow, $\rho(S)$ is the light irradiance impinging on the given surface unit, and $d S$ is the elementary surface unit. The quantum yield can then be determined as:

$$
\Phi=\frac{\int_{S} \phi_{\lambda} \rho(S) \mathrm{d} S}{\int_{S} \rho(S) \mathrm{d} S}
$$

Now, if the rate is directly proportional to the photon flow, $\phi_{\lambda}$ is constant and thus

$$
\Phi=\frac{\phi_{\lambda} \int_{S} \rho(S) \mathrm{d} S}{\int_{S} \rho(S) \mathrm{d} S}=\phi_{\lambda}
$$

That is, the quantum yield does not depend on the irradiated surface (otherwise, it would be) and is equal to the actual quantum yield of the photochemical reaction on the elementary surface unit. Also, the latter condition is essential in order to avoid a dependence of the quantum yield of the surface photochemical reaction on light irradiance (or photon flow). The dependence of the rate of reaction $(\mathcal{R})$ occurring on the elementary surface unit on photon flow $\rho$ can generally be represented by:

$$
\mathcal{R}=\text { (const) } \rho^{m}
$$

In this case, the expression for the quantum yield is given by

$$
\Phi=(\text { const })^{\prime} \rho^{m-1}
$$

Clearly, for $m<1$ the quantum yield of a surface process decreases as the photon flow increases. If the order of the reaction with respect to photon flow $m$ is unity, the quantum yield becomes independent of photon flow and yields the maximal value of $\Phi$.

The typical experimental dependence of the elementary reaction rate on the concentration of reagent can also be represented by the Langmuir type saturation curve:

$$
\mathcal{R}=\frac{k \mathrm{~K} C}{1+\mathrm{K} C}
$$

It is recommended that the quantum yield of a surface photochemical reaction be measured in the region of saturation of the rate dependence on reagent concentration. That is, at $\mathrm{KC} \gg 1$ so as to avoid the 
dependence of the quantum yield on reagent concentration, and to permit the reported data to be compared with those obtained by other research groups. Moreover, for all heterogeneous systems so far tested, it was established that because of the interdependence of reaction rate on photon flow and on reagent concentration, the condition $\mathrm{KC} \gg 1$ leads to the independence of the quantum yield on photon flow. In this manner, $\Phi$ can then be used to compare results with those obtained in other laboratories [9, 23].

\section{QUENCHER}

An entity (molecular or otherwise) that deactivates (quenches) an excited state of another entity, either by energy transfer or electron transfer, or by a chemical mechanism.

See quenching.

\section{QUENCHING}

The deactivation of an excited (molecular) entity intermolecularly by an external environmental influence (such as a quencher) or intramolecularly by a substituent through a nonradiative process. When the external environmental influence (quencher) interferes with the behavior of the excited state after its formation, the process is referred to as dynamic quenching. Common mechanisms include energy transfer and electron transfer, etc.... When the environmental influence inhibits the excited state formation the process is referred to as static quenching.

\section{RADIANCE $(L)$}

For a parallel beam it is the radiant power, $P$, of all wavelengths leaving or passing through an infinitesimal element of surface in a given direction from the source divided by the orthogonally projected area of the element in a plane normal to the given direction of the beam, $\theta,((\mathrm{d} P / \mathrm{d} S) / \cos \theta$, simplified expression: $L=P /(S \cos \theta)$ when the radiant power is constant over the surface area considered). The SI unit is $\mathrm{W} \mathrm{m}^{-2}$. Note that $L=\int L_{\lambda} \mathrm{d} \lambda$, where $L_{\lambda}$ is the spectral radiance at wavelength $\lambda$. For a divergent beam propagating in an elementary cone of the solid angle $\mathrm{d} \omega$ containing the given direction $(\theta)$ the radiance is $d^{2} P /(\mathrm{d} \omega \mathrm{d} S \cos \theta)$, with SI units of $\mathrm{W} \mathrm{m} \mathrm{m}^{-2} \mathrm{sr}^{-1}$.

See also photon flow, photon radiance, and spectral radiance.

\section{RADIANT EMITTANCE}

See radiant excitance.

\section{RADIANT ENERGY $(Q)$}

The total energy emitted, transferred or received as radiation of all wavelengths in a defined period of time $\left(Q=\int Q_{\lambda} \mathrm{d} \lambda\right)$. It is the product of radiant power, $P$, and time, $t$; namely $Q=P t$ when the radiant power is constant over the time considered. The SI unit is J.

See also spectral radiant power.

\section{RADIANT (ENERGY) FLUX}

Although flux is generally used in the sense of the "rate of transfer of fluid, particles or energy across a given surface", the radiant energy flux has been adopted by the IUPAC as equivalent to radiant power, $P,(P=\mathrm{d} Q / \mathrm{d} t$, simplified expression: $P=Q / t$ when the radiant energy, $Q$, is constant over the time considered), units: $\mathrm{J} \mathrm{s}^{-1}$.

See also photon flow, photon radiance, radiant energy, and spectral radiant flux.

\section{RADIANT EXCITANCE $(M)$}

The radiant power $(P)$ emitted at all wavelengths by an element of the surface containing the source point under consideration divided by the surface area $(S)$, of that element. $(\mathrm{d} P / \mathrm{d} S$, simplified expression: $M=P / S$ when the radiant power is constant over the surface area considered.) It is the integration of the radiant power leaving a source of the solid angle and over the whole wavelength range. The SI unit is $\mathrm{W} \mathrm{m}^{-2}$. Note that $M=\int M_{\lambda} \mathrm{d} \lambda$, where $M_{\lambda}$ is the spectral radiant excitance at wavelength $\lambda$. Formerly called radiant emittance. Same as spherical radiant excitance.

See also photon excitance, spectral radiant excitance.

\section{RADIANT EXPOSURE $(H)$}

The irradiance, $E$, integrated over the time of irradiation ( $\int E \mathrm{~d} t$, simplified expression: $H=E t$ when the irradiance is constant over the time considered) [units: $\left.\mathrm{J} \mathrm{m}^{-2}\right]$. For a parallel and perpendicular incident beam that is neither scattered nor reflected by the target or its surroundings, fluence $\left(H_{0}\right)$ is an equivalent term.

\section{RADIANT INTENSITY $(I)$}

Radiant (energy) flux or radiant power $(P)$ at all wavelengths per unit solid angle, $\omega$. The radiant power emitted in a given direction by a source or an element of the source in an infinitesimal cone containing the given direction divided by the solid angle of the cone ( $\mathrm{d} P / \mathrm{d} \omega$, simplified expression: $I=P / S$ when the radiant power is constant over the surface area considered). The SI unit is $\mathrm{W} \mathrm{sr}{ }^{-1}$. Note that $I=\int I_{\lambda} \mathrm{d} \lambda$, where $I_{\lambda}$ is the spectral radiant intensity at wavelength $\lambda$.

See also spectral radiant intensity.

\section{RADIANT POWER $(P)$}

Same as radiant (energy) flux. That is, power emitted, transferred or received as radiation. The SI unit is $\mathrm{J} \mathrm{s}^{-1}=\mathrm{W}$.

See spectral radiant power.

\section{RADIANT DOSE}

The radiant energy (in Joules) or quantity of photons (in mols) absorbed per unit area or unit volume by an object irradiated during a given exposure time.

\section{RADIATION EFFICIENCY}

The ratio of the number of product molecules formed of a given radiocatalytic process (alternatively, 
the number of molecules of a given reactant disappearing) for every $100 \mathrm{eV}$ of energy of the high-energy radiation incident on the reacting system.

\section{RADIANTIONLESS (NONRADIATIVE) DEACTIVATION (Decay)}

Loss of electronic excitation energy without photon emission or chemical change.

\section{RADIATIONLESS (NONRADIATIVE) TRANSITION}

A transition between two states of a system without photon emission or absorption.

Compare with radiative transition.

\section{RADIATION YIELD OF A RADIOCATALYTIC PROCESS}

The number of product molecules formed of a given radiocatalytic process (alternatively, the number of molecules of a given reactant disappearing) for every $100 \mathrm{eV}$ of energy of the high-energy radiation absorbed by the radiocatalyst.

\section{RADIATIVE ENERGY TRANSFER}

Transfer of excitation energy by radiative deactivation of a donor (molecular) entity and reabsorption of the emitted light by an acceptor (molecular) entity. The probability of transfer is given approximately by

$$
P_{\mathrm{rt}}=[A] \chi J
$$

where $J$ is the spectral overlap integral, $[A]$ is the concentration of the acceptor, and $\chi$ is the specimen thickness. This type of energytransfer depends on the shape and size of the vessel utilized.

See energy transfer.

\section{RADIATIVE TRANSITION}

A transition between two states of an (molecular) entity, the energy difference being emitted or absorbed as a photon.

See luminescence.

Compare with radiationless deactivation and radiationless transition.

\section{RADIOACTIVATION OF CATALYSTS}

The alteration of the catalytic activity (or selectivity) of a substance after its exposure to ionizing radiation.

\section{RADIOADSORPTION}

Adsorption of a substance at solid or liquid interfaces induced by absorption of high-energy radiation by the solid or liquid adsorbent.

\section{RADIOCATALYSIS}

Catalysis occurring under the action of quanta of ionizing radiation absorbed by the radiocatalytic substance (that is, the radiocatalyst).

\section{RADIOCATALYST}

See radiocatalysis.

\section{RADIODESORPTION}

Desorption of a substrate from solid or liquid interfaces induced by absorption of high-energy radiation by the solid or liquid adsorbent.

\section{RADIOGENERATED CATALYSIS}

A catalytic reaction that involves the production of the catalyst by the absorption of ionizing radiation. The process is catalytic in the quanta of the ionizing radiation.

The initiation of chemical transformations through the radiochemical formation of substances, which (once formed) are catalytically active even without the action of the ionizing radiation.

\section{RADIOINDUCED (INITIATED, STIMULATED) ADSORP- TION}

see radioadsorption.

\section{RADIOINDUCED (INITIATED, STIMULATED) DESORP-} TION

see radiodesorption.

\section{RADIOLUMINESCENCE}

Luminescence arising from excitation by highenergy particles or radiation.

\section{RADIOLYSIS}

Bond cleavage induced by high-energy radiation. More loosely the term is also used for any chemical process brought about by high-energy radiation. The term has also been used to refer to the irradiation technique itself (e.g., pulse radiolysis).

\section{RADIOMODIFICATION OF CATALYSTS} see radioactivation of catalysts.

\section{RADIOSENSITIZATION}

The process by which a chemical change or physical process occurs in one (molecular) entity as a result of initial absorption of high-energy radiation by another (molecular) entity called the radiosensitizer

\section{RADIOSENSITIZER}

See radiosensitization

\section{RADIOSTIMULATED DIFFUSION}

Increase of diffusion of an adsorbate under the action of quanta of ionizing irradiation absorbed by either the adsorbent and/or the adsorbate.

\section{RADIOTHERMAL CATALYSIS}

Radiocatalysis under the action of large radiation fluxes that simultaneously heat the system as a consequence of the absorption of radiation energy.

\section{RATE OF SURFACE RECOMBINATION $(s)$}

As in solid-state physics, it is related to the decay of carriers at the crystal surface and corresponds to the inverse of the lifetime of free carriers in the bulk of the crystalline particle. The greater $\mathrm{s}$ is the smaller is the stationary concentration of photocarriers at the 
surface (and vice versa). In some theoretical models, the reaction rate of photocatalyzed processes is considered as the specific component $s$ (SI unit is: $\mathrm{m} \mathrm{s}^{-1}$ but sometimes $\mathrm{cm} \mathrm{s}^{-1}$ is used).

See surface recombination, and surface concentration carriers.

\section{RATE OF (PHOTO)GENERATION (of carriers; g)}

The number of carriers (or electron-hole pairs, or excitons) produced per unit time per unit volume of a solid photocatalyst particle due to the absorption of actinic light. In the general case, the rate of photogeneration in the bulk of optical uniform photocatalyst particle depends on the distance $(x)$ from the irradiated surface.

$$
g(x)=\alpha E_{p}(1-R) \exp [-\alpha x]
$$

where $\alpha$ is the absorption coefficient, $E_{\mathrm{p}}$ is the photon irradiance, and $R$ is the reflectance. This rate $\mathrm{g}(x)$ is related to the concentration of photo-electrons $\left(n_{\mathrm{e}}\right)$ and photo-holes $\left(n_{\mathrm{h}}\right)$ as,

$$
\begin{aligned}
& n_{\mathrm{e}}=\mathrm{g}(x) \tau_{\varepsilon} \\
& n_{\mathrm{h}}=\mathrm{g}(x) \tau_{\mathrm{h}}
\end{aligned}
$$

where the $\tau_{\mathrm{e}}$ and $\tau_{\mathrm{h}}$ are the lifetimes of the relevant free photocarriers. In kinetic studies of photocatalyzed reactions, the rate of generation is typically considered as the constant $\mathrm{g}=\alpha E_{\mathrm{p}}$ (uniform generation), which corresponds to the so-called weak light absorption case $\left(\alpha_{x} \ll 1\right)$. [units of $g$ are mol m${ }^{-3} \mathrm{~s}^{-1}$ or $\mathrm{m}^{-3} \mathrm{~s}^{-1}$.]

\section{REACTIVE OXYGEN SPECIES (ROS)}

Species formed by the absorption of light quanta by a semiconductor or insulator followed by oxidation of the surface hydroxyl groups or water by the valence band holes and/or by the reduction of surface chemisorbed molecular oxygen by the conduction band electrons (e.g., $\bullet \mathrm{OH}, \mathrm{HOO} \bullet, \mathrm{O}_{2}^{-\bullet},{ }^{1} \mathrm{O}_{2}$ ).

RECOMBINATION (of free electrons and holes in solid photocatalyst)

The disappearances of free electrons and free holes due to the transition of electrons from the conduction band to the valence band. The recombination rate of photocarriers (that is, the number of recombination events in unit time in unit volume) determines the stationary concentration of non-equilibrium carriers in an irradiated solid photocatalyst, and thus determines the rates and quantum yields of a majority of heterogeneous photocatalytic reactions.

The recombination of carriers obeys the energy and momentum conservation laws. Consequently, in wide bandgap solids the radiative recombination is more favorable. In real solids that contain lattice imperfections (lattice defects) at moderate intensity of photoexcitation, recombination through such defects (recombina- tion centers) is more efficient than direct band-to-band recombination.

\section{RECOMbINATION OF PHOTOCARRIERS (via defects)}

Typically, the two-step transition of an electron from the conduction band to a defect center, and then further to the valence band, or from the defect center to the valence band (first step) and a subsequent transition of the conduction band electron to the defect center (second step).

See also: recombination center.

\section{RECOMBINATION CENTER}

A defect in semiconductors and insulators that is capable of sequential capture of free carriers of both sign with high efficiency. It provides a path for the recombination of free electrons and holes at high rates. Typically, in imperfect wide-bandgap solids the rate of photoinduced carrier recombination through recombination centers at moderate photon irradiance is much greater than the rate of band-to-band recombination.

\section{RED SHIFT \\ Informal term for bathochromic shift.}

\section{REFLECTANCE $(R)$}

The fraction of light reflected by a system; $R$ is the term in the sum: $\mathrm{A}+T+R=1$, where $\mathrm{A}$ is the absorptance and $T$ is the transmittance.

\section{REGULAR SURFACE}

A perfect surface of a solid without defects of any sort.

Note that rigorously speaking, a regular surface is a theoretical notion. In practice, however, one can use this term to refer to local space regions of real relaxed and reconstructed surfaces if the perturbations caused by the nearest adjacent (surface) defects can be neglected under some given experimental conditions or in a given theoretical model.

See also: surface.

\section{REGULAR SURFACE SITE}

Any zero-dimensionless site (atom, ion or interstitial position) at a regular surface.

Note that a regular surface site can be a photocat alytic center (site) in the ground (not active) state in some particular cases of heterogeneous photocatalysis as, for example, in the case when pre-physisorbed molecules trap photocarriers in the course of the photocatalyzed reactions.

\section{RELATIVE PHOTONIC EFFICIENCY $\left(\xi_{\text {rel }}\right)$}

The ratio of the photonic efficiency $(\xi)$ of a chemical process under study to the corresponding photonic efficiency of a standard process under otherwise identical conditions (for the protocol for its determination 
see refs. $[8,9])$. When the processes are carried out at constant photon flow, the relative photonic efficiency can also be estimated as the ratio of the corresponding initial rates of reaction.

\section{RELAXATION}

Passage of an excited or otherwise perturbed system towards or into thermal equilibrium with its environment.

See radiationless deactivation, radiationless transition, and radiative transition.

\section{SACRIFICIAL ACCEPTOR}

An (molecular) entity that acts as the electron acceptor in a photoinduced electron transfer process and is not restored in a subsequent oxidation process, but is destroyed by irreversible chemical conversion.

\section{SACRIFICIAL DONOR}

An (molecular) entity that acts as the electron donor in a photoinduced electron transfer process and is not restored in a subsequent reduction process, but is destroyed by irreversible chemical conversion.

\section{SEMICONDUCTOR}

Phenomenologically, a semiconductor is a material whose electrical conductivity increases with increasing temperature owing to the thermal generation of free charge carriers. The bandgap of an intrinsic semiconductor can be as high as about $2 \mathrm{eV}$.

An intrinsic semiconductor is a material with negligible concentration of defects and impurities, and for which thermal excitation leads to band-to-band generation of both electrons and holes with identical concentrations of both types of carriers. This requires a small bandgap energy of the semiconductor. For example, the intrinsic Si semiconductor whose bandgap energy is $1.2 \mathrm{eV}$ demonstrates significant conductivity at $500 \mathrm{C}$ only.

An $n$-type semiconductor is a material in which electrons are the majority carriers owing to the presence of shallow donor intrinsic defects and/or impurities in the lattice.

A p-type semiconductor is a material in which the holes are the majority carriers owing to the presence of shallow acceptor intrinsic defects and/or impurities in the lattice.

Note that the presence of defects and impurities in $\mathrm{n}$ - or $\mathrm{p}$ - type semiconductors leads to the observation of semiconductor behavior in materials with a wider bandgap than in intrinsic semiconductors. For example, $\mathrm{ZnO}$ and $\mathrm{TiO}_{2}$, which have a bandgap of about $3 \mathrm{eV}$, behave as $n$-type semiconductors.

\section{SENSITIZED PHOTOREACTIONS}

See catalyzed photolysis, and photocatalysis.

\section{SENSITIZER}

see photosensitizer.

\section{SENSITIZATION}

See photosensitization.

\section{SHALLOW (ENERGY) TRAP}

An energy level (of trapped carrier) within the bandgap near the bottom of the conduction band (electron shallow trap) and near the top of the valence band (hole shallow trap) at an energy such that the $E_{\text {trap }} \sim k T$, that is close to the energy of thermal excitation of a solid at a given temperature. The dominant pathway of shallow trap decay is thermal ionization yielding a free carrier and an empty trap. The photocatalytic (or photoadsorption) active center of a given type can, in principle, be described as a shallow trap in terms of solid-state physics.

\section{SINGLET MOLECULAR OXYGEN}

The oxygen molecule (dioxygen), $\mathrm{O}_{2}$, in an excited singlet state. The ground state of $\mathrm{O}_{2}$ is a triplet ${ }^{3} \Sigma_{\mathrm{g}}{ }^{-}$. The two metastable singlet states derived from the ground state configuration are ${ }^{1} \Delta_{\mathrm{g}}$ and ${ }^{1} \Sigma_{\mathrm{g}}{ }^{-}$.

The term singlet oxygen alone, without mention of the chemical species, is discouraged since it can also refer to an oxygen atom in $\mathrm{a}^{1} \mathrm{~S}$ or ${ }^{1} \mathrm{D}$ excited state. While the oxygen atom ground state is a triplet ${ }^{3} \mathrm{P}$ state, the ${ }^{1} \mathrm{~S}$ or ${ }^{1} \mathrm{D}$ states are also derived from the ground state configuration.

\section{SINGLET STATE}

An electronic state whose total electron spin quantum number is zero.

\section{SINGLET-TRIPLET ENERGY TRANSFER}

Transfer of excitation energy from an electronically excited donor in a singlet state to produce an electronically excited acceptor in a triplet state.

See energy transfer.

\section{SOLAR ENERGY CONVERSION}

The ratio of the Gibbs energy for a process per unit time per $\mathrm{m}^{2}$ of surface exposed to the sun to the solar irradiance, $E$, integrated between $\lambda=0$ and $\lambda=\infty$.

\section{SPACE CHARgE REgION}

Region of excess charge in a semiconductor resulting from electron flow from the semiconductor to the solution phase when the Fermi energy level of the semiconductor lies above that of the solution phase. The resulting electric field that forms in the space charge region is represented as band bending.

\section{SPECTRAL IRRADIANCE $\left(E_{\lambda}\right)$}

Irradiance, $E$, at wavelength $\lambda$ per unit wavelength interval. The SI unit is $\mathrm{W} \mathrm{m}^{-3}$, but a commonly used unit is $\mathrm{W} \mathrm{m}^{-2} \mathrm{~nm}^{-1}$. 


\section{SPECTRAL PHOTON EFFECTIVENESS}

The reciprocal of the photon fluence rate, $E_{\mathrm{p}}{ }^{0}$, at wavelength $\lambda$ causing an identical photoresponse $\Delta y$ per unit time $(\Delta y / \Delta t)$. The effectiveness spectrum is directly proportional to the conversion spectrum of the sensory pigment, if spectral attenuance is negligible.

\section{SPECTRAL PHOTON EXCITANCE $\left(M_{\mathrm{p} \lambda}\right)$}

The photon excitance, $M_{\mathrm{p}}$, at wavelength $\lambda$ per unit wavelength interval. The SI unit is $\mathrm{s}^{-1} \mathrm{~m}^{-3}$, but a commonly used unit is $\mathrm{s}^{-1} \mathrm{~m}^{-2} \mathrm{~nm}^{-1}$. Alternatively, the term can be used with the amount of photons (mol or its equivalent einstein), the SI unit then being mol s${ }^{-1}$ $\mathrm{m}^{-3}$ and the common unit being $\mathrm{mol} \mathrm{s}^{-1} \mathrm{~m}^{-2} \mathrm{~nm}^{-1}$.

\section{SPECTRAL PHOTON FLOW $\left(\rho_{\lambda}\right)$}

The photon flow, $\rho$, at wavelength $\lambda$ per unit of wavelength interval. The SI unit is $\mathrm{s}^{-1} \mathrm{~m}^{-1}$, but a commonly used unit is $\mathrm{s}^{-1} \mathrm{~nm}^{-1}$. Alternatively, the term can be used with the amount of photons (mol or its equivalent einstein), the SI unit then being mol s $\mathrm{s}^{-1} \mathrm{~m}^{-1}$ and the common unit being mol s $\mathrm{sm}^{-1}$.

\section{SPECTRAL PHOTON FLUX (photon irradiance) $\left(E_{\mathrm{p} \lambda}\right)$}

The photon irradiance, $E_{\mathrm{p}}$, at wavelength $\lambda$ per unit wavelength interval. The SI unit is $\mathrm{m}^{-3} \mathrm{~s}^{-1}$, but a commonly used unit is $\mathrm{m}^{-2} \mathrm{~nm}^{-1} \mathrm{~s}^{-1}$. Alternatively, the term can be used with the amount of photons (mol or its equivalent einstein), the SI unit then being $\mathrm{mol} \mathrm{m}^{-3}$ $\mathrm{s}^{-1}$ and the common unit being mol m $\mathrm{m}^{-2} \mathrm{~nm}^{-1} \mathrm{~s}^{-1}$.

\section{SPECTRAL (DEPENDENCE OF) PHOTONIC EFFICIENCY}

The dependence of the photonic efficiency on the photon energy (wavelength or frequency) of the actinic light for a given photoreaction obtained at a certain photon irradiance of the incident light (photon flux) in a given spectral range of photoexcitation.

Note (i) that in practice, it is normalized to a certain photon flux provided that the reaction rate scales linearly with the photon irradiance; (ii) in reality, some interesting information concerning the photoexcitation of a solid photocatalyst can be obtained from a comparison of the spectral quantum yield with the spectral photonic efficiency.

\section{SPECTRAL (DEPENDENCE OF) QUANTUM YIELD}

The dependence of the quantum yield on the photon energy (wavelength or frequency) of the actinic light for a given photoreaction. To obtain information on the spectral variation of activity of a photocatalyst, the reaction rate measured to calculate the quantum yield must scale linmearly with photon flow at all wavelengths.

\section{SPECTRAL PHOTON RADIANCE $\left(L_{\mathrm{p} \lambda}\right)$}

The photon radiance, $L_{\mathrm{p}}$, at wavelength $\lambda$ per unit wavelength interval. The SI unit is $\mathrm{s}^{-1} \mathrm{~m}^{-3} \mathrm{sr}^{-1}$, but a commonly used unit is $\mathrm{s}^{-1} \mathrm{~m}^{-2} \mathrm{sr}^{-1} \mathrm{~nm}^{-1}$. Alternatively, the term can be used with the amount of photons (mol or its equivalent einstein), the SI unit then being mol s$~^{-1} \mathrm{~m}^{-3} \mathrm{sr}^{-1}$ and the common unit being $\mathrm{mol} \mathrm{s}^{-1}$ $\mathrm{m}^{-2} \mathrm{sr}^{-1} \mathrm{~nm}^{-1}$.

\section{SPECTRAL RADIANCE $\left(L_{\lambda}\right)$}

The radiance, $L$, at wavelength $\lambda$ per unit wavelength interval. The SI unit is $\mathrm{W} \mathrm{m}^{-3} \mathrm{sr}^{-1}$, but a commonly used unit is $\mathrm{W} \mathrm{m}^{-2} \mathrm{sr}^{-1} \mathrm{~nm}^{-1}$.

\section{SPECTRAL RADIANT EXCITANCE $\left(M_{\lambda}\right)$}

The radiant excitance, $M$, at wavelength $\lambda$ per unit wavelength interval. The SI unit is $\mathrm{W} \mathrm{m}^{-3}$, but a commonly used unit is $\mathrm{W} \mathrm{m}^{-2} \mathrm{~nm}^{-1}$.

\section{SPECTRAL RADIANT FLUX}

Same as spectral radiant power.

\section{SPECTRAL RADIANT INTENSITY $\left(I_{\lambda}\right)$}

The radiant intensity, $I$, at wavelength $\lambda$ per unit wavelength interval. The SI unit is $\mathrm{W} \mathrm{m}^{-1} \mathrm{sr}^{-1}$, but a commonly used unit is $\mathrm{W} \mathrm{nm}{ }^{-1} \mathrm{sr}^{-1}$.

\section{SPECTRAL RADIANT POWER $\left(P_{\lambda}\right)$}

The radiant power at wavelength $\lambda$ per unit wavelength interval. The SI unit is $\mathrm{W} \mathrm{m}^{-1}$, but a commonly used unit is $\mathrm{W} \mathrm{nm}{ }^{-1}$.

\section{SPONTANEOUS EMISSION}

That mode of emission that occurs even in the absence of a perturbing external electromagnetic field. The transition between states, $\mathrm{n}$ and $\mathrm{m}$, is governed by the Einstein coefficient of spontaneous emission, $A_{\mathrm{nm}}$.

\section{SUBSTANCE-ASSISTED PHOTOREACTIONS}

See catalyzed photolysis.

\section{SUBSTANCE-CATALYZED PHOTOREACTIONS}

See catalyzed photolysis.

\section{SURFACE}

The external monolayer of a solid particle consisting of the regular array of surface atoms (or ions) and intrinsic and extrinsic surface defects of various types.

\section{SURFACE ACTIVE SITES}

Surface regular or defect sites which can initiate the chemical transformation of reagents.

SURFACE CONCENTRATION OF PHOTOCARRIERS $\left(n_{\mathrm{S}}\right)$

The number of photocarriers per unit volume in the spatial region of the surface of an irradiated solid (photocatalyst) particle. The quantity $n_{\mathrm{S}}$ is determined by the rate of carrier generation within the depth of penetration, by the lifetimes of free photocarriers, by the rate of surface recombination, by the diffusion length of carriers, by the electric field in the subsurface area, and by the characteristic particle size. 
The $n_{\mathrm{S}}$ quantity is often used in certain theoretical models of photocatalytic reactions. [units: $\mathrm{m}^{-3}$ or $\left.\mathrm{mol} \mathrm{m}{ }^{-3}\right]$.

\section{SURFACE RECOMBINATION}

The recombination of carriers at the solid surface. In theoretical models of heterogeneous photocatalysis, the photocarriers exchange between the solid and the reactants yielding products that can be considered as a specific type of surface recombination.

See rate of surface recombination.

\section{SURFACE STATES}

Energy levels arising from orbitals localized on atoms of the semiconductor lattice near a surface. They also refer to electronic energy levels of various types generated in the energy spectrum of the solid, particularly within the forbidden energy gap of semiconductors and insulators. They originate from a regular surface and from surface defects, including adsorbed species.

Note that surface states have a big effect on the electronic properties of any junction made with a semiconductor surface.

\section{THERMAL BLEACHING}

In photochemistry this term refers to the loss of absorption or emission intensity as a result of absorption of heat by the substrate. In semiconductor photochemistry and photophysics, it refers to the loss of color (loss of absorption) by the color centers as a result of heating the solid semiconductor or insulator.

\section{THERMOEMISSION}

A thermally induced transformation of a molecular structure or of a system (e.g., of a solution), thermally reversible, that produces a spectral change, typically but not necessarily of visible color.

\section{THERMOIONIZATION}

The release of a charge carrier from energy levels in a substrate resulting from absorption of heat by the substrate (molecule, semiconductor or insulator).

\section{THERMOLUMINESCENCE}

Luminescence arising from a reaction between species trapped in a rigid matrix and released as a result of an increase in temperature.

See luminescence.

\section{THERMOPHOTOCATALYSIS}

A (photo)catalytic phenomenon that occurs in circumstances when the system is also heated by light. In case of light with a high photon flux, it is possible that not only photochemical (quantum) but also thermochemical processes would proceed as a result of the system being heated through absorption of light energy. This often occurs upon exposure of a heterogeneous (photo)catalyst to pulsed IR-light or pulsed laser emission.

\section{TIME-RESOLVED MICROWAVE CONDUCTIVITY (TRMC)}

Technique which allows the quantitative and qualitative detection of radiation-induced charge separation by time-resolved measurement of the changes in microwave absorption resulting from the production and decay of charged and dipolar (molecular) entities.

\section{TRANSMITTANCE (T)}

The ratio of the transmitted spectral radiant power $\left(P_{\lambda}\right)$ to that incident on the sample $\left(P_{o}{ }^{\lambda}\right)$ :

$$
T=\frac{P_{\lambda}}{P_{\lambda}^{o}}
$$

Internal transmittance refers to energy loss by absorption, whereas the total transmittance is that due to absorption, reflection, scatter, etc....

It is also the fraction of light (photons) transmitted by a system; $T$ is the term in the sum: $\mathrm{A}+T+R=1$, where $\mathrm{A}$ is the absorptance and $R$ is the reflectance.

See also absorbance, attenuance, Beer-Lambert law.

\section{TRIBOLUMINESCENCE}

Luminescence resulting from the rubbing together of the surface of certain solids. For example, it can be produced when solids are crushed.

\section{TUNNELING}

The passage of a particle through a potential-energy barrier the height of which is larger than the energy of that particle. This effect is important for some processes involving the transfer of electrons and light atoms, particularly $\mathrm{H}$ atoms.

\section{TURNOVER FREQUENCY (TOF)}

In spite of the complexities noted earlier (see above), the turnover frequency TOF can be described as the number of molecules photo-converted per unit time (SI unit: molecules $\mathrm{s}^{-1}$ ). Note that the number of catalytic sites on the surface of the catalyst (in heterogeneous photocatalysis) or the number of photocatalyst molecules (in homogeneous photocatalysis) is not required here.

TOF is useful in comparing different photocatalysts. The inverse of TOF, namely (TOF) ${ }^{-1}$, defines the characteristic time limit of a given photocatalyzed process regardless of the number of photocatalytic active centers (molecules, radicals, particles) in the system.

\section{TURNOVER NUMBER (TON)}

The ratio of the number of photoinduced transformations for a given period of time to the number of photocatalytic sites (or centers, in heterogeneous photocatalysis) or to the number of photocatalyst molecules 
in homogeneous photocatalysis. TON is a dimensionless quantity.

\section{Note}

(1) To determine the turnover number TON for a heterogeneous photoreaction, the number of photocatalytic centers (or sites) must be known. These are equivalent to the "photocatalyst molecules" in homogeneous photocatalysis. Regrettably, however, the number of such catalytic centers is unknown in a heterogeneous system involving particles of solid photocatalysts. Nonetheless, it is possible to assess the number of potential photocatalytic centers for any given system. Using the total number of regular surface sites (surface lattice ions, or atoms: for example, suppose that $\mathrm{N}$ is determined to be $\sim 10^{15} \mathrm{~cm}^{-2}$ and $\mathrm{S}$ [also in $\left.\mathrm{cm}^{2}\right]$ is the total surface area of the solid photocatalyst, then $N \times S=N_{\mathrm{T}}$ gives the total number of such potential active sites $N_{\mathrm{T}}$ ) it is possible to define a lower limit of the TON knowing the magnitude of $N_{\mathrm{T}}$. This turnover quantity is sometimes referred to by some workers as the "areal turnover number"-however, it is more appropriate to refer to it as the "lower limiting value of TON".

Also note that when TON $>1$, the (photo)catalytic character of the reaction or process is confirmed, even if one uses the lower limit of TON.

(2) It must be emphasized that it is the number of photocatalytic centers (sites) in the ground (not active) state that must be used in determining TON in heterogeneous photocatalysis.

In homogeneous photocatalysis, TON is the ratio of the number of photoinduced tranformations to the number of photocatalyst molecules in their ground (not electronically excited) state.

\section{TURNOVER RATE (TOR)}

The ratio of the rate of photoinduced transformations of molecules $(\mathrm{dN} / \mathrm{d} t)$ to the number of photocatalytic active centers in a system (in heterogeneous photocatalysis) or to the number of photocatalyst molecules (in homogeneous photocatalysis), $N_{C}$.

$$
\text { TOR }=\frac{\mathrm{d} N / \mathrm{d} t}{N_{C}} \quad\left(\text { SI units: molecules site }{ }^{-1} \mathrm{~s}^{-1}\right)
$$

The number of photocatalytic active centers in a heterogeneous system is given by $N_{C}=n_{C} S$ where $n_{C}$ is the surface concentration of photocatalytic active centers [units of $\mathrm{cm}^{-2}$ or $\mathrm{m}^{-2}$ ], and $S$ is the full area of the uniformly irradiated surface in the system.
In homogeneous photocatalysis, the turnover rate is given by,

$$
\text { TOR }=\frac{\mathrm{d} C / \mathrm{d} t}{C_{m}} \quad\left(\text { SI units: } \mathrm{s}^{-1}\right)
$$

where $C$ is the volume concentration of reactants consumed (or products produced) and $C_{m}$ is the volume concentration of the photocatalyst molecules.

\section{UV DOSE}

A dose of ultraviolet (UV) radiation.

\section{UV STABILIZER}

A substance added to a sample to prevent photodeterioraiton by ultraviolet (UV) light.

See photochemical reaction.

\section{VALENCE BAND}

The highest energy continuum of energy levels in a semiconductor (or insulator) that is fully occupied by electrons at $0 \mathrm{~K}$.

See bandgap, conduction band, Fermi level.

\section{VIBRATIONAL REDISTRIBUTION}

Intra-entity redistribution of energy among the vibrational modes usually giving a statistical distribution of their populations, characterized by the "vibrational temperature". For large entities, the process does not require collisions.

\section{VIBRATIONAL RELAXATION}

The loss of vibrational excitation energy by an entity through energy transfer to the environment caused by collisions. The entity relaxes into vibrational equilibrium with its environment.

\section{WAVELENGTH $(\lambda)$}

The distance between two corresponding points on adjacent waves measured along the line of propagation of the electromagnetic radiation. The wavelength depends on the medium in which the wave propagates.

\section{WAVENUMBER $\left(v^{\prime}\right)$}

The reciprocal of the wavelength $\lambda$ or the number of waves per unit length of electromagnetic radiation along the direction of propagation. The SI unit is $\mathrm{m}^{-1}$, but a commonly used unit is $\mathrm{cm}^{-1}$.

\section{ACKNOWLEDGMENTS}

We thank the Natural Sciences and Engineering Research Council of Canada for their support of our work in photochemistry and photocatalysis through the years. 


\section{SYMBOLS}

Parameter

Absorbance

Absorptance

Absorption coefficient (decadic)

(Napierian)

Absorption Cross-Section

Attenuance

Avogadro's number

Concentration of defects or centers

Concentration of substrate

Depth of penetration (of light)

Efficiency (of a step)

(Energy) Fluence rate

Fermi level

Fluence

Frequency (linear)

$$
\text { (angular) }
$$

Interparticle electron transfer

Irradiance

Lifetime

Lifetime of an active state of a photo catalytic (or photoadsorption) center

Mass of photocatalyst

Maximal photoadsorption capacity of an adsorbent

Maximal number of molecules photoadsorbed

Molar (decadic) absorption coefficient,

Molar (Napierian) absorption coefficient

Number of molecules adsorbed after termination of irradiation

Number of photoadsorbed molecules during the same time of irradiation

Number of surface sites

Optical pathlength

Photoadsorption capacity (of an adsorbent)

Photoadsorption efficiency

Photochemical capacity of a heterogeneous photocatalyst (areal)

Photochemical capacity of a heterogeneous photocatalyst (volumetric)

Photodesorption cross-section

Photodesorption decay time

Photodesorption quantum yield

Photoinduced chesorluminescence

Photon excitance

Photon exposure

Photon flow

Photon fluence

Photon fluence rate

Photonic efficiency

Photon irradiance

Photon radiance $\left.{ }^{5}\right)$

Post-adsorption memory coefficient

Quantum efficiency

Quantum yield

\begin{tabular}{|c|c|}
\hline Symbol & Units \\
\hline \multicolumn{2}{|l|}{$A$} \\
\hline \multicolumn{2}{|l|}{ A } \\
\hline$a$ & $\mathrm{~m}^{-1}$, or $\mathrm{cm}^{-1}$ \\
\hline$\alpha$ & $\mathrm{m}^{-1}$, or $\mathrm{cm}^{-1}$ \\
\hline$\sigma$ & $\mathrm{m}^{2}$, or $\mathrm{cm}^{2}$ \\
\hline \multicolumn{2}{|l|}{$D$} \\
\hline \multicolumn{2}{|l|}{$N_{A}$} \\
\hline$n$ & $\mathrm{~cm}^{-3}$ \\
\hline$c$ & $\mathrm{~mol} \mathrm{dm}^{-3}$, or $\mathrm{M}$ \\
\hline $1 / a($ or $1 / \alpha)$ & $\mathrm{m}$, or $\mathrm{cm}$ \\
\hline \multicolumn{2}{|l|}{$\eta$} \\
\hline$E_{0}$ & $\mathrm{~W} \mathrm{~m} \mathrm{~m}^{-2}$ \\
\hline \multicolumn{2}{|l|}{$E_{\mathrm{F}}$} \\
\hline$H_{0}$ & $\mathrm{~J} \mathrm{~m}^{-2}$ \\
\hline$v$ & $\mathrm{~s}^{-1}$ \\
\hline$\omega$ & $\operatorname{rad~s} s^{-1}$ \\
\hline \multicolumn{2}{|l|}{ IPET } \\
\hline$E$ & $\mathrm{~W} \mathrm{~m} \mathrm{~m}^{-2}$ \\
\hline$\tau$ & S \\
\hline$T_{\mathrm{C}}$ & $\mathrm{s}$ \\
\hline$m$ & $\mathrm{~g}$ \\
\hline \multicolumn{2}{|l|}{$\theta_{\mathrm{m}}$} \\
\hline \multicolumn{2}{|r|}{ 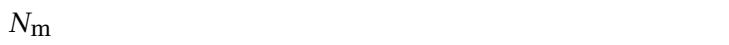 } \\
\hline$\varepsilon$ & $\mathrm{dm}^{-3} \mathrm{~mol}^{-1} \mathrm{~cm}^{-1}$, or $\mathrm{M}^{-1} \mathrm{~cm}^{-1}$ \\
\hline$\kappa$ & $\mathrm{dm}^{3} \mathrm{~mol}^{-1} \mathrm{~cm}^{-1}$, or $\mathrm{M}^{-1} \mathrm{~cm}^{-1}$ \\
\hline
\end{tabular}

$N_{\text {post }}(t)$

$N_{\text {phot }}(t)$

$N_{\text {ss }}$

$\mathrm{cm}^{-2}$

$l$

$\theta$

$(\mathrm{PAE})_{\lambda}$

(APC)

(VPC)

$\sigma_{\text {phd }}$

$\tau_{\text {phd }}$

$\Phi_{\text {des }}$

PhICL

$M_{\mathrm{P}}$

$H_{\mathrm{P}}$

$\rho$

$H_{\mathrm{P}}{ }^{0}$

$E_{\mathrm{P}}{ }^{0}$

$\xi$

$E_{\mathrm{P}}$

$L_{\mathrm{P}}$

K

$\eta$

$\Phi$ $\mathrm{m}$, or $\mathrm{cm}$

einstein $\mathrm{m}^{-2} \mathrm{~s}^{-1}$

einstein $\mathrm{m}^{-2} \mathrm{~s}^{-1}$ $\mathrm{m}^{2}$, or $\mathrm{cm}^{2}$

$\mathrm{s}$

$\mathrm{mol} \mathrm{m}{ }^{-1} \mathrm{~s}^{-1}$

$\mathrm{m}^{-2}$

$\mathrm{mol} \mathrm{s}^{-1}$

quanta $\mathrm{m}^{-2}$

mol $\mathrm{m}^{-2} \mathrm{~s}^{-1}$, or $\mathrm{m}^{-2} \mathrm{~s}^{-1}$

$\mathrm{mol} \mathrm{m} \mathrm{m}^{-2} \mathrm{~s}^{-1}$, or $\mathrm{m}^{-2} \mathrm{~s}^{-1}$

$\mathrm{m}^{-2} \mathrm{~s}^{-1}$, or $\mathrm{m}^{-2} \mathrm{~s}^{-1} \mathrm{sr}^{-1}$, or $\mathrm{mol} \mathrm{m}^{-2} \mathrm{~s}^{-1} \mathrm{sr}^{-1}$ 


\begin{tabular}{|c|c|c|}
\hline Radiance & $L$ & $\mathrm{~W} \mathrm{~m} \mathrm{~m}^{-2}$, or $\mathrm{W} \mathrm{m} \mathrm{m}^{-2} \mathrm{sr}^{-1}$ \\
\hline Radiant Energy & $Q$ & $\mathrm{~J}$ \\
\hline Radiant energy flux (or radiant power) & $P$ & $\mathrm{~J} \mathrm{~s}^{-1}$ \\
\hline Radiant excitance & $M$ & $\mathrm{~W} \mathrm{~m}^{-2}$ \\
\hline Radiant exposure & $H$ & $\mathrm{~W} \mathrm{\textrm {m } ^ { - 2 }} \mathrm{s}$, or $\mathrm{J} \mathrm{m}^{-2}$ \\
\hline Radiant intensity & $I$ & $\mathrm{~W} \mathrm{sr}{ }^{-1}$ \\
\hline Radiant power & $P$ & $\mathrm{~J} \mathrm{~s}^{-1}(=\mathrm{W})$ \\
\hline Rate of surface recombination & $s$ & $\mathrm{~m} \mathrm{~s}^{-1}$ \\
\hline Rate of (photo)generation (of carriers) & g & $\mathrm{mol} \mathrm{m}^{-3} \mathrm{~s}^{-1}$, or $\mathrm{m}^{-3} \mathrm{~s}^{-1}$ \\
\hline Reflectance & $R$ & \\
\hline Relative photonic efficiency & $\xi_{\text {rel }}$ & \\
\hline Specific surface area & $\sigma$ & $\mathrm{cm}^{2} \mathrm{~g}^{-1}$ \\
\hline Spectral Irradiance & $E_{\lambda}$ & $\mathrm{W} \mathrm{m}^{-3}$, or $\mathrm{W} \mathrm{m} \mathrm{m}^{-2} \mathrm{~nm}^{-1}$ \\
\hline Spectral photon excitance $\left({ }^{5}\right)$ & $M_{p \lambda}$ & $\begin{array}{l}\mathrm{s}^{-1} \mathrm{~m}^{-3} \text {, or s } \mathrm{s}^{-1} \mathrm{~m}^{-2} \mathrm{~nm}^{-1} \text {, or } \mathrm{mol} \mathrm{s} \mathrm{s}^{-1} \mathrm{~m}^{-1} \text {, } \\
\text { or } \mathrm{mol} \mathrm{s}^{-1} \mathrm{~m}^{-2} \mathrm{~nm}^{-1}\end{array}$ \\
\hline Spectral photon flow $\left({ }^{5}\right)$ & $\rho_{\lambda}$ & $\begin{array}{l}\mathrm{s}^{-1} \mathrm{~m}^{-1} \text {, or } \mathrm{s}^{-1} \mathrm{~nm}^{-1}, \text { or } \mathrm{mol} \mathrm{m} \mathrm{m}^{-3} \mathrm{~s}^{-1} \\
\text { or } \mathrm{mol} \mathrm{m} \mathrm{mm}^{-2} \mathrm{~nm}^{-1} \mathrm{~s}^{-1}\end{array}$ \\
\hline Spectral photon flux (photon irradiance) $\left({ }^{5}\right)$ & $E_{p \lambda}$ & $\begin{array}{l}\mathrm{m}^{-3} \mathrm{~s}^{-1}, \text { or } \mathrm{m}^{-2} \mathrm{~nm}^{-1} \mathrm{~s}^{-1} \\
\mathrm{~mol} \mathrm{~m}^{-3} \mathrm{~s}^{-1}, \text { or } \mathrm{mol} \mathrm{m}^{-2} \mathrm{~nm}^{-1} \mathrm{~s}^{-1}\end{array}$ \\
\hline Spectral photon radiance $\left({ }^{5}\right)$ & $L_{p \lambda}$ & $\begin{array}{l}\mathrm{s}^{-1} \mathrm{~m}^{-3} \mathrm{sr}^{-1}, \text { or s }^{-1} \mathrm{~m}^{-2} \mathrm{sr}^{-1} \mathrm{~nm}^{-1}, \\
\text { or } \mathrm{mol} \mathrm{s}^{-1} \mathrm{~m}^{-3} \mathrm{sr}^{-1} \text {, or mol s} \mathrm{m}^{-1} \mathrm{~m}^{-2} \mathrm{sr}^{-1} \mathrm{~nm}^{-1}\end{array}$ \\
\hline Spectral radiance & $L_{\lambda}$ & $\mathrm{W} \mathrm{m}^{-3} \mathrm{sr}^{-1}$, or $\mathrm{W} \mathrm{m} \mathrm{m}^{-2} \mathrm{sr}^{-1} \mathrm{~nm}^{-1}$ \\
\hline Spectral radiant excitance & $M_{\lambda}$ & $\mathrm{W} \mathrm{m} \mathrm{m}^{-3}$, or $\mathrm{W} \mathrm{m}^{-2} \mathrm{~nm}^{-1}$ \\
\hline Spectral radiant intensity & $I_{\lambda}$ & $\mathrm{W} \mathrm{m}{ }^{-1} \mathrm{sr}^{-1}$, or $\mathrm{W} \mathrm{nm} m^{-1} \mathrm{sr}^{-1}$ \\
\hline Spectral radiant power & $P_{\lambda}$ & $\mathrm{W} \mathrm{m}^{-1}$, or $\mathrm{W} \mathrm{nm}^{-1}$ \\
\hline Surface concentration of photocarriers & $n_{\mathrm{S}}$ & $\mathrm{m}^{-3}$, or $\mathrm{mol} \mathrm{m}^{-3}$ \\
\hline Time-resolved microwave conductivity & TRMC & \\
\hline Transmittance & $T$ & \\
\hline Turnover frequency & TOF & molecules s ${ }^{-1}$ \\
\hline Turnover number & TON & \\
\hline Turnover rate (in heterogeneous photocatalysis) & TOR & molecules site ${ }^{-1} \mathrm{~s}^{-1}$ \\
\hline (in homogeneous photocatalysis) & TOR & $\mathrm{s}^{-1}$ \\
\hline Wavelength & $\lambda$ & $\mathrm{nm}$, or $\mathrm{A}$ \\
\hline Wavenumber & $v^{\prime}$ & $\mathrm{cm}^{-1}$ \\
\hline
\end{tabular}

$\left({ }^{5}\right)$ See text.

\section{References}

[1] K. I. Zamaraev, Studies in Surface Science and Catalysis, J. W. Hightower, W. N. Delgass, E. Iglesia and A. T. Bell (eds.), Vol. 101, part A, Elsevier, Amsterdam, 1996, p. 35.

[2] V. N. Parmon, Fotokataliticheskoe Preobrazovanie Solnechnoj Energii (Photocatalytic Conversion of Solar Energy), V. N. Parmon and K. I. Zamaraev (eds.), Nauka, Novosibirsk, 1991, p. 7 (in Russian).

[3] O. V. Gerasimov and V. N. Parmon, Russ. Chem. Rev. 61 (1992), 293.

[4] V. N. Parmon, Catal. Today 39 (1997), 137.

[5] S. E. Braslavsky and K. N. Houk, Pure \& Appl. Chem. 60 (1988), 1055.

[6] J. W. Verhoeven, Pure \& Appl. Chem. 68 (1996), 2223.

[7] Compendium of Chemical Terminology, IUPAC Recommendations, A. D. McNaught and A. Wilkinson (eds.), London, Blackwell, 1977.
[8] N. Serpone and A. Salinaro, Pure \& Appl. Chem. 71 (1999), 303.

[9] A. Salinaro, A. V. Emeline, J. Zhao, H. Hidaka, V. K. Ryabchuk, and N. Serpone, Pure \& Appl. Chem. 71 (1999), 321.

[10] N. Serpone, A. Salinaro, A. V. Emeline, and V. K. Ryabchuck, J. Photochem. Photobiol. A: Chem. 130 (2000), 83.

[11] H. Kisch, Photocatalysis - Fundamentals and Applications, N. Serpone and E. Pelizzetti (eds.), WileyInterscience, New York, 1989, Chapt. 1.

[12] Glossary of Terms in Photochemistry, EPA Newsletter 25 (1985), 13.

[13] F. Chanon and M. Chanon, Photocatalysis - Fundamentals and Applications, N. Serpone and E. Pelizzetti (eds.), Wiley-Interscience, New York, 1989, Chapt. 15.

[14] S. J. Teichner and M. Formenti, Photoelectrochemistry, Photocatalysis and Photoreactors, 
M. Schiavello (ed.), Reidel, Dordrecht, 1985, pp. 457-489.

[15] R. G. Salomon, Tetrahedron 39 (1983), 485.

[16] C. Kutal, Adv. Chem. Ser. 238 (1993), 1.

[17] C. Kutal, Coord. Chem. Rev. 64 (1985), 191.

[18] H. Hennig, D. Rehorek, and R. D. Archer, Coord. Chem. Rev. 61 (1985), 1.

[19] N. Serpone, E. Pelizzetti and H. Hidaka, Photochemical and Photoelectrochemical Conversion and Storage of Solar Energy, Z. W. Tian and Y. Cao (eds.), International Academic Publishers, Beijing, 1993, p. 33.

[20] G. G. Wubbels, Acc. Chem. Res. 16 (1983), 285

[21] M. J. Mirbach, EPA Newsletter 20 (1984), 16.

[22] L. Moggi, A. Juris, D. Sandrini, and M. F. Manfrin, Rev. Chem. Intermed. 5 (1981), 107.

[23] L. P. Childs and D. F. Ollis, J. Catal. 66 (1980), 383.

[24] K. J. Laidler, Chemical Kinetics, 3rd edition, HarperCollins Publishers, New York, 1987, Chapt. 10.

[25] J. B. Butt and E. E. Petersen, Activation, Deactivation and Poisoning of Catalysts, Academic Press, San Diego, CA, 1988, Chapt. 4.

[26] B. C. Gates, Catalytic Chemistry, Wiley, New York, 1992, Chapt. 3 and 6.

[27] Manual of Symbols and Terminology for Physicochemical Quantities and Units (Part II: heterogeneous catalysis), Adv. Catal. 26 (1977), 351 and 372.

[28] M. Boudart and G. Djega-Mariadassou, Kinetics of Heterogeneous Catalytic Reactions, Princeton University Press, 1984, pp. 6-8.

[29] M. Boudart, Chem. Rev. 95 (1995), 661.

[30] N. A. Mohd-Zabidi, D. Tapp, and T. F. Thomas, J. Phys. Chem. 99 (1995), 14733.

[31] M. A. Fox, personal communication to N. Serpone, July 1995.

[32] G. Somorjai, Photocatalysis - Fundamentals and Applications, N. Serpone and E. Pelizzetti (eds.), Wiley-Interscience, New York, 1989, Chapt. 9.

[33] (a) L. L. Basov, G. N. Kuzmin, I. M. Prudnikov, and Yu. P. Solonitzyn, Uspekhi Photoniki., Th. I. Vilesov (ed.), Vol. 6, LGU (Leningrad State University), 1977, p 82.

(b) A. V. Emeline and V. K. Ryabchuk, Russ. J. Phys. Chem. 71 (1997), 2085.

(c) S. V. Kurganov and Yu. M. Artem'ev, Vestnik LGU, Iss. 4: Physics, Chemistry 4 (1988), 9.

[34] M. Schiavello, V. Augugliaro, and L. Palmisano, J. Catal. 127 (1991), 332.
[35] A. V. Emeline, V. K. Ryabchuck, and N. Serpone, J. Phys. Chem. B 103 (1999), 1316.

[36] Th. Th. Volkenstein, Electronic Processes on the Surface of Semiconductors during Chemisorption, Nauka, Moscow, 1987.

[37] K. I. Zamaraev and V. N. Parmon, Energy Resources through Photochemistry and Catalysis, M. Grätzel (ed.), Academic Press, N.Y., 1983, p. 127.

[38] J. S. Connolly (ed.), Photochemical Conversion and Storage of Solar Energy, Academic Press., N.Y., 1982.

[39] L. Palmisano, V. Augugliaro, R. Campostrini, M. Schiavello, J. Catal. 143 (1993), 149.

[40] V. Augugliaro, L. Palmisano, and M. Schiavello, AICh. J. 37 (1991), 1096.

[41] M. I. Cabrera, O. M. Alfano, A. E. Cassano, Ind. Eng. Chem. Res. 33 (1994), 3031.

[42] A. M. Braun, M.-T. Maurette, and E. Oliveros, Photochemical Technology, Wiley, New York, 1991, Chapt. 2.

[43] D. W. Bahnemann, D. Bockelmann, R. Goslich, Sol. Energy Mater. 24 (1991), 564.

[44] P. Pichat, Photochemistry, Photocatalysis, and Photoreactors, M. Schiavello (ed.), Reidel, Dordrecht, Holland, 1985, pp. 425-455.

[45] N. Serpone, E. Pelizzetti, and H. Hidaka, Photochemical and Photoelectrochemical Storage of Solar Energy, Z. W. Tian and Y. Cao (eds.), International Academic Publishers, Beijing, China, 1993, pp. 33-73.

[46] N. Serpone, R. Terzian, D. Lawless, P. Kennepohl, G. Sauve, J. Photochem. Photobiol. A: Chem. 73 (1993), 11.

[47] N. Serpone, G. Sauve, R. Koch, H. Tahiri, P. Pichat, P. Piccinini, E. Pelizzetti, and H. Hidaka, J. Photochem. Photobiol. A: Chem. 94 (1996), 191.

[48] V. N. Parmon, Yu. I. Aristov, Radiation Catalytic Processes in Dispersed Media, V. N. Parmon (ed.), Novosibirsk, Nauka, 1992, p. 6 (in Russian).

[49] K. S. W. Sing, D. H. Everett, R. A. W. Haul, L. Moscou, R. A. Pierotti, J. Rouquerol, T. Siemieniewska, Pure \& Appl. Chem. 57 (1985), 603.

[50] R. J. Brandi, O. M. Alfano, A. E. Cassano, Env. Sci. Technol. 34 (2000), 2623.

[51] R. J. Brandi, O. M. Alfano, and A. E. Cassano, Env. Sci. Technol. 34 (2000), 2631.

[52] O. M. Alfano, M. I. Cabrera, and A. E. Cassano. J. Catal 172 (1997), 370. 


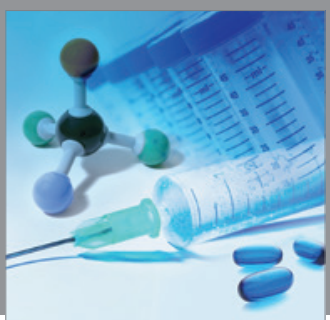

International Journal of

Medicinal Chemistry

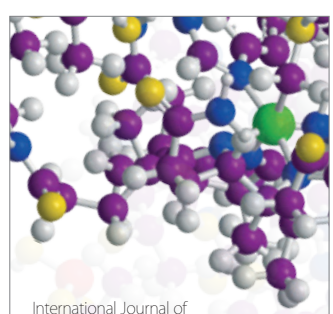

Carbohydrate Chemistry

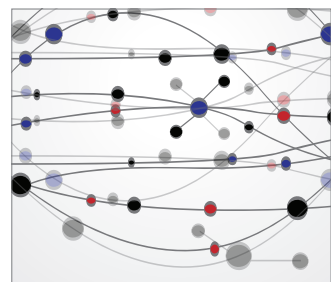

The Scientific World Journal
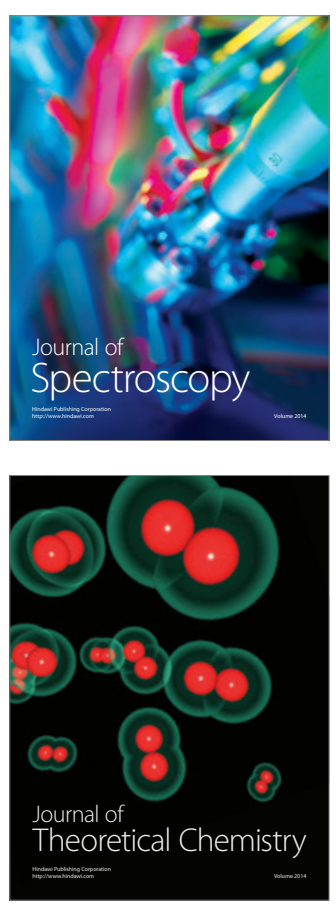
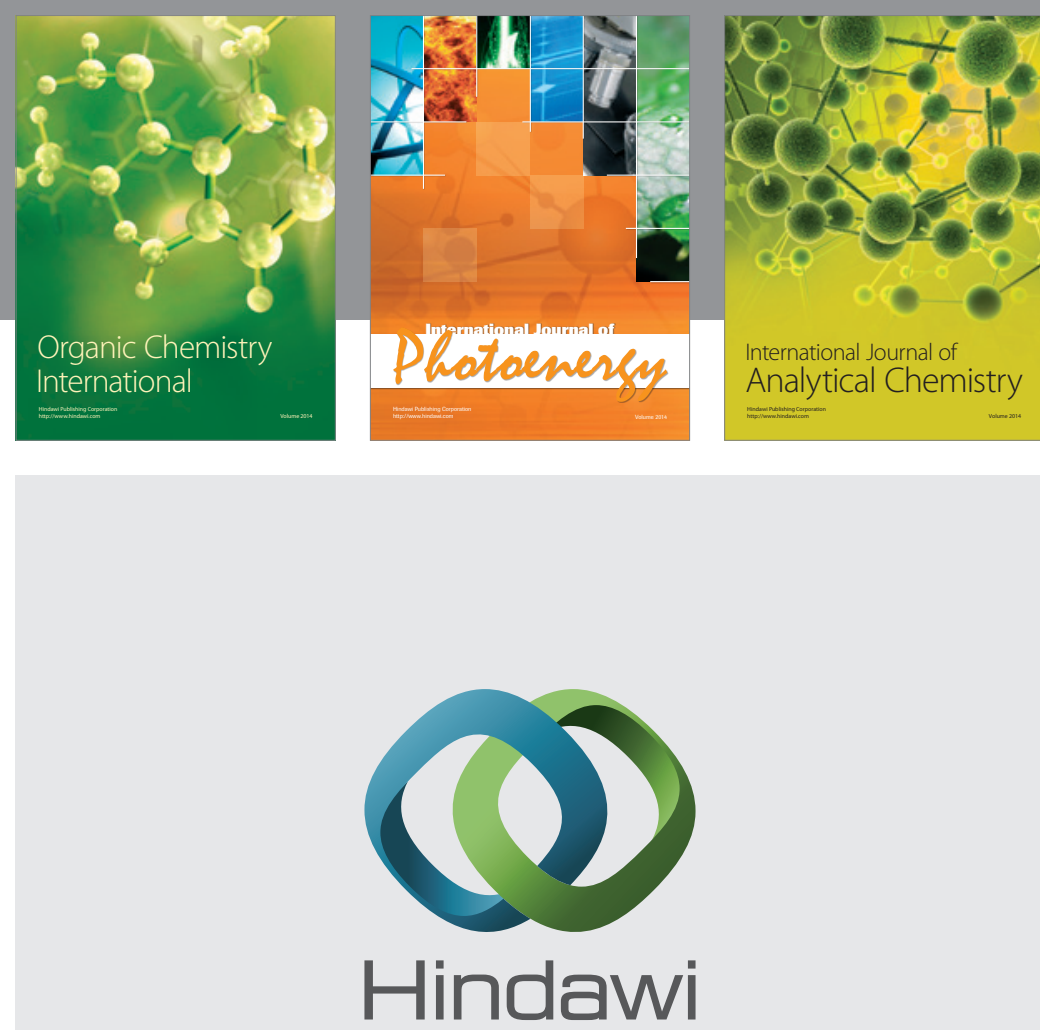

Submit your manuscripts at

http://www.hindawi.com
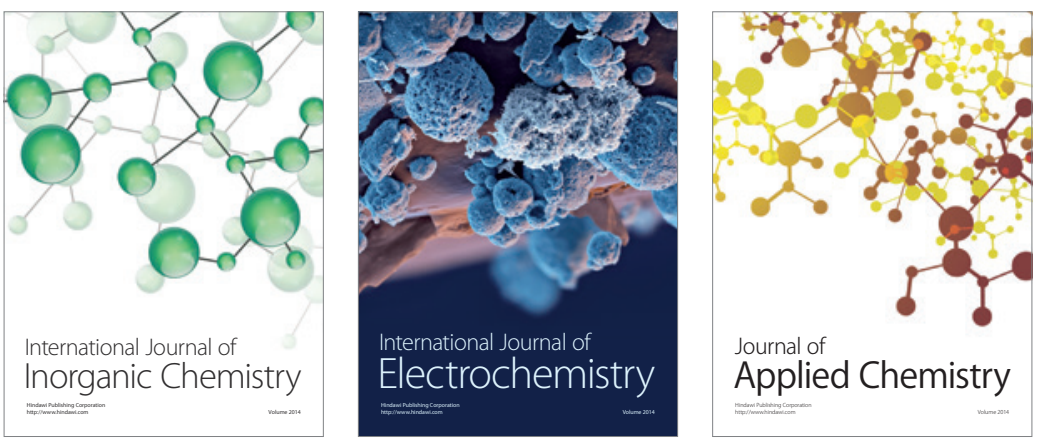

Journal of

Applied Chemistry
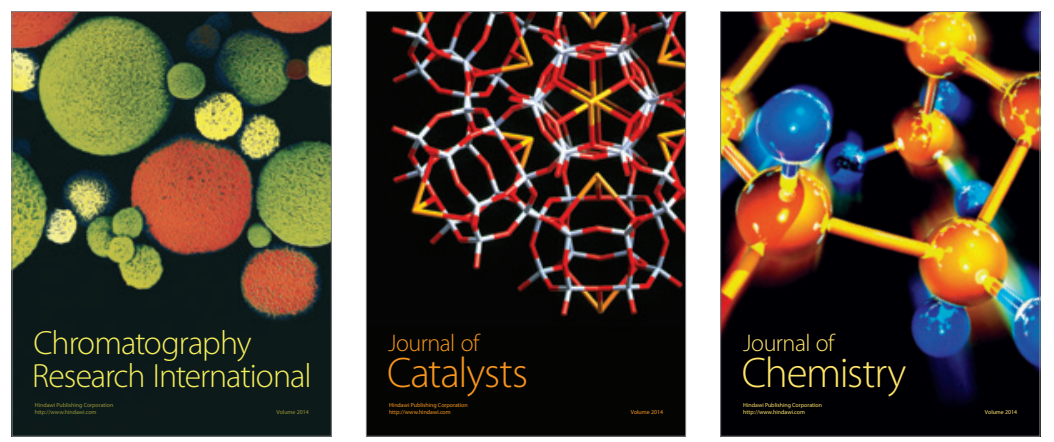
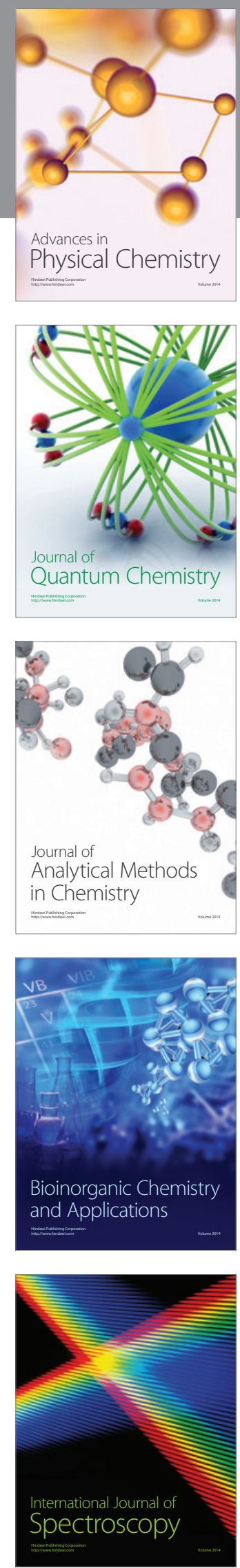